Noname manuscript No.

(will be inserted by the editor)

\title{
Slow solar wind: observations and modeling
}

\author{
L. Abbo · L. Ofman - S.K. Antiochos . \\ V.H. Hansteen · L. Harra · Y-K. Ko \\ G. Lapenta · B. Li · P. Riley · L. Strachan . \\ R. von Steiger · Y.-M. Wang
}

Received: date / Accepted: date

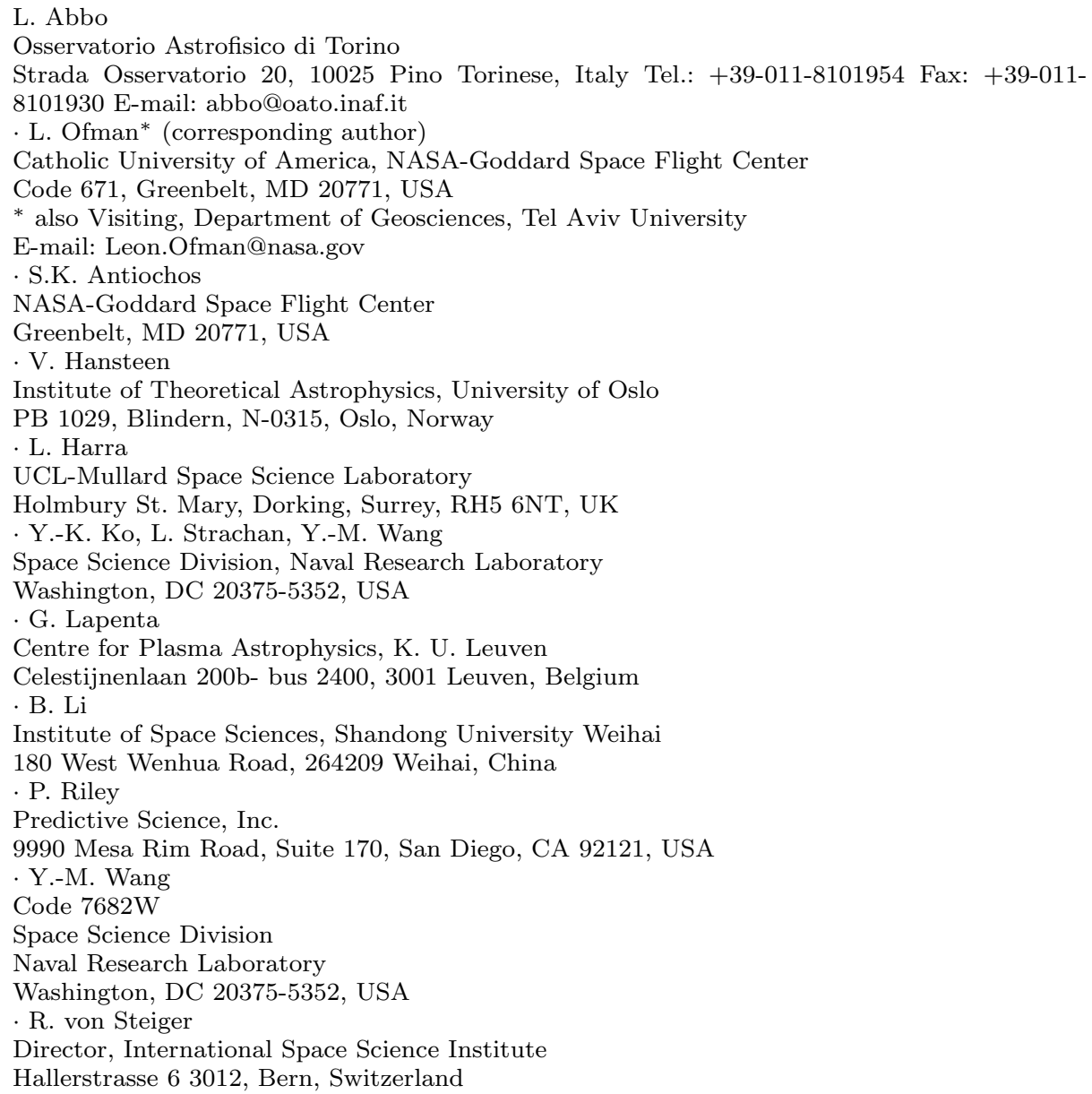


Abstract While it is certain that the fast solar wind originates from coronal holes, where and how the slow solar wind (SSW) is formed remains an outstanding question in solar physics even in the post-SOHO era. The quest for the SSW origin forms a major objective for the planned future missions such as the Solar Orbiter and Solar Probe Plus. Nonetheless, results from spacecraft data, combined with theoretical modeling, have helped to investigate many aspects of the SSW. Fundamental physical properties of the coronal plasma have been derived from spectroscopic and imaging remote-sensing data and in situ data, and these results have provided crucial insights for a deeper understanding of the origin and acceleration of the SSW. Advanced models of the SSW in coronal streamers and other structures have been developed using 3D MHD and multi-fluid equations.

However, the following questions remain open: What are the source regions and their contributions to the SSW? What is the role of the magnetic topology in the corona for the origin, acceleration and energy deposition of the SSW? What are the possible acceleration and heating mechanisms for the SSW? The aim of this review is to present insights on the SSW origin and formation gathered from the discussions at the International Space Science Institute (ISSI) by the Team entitled "Slow solar wind sources and acceleration mechanisms in the corona" held in Bern (Switzerland) in March 2014 and 2015.

Keywords Sun · Corona - Solar wind - Coronal streamers · MHD and kinetic models

\section{Contents}

1 Introduction . . . . . . . . . . . . . . . . . . . . . . . . . . . . 3

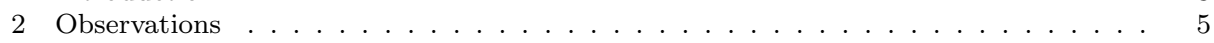

2.1 Spectroscopic data: from the lower to the extended corona . . . . . . . . . 5

2.1.1 Some results from Hinode/EIS - XRT instruments . . . . . . . . . . . . 5

2.1.2 Some results from SOHO/UVCS instrument . . . . . . . . . . . . . . 8

2.2 Imaging Data: some results from SOHO/LASCO and STEREO/COR Corona-

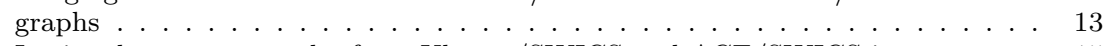

2.3 In-situ data: some results from Ulysses/SWICS and ACE/SWICS instruments . 15

2.3.1 Ulysses ........................... 17

2.3.2 ACE/SWICS ......................... 20

3 Numerical modeling . . . . . . . . . . . . . . . . . . . . . . . . . . . . . . . . . . 24

3.1 Connecting transition region and coronal models . . . . . . . . . . . . . . . . . 24

3.2 3D MHD single fluid models . . . . . . . . . . . . . . . . . . . . . 26

$3.32 \mathrm{D}$ and 3D MHD multi-fluid models . . . . . . . . . . . . . . . . . . . . . . . . 29

4 Which are the main SSW sources and their formation mechanisms? . . . . . . . . . . 35

4.1 Expansion factor model . . . . . . . . . . . . . . . . . . . . . 36

4.2 Interchange reconnection vs. expansion factor models . . . . . . . . . . . . . . 40

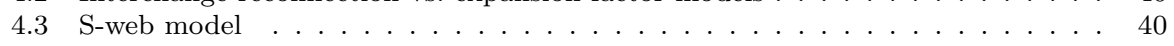

4.3.1 The origin of S-web arcs . . . . . . . . . . . . . . . . . . . . . . . . . . 42

4.4 Magnetic reconnection at the streamer cusp . . . . . . . . . . . . . . . . . . 44

4.5 Discussion ............................ . . . . 46

5 Which are the main physical mechanisms for the SSW acceleration? . . . . . . . . 48

6 Conclusions .................................... . . 49 


\section{Introduction}

The solar wind is a continuous stream of charged particles (mainly protons, electrons, and $\alpha$ particles, but also heavy ions) ejected from the Sun at an average mass loss rate of $(2 \sim 3) \times 10^{-14} \mathrm{M}_{\odot} /$ year. At solar minimum, the identification of two types of solar wind has been accepted for decades: fast wind originating from coronal holes and slow wind related to the streamer belt regions (e.g., McComas et al, 2008).

The present review focuses on the slow solar wind (SSW). The SSW $(<\mathbf{5 0 0}$ $\mathrm{km} \mathrm{s}^{-1}$ ) is in many ways different from the fast solar wind, such as higher proton density, lower proton temperature, higher electron temperature, larger temporal variability, higher heavy ion ionization states and higher first ionization potential (FIP) bias in elemental abundances (e.g., review by Schwenn, 2006; Geiss et al, 1995b; von Steiger et al, 2000, and references within). Such general properties of the SSW can be derived from ensemble studies that group the solar wind from multiple solar wind streams over a range of time periods.

The SSW tends to encompass the heliospheric current sheet (HCS), but it extends up to $30^{\circ}$ in heliocentric latitude (McComas et al, 2008; Wang et al, 2000). The HCS often is not located in the middle of the SSW region, but near a stream interface boundary (Burlaga et al, 2002) owing to the fast speed wind of the next stream catching up from behind (Pizzo, 1978). In some SSW streams, the location of the HCS is displaced from that of the magnetic sector boundary (as indicated by the direction of the suprathermal electron flux relative to the interplanetary magnetic field), supporting the occurrence of interchange reconnection at the streamer/coronal hole boundary (Crooker et al, 2004). A portion of the SSW is not associated with a HCS at the stream interface, indicating the presence of a pseudostreamer at its solar origin (Neugebauer et al, 2004; Crooker et al, 2012).

The in situ solar wind measurements in the heliosphere have found that some slow speed wind bears some signatures of the fast speed wind (e.g., Zhao et al, 2009; D'Amicis and Bruno, 2015). Thus, clearly speed is not the only indicator for the origin of solar wind. Since the ionic charge states and elemental abundances are largely fixed in the inner corona (generally below $10 R_{\odot}$ ), as opposed to density and temperature which change dynamically during the transit in the heliosphere, the heavy ion charge state and elemental compositions are most intimately related to the properties in the solar wind source region (see Sections 2 and 2.3). Therefore these measurements provide important information, along with remote-sensing observations, about the formation of the solar wind and the SSW sources.

From spectroscopic data, important observational results have been obtained in recent years (see, Section 2.1). In the low corona, persistent outflows at the edges of active regions have been found by the analysis of data from several instruments, and their contribution to the SSW has been investigated with magnetic 
field models of the large-scale coronal structures. Recent results link the outflows further to the compositional data (see, Section 2.1.1). From the ultraviolet (UV) spectroscopy of the extended corona, it has been found that a significant amount of SSW originates outside the streamer/coronal hole boundaries, where the wind velocity depends on the magnetic field topology of flux tubes. Moreover, some indications about the SSW outflow velocity profile and consequently the acceleration have been derived from UVCS observations (see, Section 2.1.2). White light coronagraphic observations provide critical evidence linking the solar-minimum streamer belt with the SSW: several small CMEs in the form of slow "streamer blowouts" were observed and they were considered as tracers of the SSW (see, Section 2.2). Some recent results based on UV and white light (WL) observations of pseudo-streamers have shown that these multi-polar structures can contribute to the SSW as well (see, Sections 2.1 and 2.2). Compositional data from in situ observations at $1 \mathrm{AU}$ and beyond provide clues on the origin of the SSW by relating the measurements to the coronal source regions (see, Section 2.3). It is evident that important parameters such as the temperatures and charge states of heavy ion species and in particular the oxygen ions and proton flux distributions are fundamental for characterizing the SSW (see Section 2.3.1) in addition to the widely used outflow velocity and variability features. Moreover, slow wind regions with similar speeds can exhibit different physical parameters, which implies also different source region properties (see, Section 2.3.2), providing a wealth of clues that have to be considered in understanding how the SSW is formed.

Observational evidences provides important constraints for the solar wind models (see, Section 3). The study of the solar wind and its global structure and evolution is strongly linked to the study of the transition region and the chromosphere, but modeling the transition region dynamics is quite complex (see Section 3.1). From the theoretical point of view, in the solar corona, the ejection of material may be caused by the loss of confinement due to pressure-driven instabilities as the heated plasma accumulates or due to current-driven instabilities (tearing, Kelvin-Helmholtz, and kink-type instabilities) in the sheared field of a streamer. On small scales compared to the typical global streamer structures, $2.5 \mathrm{D}$ (two spatial dimensions and three components of the velocity and magnetic field) magnetohydrodynamic (MHD) models can account for the plasmoid blowouts observed in helmet streamers: these are likely due to magnetic reconnection at the current sheet above the cusp and are accelerated by the combined effects of the tearing mode and Kelvin-Helmholtz instabilities (KHIs) triggered by the reconnection in the current sheet (see Section 4.4). The MHD approximation is appropriate for large-scale (compared to the gyroradius), low-frequency phenomena in magnetized plasmas such as the solar corona. Using the photospheric magnetic field as the primary driving boundary condition, single-fluid 3D MHD models can often reproduce the Sun's magnetic and emission properties on the global scale structures of the corona (see, Section 3.2). The global 3D MHD models solve typically the ideal MHD equations, so the reconnection is not physically modeled, but relies on numerical resistivity. Multi-fluid 2.5D and recently 3D models of the SSW have been developed to simulate streamers that contain heavy ions (in addition to protons and electrons). Nevertheless, only very few multi-fluid studies of the SSW have attempted to reproduce the streamer observations by considering heavy ions self-consistently (see Section 3.3). 
Our present review addresses the main SSW sources and their formation mechanisms based on leading observations and modeling approaches. Scenarios discussed in Section 4 include: expansion factor model (Section 4.1), interchange reconnection model (Section 4.2), the S-web model (Section 4.3), and magnetic reconnection at the cusp of the streamers (Section 4.4). In sources where closed and open magnetic fields are in contact with reverse polarity, the energization of the SSW may occur through reconnection. However, in open field sources reconnection cannot explain the formation of the SSW, and a turbulence/wave mechanism is apparently appropriate. Section 5 is dedicated to the discussion on the main physical mechanisms for the SSW acceleration. Finally, in Section 6 we outline the main conclusions and some suggestions for future observation planning in order to address the still open questions of SSW formation and sources.

\section{Observations}

Crucial results on the origin of the solar wind have been derived from both remote sensing and in situ observations. In this section we review a selection of important observational aspects arising from the analyses of data from several instruments, which provide constraints for the solar wind models. We start from the lower corona, going through the extended corona and finally to the in-situ measurements in the heliosphere.

2.1 Spectroscopic data: from the lower to the extended corona

\subsubsection{Some results from Hinode/EIS - XRT instruments}

In the last 7 years, there are several results from Hinode observations showing that active regions, and particularly the edges of active regions, are good candidates for SSW sources. They have shown persistent upflows at the edges of active regions. These had been seen in some examples pre-Hinode with CDS and SUMER instruments onboard SOHO (e.g., Brekke et al, 1997; Teriaca et al, 1999). Upflows were initially observed with the X-ray telescope (XRT) and the EUV Imaging Spectrometer (EIS) on Hinode by Sakao et al (2007). The X-ray images showed pulsing features moving upwards at the edges of the active regions in areas of relatively weak emission. This flowing plasma reached speeds of $100 \mathrm{~km}$ $\mathrm{s}^{-1}$. The EIS measurements showed that this region has persistent blue-shifted plasma (Fig. 1). It was estimated that this upflowing plasma could form around $25 \%$ of the SSW. This potential linkage to the solar wind triggered a series of papers that aimed at confirming or denying this possibility and at understanding more completely the physical attributes of these upflows.

Del Zanna (2008) showed that persistent blueshifts, stronger in the hotter lines (typically $5-20 \mathrm{~km} \mathrm{~s}^{-1}$ in Fe XII and $10-30 \mathrm{~km} \mathrm{~s}^{-1}$ in Fe XV), were present in areas of weak emission, in a sharp boundary between the low-lying 'hot' 3 MK loops and 


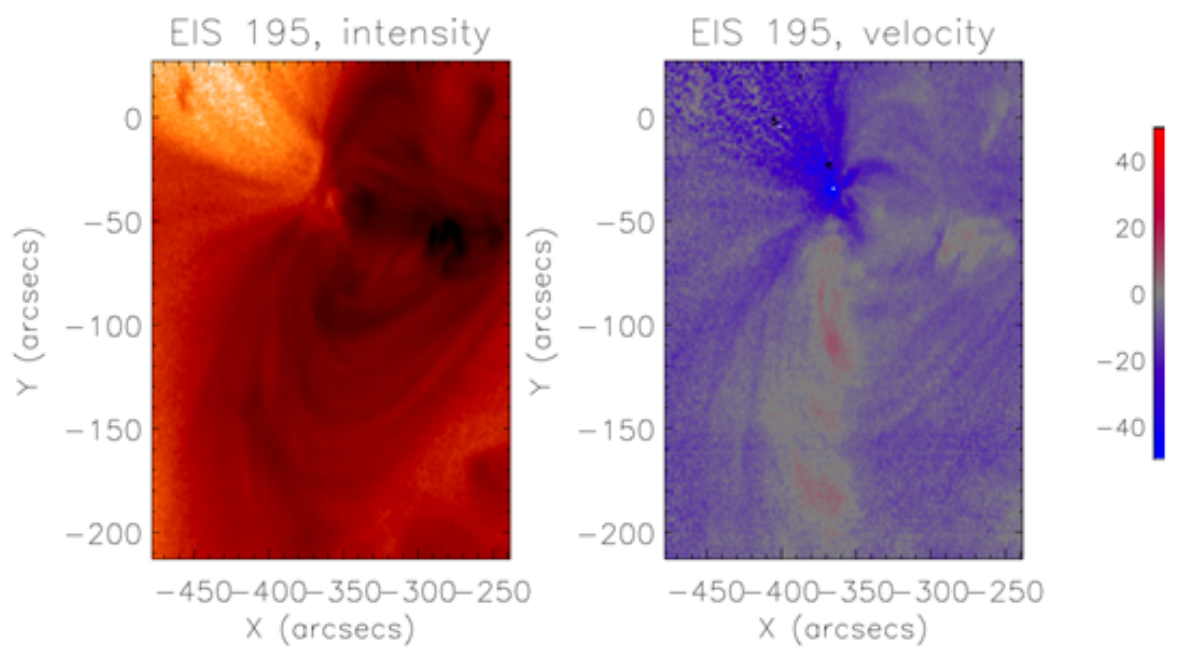

Fig. 1 EIS intensity image in Fe XII of an active region on the left. EIS Doppler velocity image on the right. From Harra et al (2008).

the higher 'cooler' 1 MK loops. The physical characteristics of the upflowing material were initially determined by Doschek et al (2008). They found temperatures of $1.3 \mathrm{MK}$ at low densities of $7 \times 10^{8} \mathrm{~cm}^{-3}$ in these upflow regions. The Doppler velocities and the excess line width measurements (non-thermal velocities) show a positive correlation. Hence a range of velocities exists in these regions. The line profiles are not always a single Gaussian profile, which is Doppler-shifted. UgarteUrra and Warren (2011) found that the enhancements in the blue wing of the emission line appear in timescales of 5 minutes - the process that creates the upflows is very dynamic. The upflowing features are coronal in nature being measured at temperatures of around 1MK (Warren et al, 2011). The emission below $1 \mathrm{MK}$ in the transition region shows up as red-shifted plasma. Brooks and Warren (2012) determined the chemical composition using Hinode EIS and found it to be consistent with the composition measured by ACE of the SSW. They have extended this work, analyzing together rasters of the full Sun. This took 48 hours to complete, and resulted in a full Sun map of the Doppler velocity and plasma composition providing a unique view of the whole Sun and contributions to the slow wind. The solar wind composition plasma was found to exist on regions that, from magnetic modeling, show open magnetic field lines. When these regions are summed up they provide a significant fraction of the mass loss rate of the solar wind (see Fig. 2 in Brooks et al, 2015). The plasma composition of the corona is key to understanding these sources but little is known about how active region composition varies with time. It was previously reported, based on Skylab results (Widing and Feldman, 2001), that the FIP bias (abundance ratio of a low-FIP element to a high-FIP element relative to their photospheric ratio) increases from photospheric to coronal values following the emergence of active regions. Baker et al (2015) further addressed this by studying an active region as it decays and determining the change in the FIP bias during this time. It was expected that the FIP bias would become more coronal-like as the active 

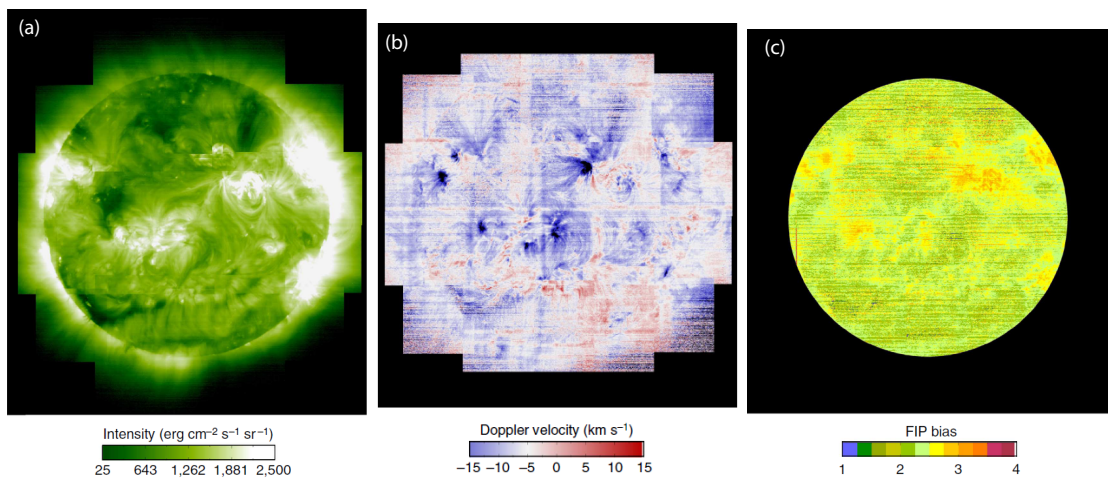

Fig. 2 (a) EIS image of the solar corona at 2 MK from Fe XIII $202.044 \AA$ spectral line intensity, (b) Doppler velocity map; blue areas highlight plasma that is flowing towards the observer; red areas highlight plasma that is flowing away from the observer, (c) full-sun plasma composition map created from the ratio of the Si X $258.37 \AA$ and S X $264.22 \AA$ spectral lines; darker areas correspond to regions with photospheric abundances; lighter areas correspond to regions with enhanced (coronal) abundances. From Brooks et al (2015).

region ages. Baker et al (2015) found that the active region stayed coronal-like only in the core region. The surroundings were affected by continuous small-scale flux emergence, which changed the abundance in those regions to photospheric-like.

Quasi-periodic propagating disturbances (PDs) are seen in coronal polarized brightness intensity image sequences, and there is evidence that these may be slow-mode magnetoacoustic waves propagating into the corona (e.g., Ofman et al, 1997). The PDs were also seen in Hinode/EUV Imaging Spectrometer observations, and interpreted as slow magnetosonic waves (e.g., Wang et al, 2009a). The interpretation of the periodic disturbances in terms of slow magnetosonic waves was challanged by the detection of asymmetric Doppler shifts (blue) in the source regions (De Pontieu and McIntosh, 2010). Tian et al (2011) analyzed EIS and XRT data using EIS sit and stare data to maximize the time cadence. They found that repetitive high velocity upflows could be responsible for the oscillatory behavior observed. Further observational studies using Hinode/EIS data related between Alfvénic waves and flows (Tian et al, 2012). Evidently, the interpretation of the observed PDs is not straightforward, and recent 3D MHD modeling helped understand the relation between the chromospheric upflows and the slow magnetosonic waves that can be produced by these quasi-periodic upflows in the very low corona and propagate to higher altitudes in the corona (Ofman et al, 2012; Wang et al, 2013). The modelling suggests that the observed propagating disturbances and the associated upflows may be produced by nanoflares at the coronal loops footpoints in the corona/transition region interface and hence both waves and flows may contribute to the PDs at lower heights. This idea was supported by a recent analysis of SDO/AIA data, where PDs were shown to be closely related to chromospheric upflows (Jiao et al, 2015).

The origin of these upflows and their role in the mass supply to the corona is still being debated (e.g., Klimchuk, 2012). The possibilities that have been put forward are either from lower atmosphere in the chromosphere, or higher up in the corona. De Pontieu et al (2009) found a correlation between the emission line asymmetries 
in the corona and the dynamics in type II spicules in the chromosphere. He et al (2010) also found a relationship between chromospheric jets and coronal upflows. Other studies have indicated that the source is more likely to be in the corona. Harra et al (2008) found that there are large loops connecting from the active region to a nearby bipole through magnetic modeling indicating that the upflows are due to expansion of large loops. Boutry et al (2012) studied the same region and found the connection between the regions concluding that upflows originate from either large-scale loops or open magnetic field structures. Upflow regions were found to lie along regions where the magnetic field displays strong gradients of magnetic connectivity (quasi-separatrix layers) by Baker et al (2009). This suggests that magnetic reconnection between these open and closed magnetic fields drive the upflows. Comparisons have also been made between the upflows and radio noise storms by Del Zanna et al (2011).

Considering the small field of view of Hinode/EIS, it is challenging to make a direct link to the solar wind and therefore to determine whether these upflows actually become outflows leaving the Sun. Slemzin et al (2013) aimed to link the EIS data to large-scale structures that are observed at more than one solar radius. The location of the EIS upflows and the in-situ data of ACE were found to be consistent, by using a potential-field source-surface model, with the active region as a source of the solar wind. Modeling was carried out by van Driel-Gesztelyi et al (2012) both on a local and a global scale to determine which of the upflows could potentially form outflows seen in the solar wind. They found that it was the upflows of an active region that had a coronal hole as a neighbor. Edwards et al (2015) carried out a study of seven active regions along with global potential magnetic field extrapolations. In most cases the upflows do not correspond to a region of open field at all. The active region that did have open field related to the upflows was located alongside a coronal hole. The other regions of upflow were related to large-scale closed loops. All the upflows however were intersected by separatrix surfaces associated with null points located high in the corona, which could indicate important reconnection sites. Fazakerley et al (2015) studied a full Carrington rotation (CR) to determine the sources of slow and fast wind. There are three periods of enhanced velocity solar wind, which are related to active regions located beside coronal holes. Evidence is accumulating that a coronal hole located near an active region can affect whether the upflows from the edges of an active regions can become solar wind outflows. There have been detailed studies of an active region (Neugebauer et al, 2002) that also show how upflows can become outflows even with no associated coronal hole. This can occur through a more complex two-stage reconnection process (Culhane et al, 2014; Mandrini et al, 2014).

\subsubsection{Some results from $S O H O / U V C S$ instrument}

UVCS spectroscopic observations have significantly contributed to our understanding of streamers and slow wind since the beginning of the SOHO mission (see also Antonucci (2006) and Kohl et al (2006) for reviews of UVCS results). The hydrogen Ly- $\alpha$ and O VI emission lines were used to study streamers (see, e.g., Kohl et al, 1997; Noci et al, 1997b; Raymond et al, 1997; Stra- 
chan et al, 2002; Ko et al, 2008) and it was deduced that the properties of the $\mathrm{O}^{5+}$ ions, such as their relative abundances and temperatures, vary across the streamer structure in a qualitatively different way than the properties of protons and electrons. In particular, one of the first results was the discovery that quiescent streamers have a marked heavy ion (such as $\mathrm{O}^{5+}, \mathrm{Mg}^{9+}$ and $\mathrm{Si}^{11+}$ ) depletion in their cores with respect to their bright lateral branches (e.g., Noci et al, 1997b; Marocchi et al, 2001; Uzzo et al, 2003). The core dimming in the O VI $103.2 \mathrm{~nm}$ and $\mathrm{Mg}$ X $62.5 \mathrm{~nm}$ line has been recently analyzed by using a $2.5 \mathrm{D}$ multi-fluid model that demonstrated the effects of heavy ion gravitational settling, and cooling of the preferentially heated heavy ions through the interaction with electrons and protons producing core dimming (Ofman et al, 2011, 2013). Several different explanations for this feature have been proposed also related with the SSW origin (e.g., Noci et al, 1997a; Raymond et al, 1997; Ofman, 2000; Frazin et al, 2003; Akinari, 2007). The present review is not related directly to this point, but we would like to point out that this important observable is not due to a temperature effect (Noci et al, 1997a). In addition, the line width of the O VI emission is broader than the Ly- $\alpha$ line width, and this difference is larger outside the streamer than in the core (see Fig. 3). The outflow speed of the $\mathrm{O}^{5+}$ ions was studied and found to increase from being negligible in the core to significant values outside the streamer (see Fig. 4).

Important information on the SSW origin can derive from the microscopic velocity distributions of the heavy ions in three dimensions. The velocity distribution along the two directions perpendicular to the radial is assumed to be the same. The kinetic temperature, $\mathrm{T}_{k}$, inferred from the spectral line width observed by UVCS, is a measure of the velocity distribution width along the LOS, so this is named in the following as perpendicular (to the radial and also to the assumed magnetic field direction) kinetic temperature. UV spectroscopy of streamers shows that heavy ions such as $\mathrm{O}^{5+}$ have higher perpendicular kinetic temperatures than the protons particularly in coronal holes (see Kohl et al, 1997; Cranmer et al, 1999b). While the signatures of the protons cannot be measured directly, they are coupled to $\mathrm{H}$ atoms by charge exchange up to several solar radii (e.g., Withbroe et al, 1982). The kinetic temperatures of $\mathrm{O}^{5+}$ and $\mathrm{H}$ can be determined from the line widths of the coronal O VI $103.2 \mathrm{~nm}$ and H I Ly $\alpha$ emission. These temperatures include the line of sight thermal motions of the atoms/ions plus any unresolved non-thermal motions caused by waves or turbulence. Therefore, in order to determine the 'true' kinetic temperature, the effects of the unresolved wave or fluid motions must be subtracted (e.g., Ofman and Davila, 1997).

Figure 3 shows the results of SOHO/UVCS determinations of perpendicular kinetic temperatures, $\mathrm{T}_{\perp}$, for $\mathrm{H}$ and $\mathrm{O}^{5+}$ inside and in the external parts of coronal streamers. These measurements are from many observations mainly at the solar minimum period from both helmet streamer and pseudo-streamer (Kohl et al, 1997; Poletto et al, 2002; Strachan et al, 2002; Frazin et al, 2003; Antonucci et al, 2005; Susino et al, 2008; Abbo et al, 2010a, 2015). It is clear that for heights above $2 \mathrm{R} \odot, \mathrm{T}_{\perp}\left(\mathrm{O}^{5+}\right)$ is rapidly increasing while $\mathrm{T}_{\perp}(\mathrm{H})$ is nearly constant or slightly decreasing. Measurements along the streamer axis are color coded for each species (green for $\mathrm{O}^{5+}$ ions and red for $\mathrm{H}$ neutral atoms). The electron temperatures (blue symbols), $\mathrm{T}_{e}$, are calculated by Fineschi et al (1998) from the profile of the 
electron scattered H I Ly $\alpha$ component as derived from UVCS data, and by Li et al (1998) from an emission measure analysis. At low heights, within the closed field regions of the streamers, the plasma is isothermal with electrons, $\mathrm{H}$, and $\mathrm{O}^{5+}$ all having the same temperature. Along the streamer axis, the temperatures start to diverge at $2 \mathrm{R}_{\odot}$ and above. However, the temperatures of the different species are already different at $1.5 \mathrm{R} \odot$ for the measurements in black that were sampled near the streamer edges/coronal hole boundaries. The fact that the ion temperature profiles are rising rapidly and the electron and $\mathrm{H}$ (or proton) temperatures are not decreasing very fast suggests that continual, extended heating is taking place.

The main assumptions for the velocity distribution along the radial direction are: an isotropic Maxwellian velocity distribution for the electrons and the ions along the streamer axis with the width defined by the observed $\mathrm{T}_{k}$, and a bi-Maxwellian velocity distribution for the ions in the streamer edges. In this last case, the ion kinetic temperature corresponds to the observed line width in the plane perpendicular to the radial direction, and the ion kinetic temperature along the radial direction is equal to the electron temperature (maximum anisotropy). The ion temperature anisotropy assumption is dictated by the fact that the line broadening in the streamer edges is about a factor 2-10 larger than inside the streamer core, thus representing an intermediate state between closed field regions and the cores of coronal holes, where the ion velocity distributions are found to be highly anisotropic (e.g., Kohl et al, 1998; Cranmer et al, 1999b). This determines another relevant point which is the temperature anisotropy $\left(T_{\perp} / T_{\|}\right)$found not only in coronal holes (e.g., Cranmer, 2009), but also in the streamer edges and coronal hole boundaries with values in the range of 1.3-2 (Frazin et al, 2003; Susino et al, 2008). In Fig. 3, the fact that the $\mathrm{O}^{5+}$ perpendicular temperatures are rising rapidly and the electron and $\mathrm{H}$ (or proton) temperatures are not decreasing very fast suggests that continual, extended heating is taking place and that the process that leads to large perpendicular heating may be important for the acceleration of slow (as well as the fast) solar wind. Such preferential heating process, could be the result, for example, of energy dissipation through ion cyclotron resonance of high-frequency (left-hand polarized) Alfvén waves (e.g., see the review by Ofman, 2010).

Figure 4 shows empirical measurements for coronal outflow velocities as a function of heliocentric height obtained by applying the Doppler dimming technique based on the ratio of O VI line intensities (Beckers and Chipman, 1974; Noci et al, 1987). It is interesting to note that the outflow velocity measurements made along the streamer axis (green symbols) are greater than zero in the region where there are high ion kinetic temperatures. The same condition applies for locations that are external to the streamer edges (black symbols). Almost every place where there is a measurable outflow, the $\mathrm{T}_{\perp}\left(\mathrm{O}^{5+}\right)$ is larger than $\mathrm{T}_{\perp}(\mathrm{H})$. Antonucci et al (2005) found that the kinetic temperature of hydrogen remains two order of magnitude lower than that of oxygen in coronal holes and one order of magnitude lower in equatorial streamers. Antonucci et al (2005), Susino et al (2008), and Abbo et al (2010a) found that the main source for the SSW is the coronal hole/streamer boundary region (black symbols in Fig. 4), where the wind velocity depends on the magnetic field topology of flux tubes. Abbo et al (2010a) adopted an improved version of the Doppler dimming technique that is used to 
derive both electron density and outflow velocity of the expanding corona from the O VI 103.2-103.7 nm line intensities, given constraints on the geometry of flow tubes connecting corona and heliosphere.

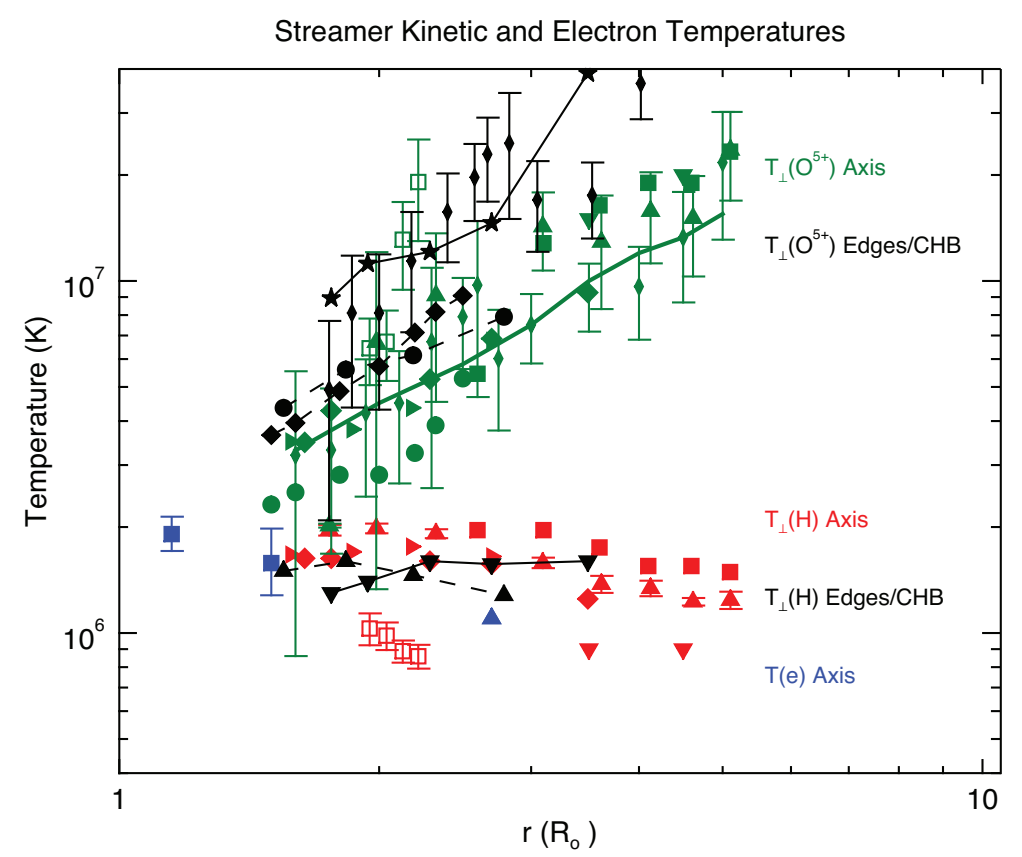

Fig. 3 A summary of UVCS measurements of coronal temperatures (as a function of projected height from Sun center) for $\mathrm{H}, \mathrm{O}^{5+}$ and electrons, for different streamer regions. In general $\mathrm{O}^{5+}$ kinetic temperatures are higher than those for $\mathrm{H}$ and electrons. Along the streamer axis, the temperatures are color-coded: red for $\mathrm{H}$, green for $\mathrm{O}^{5+}$, and blue for electrons. Temperatures near the streamer edge/coronal hole boundary are shown in black. Solid symbols are for helmet streamers and open symbols are for pseudo-streamers. Note that ion kinetic temperatures include both thermal and non-thermal components. These results are for a collection of streamers analyzed by different authors and observed from 1996 to 2008 close to solar minimum periods. See the text for more details. Sources for data in this figure are given below. Except where noted, all symbols are solid (filled). Abbo et al (2010a): green right triangles, red right triangles, black circles, black up-triangles; Abbo et al (2015): green squares (open), red squares (open); Antonucci et al (2005): green large diamonds, red large diamonds, black stars, black down-triangles; Fineschi et al (1998): blue up-triangle; Frazin et al (2003): green squares, red squares; Kohl et al (1997): green circles, black diamonds; Li et al (1998): blue squares; Poletto et al (2002): green down-triangles, red down-triangles; Spadaro et al (2007): dot-dash line; Strachan et al (2002): green up-triangles, red up-triangles; Susino et al (2008): green narrow diamonds, black narrow diamonds.

Strachan et al (2012) used synoptic maps of electron density and coronal outflow velocity at $\sim 2.5 R \odot$ to estimate flux tube expansion factors, $f$, as a function of latitude at solar minimum. The novelty of this work shows that flux tube expansion factors can be determined from the physical conditions in the corona, independent of magnetic field measurements. Preliminary results show that large $f$ is 


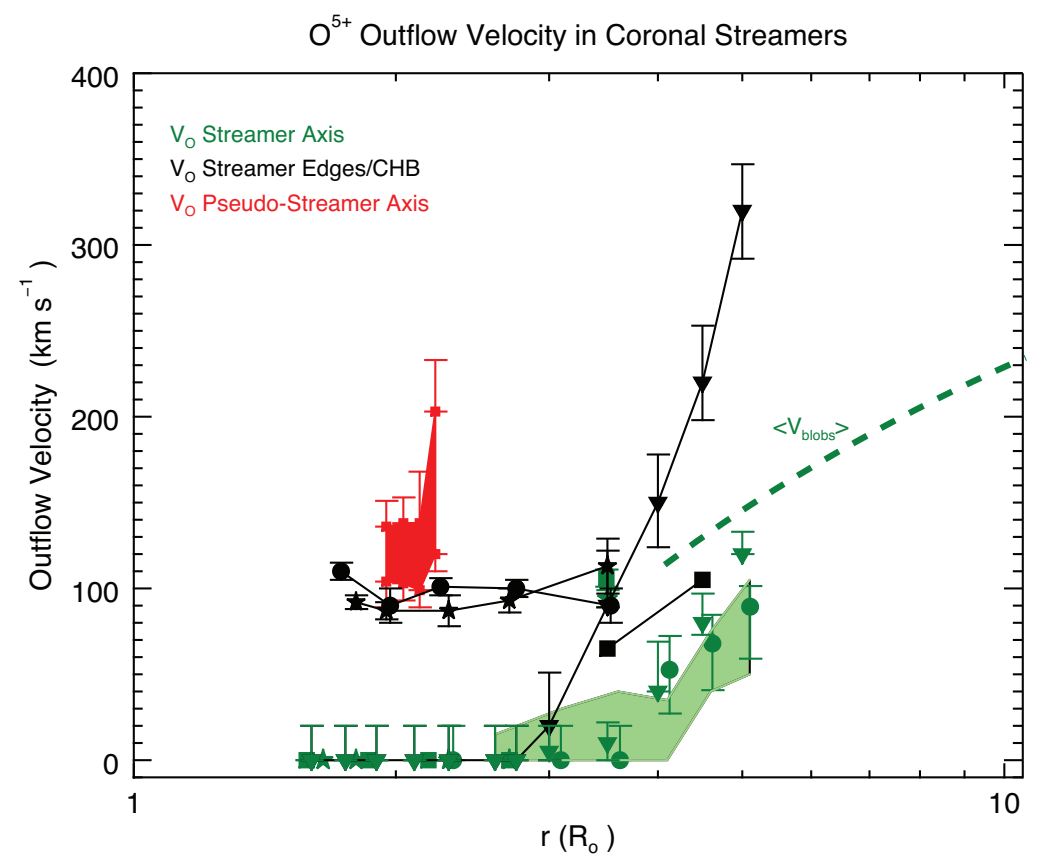

Fig. 4 A summary of UVCS $\mathrm{O}^{5+}$ outflow velocity measurements (as a function of projected height from Sun center) for different regions: (1) along the streamer axis (green symbols), (2) at the streamer edge/coronal hole boundary (CHB; black symbols),(3) along the pseudo-streamer axis (red area). Mean blob speeds from $\mathrm{SOHO} / \mathrm{LASCO}$ observations are added as a reference for outflow velocities determined by other techniques. See the text for more details. Sources for data in this figure are shown below. Abbo et al (2010a): green squares, black circles; Abbo et al (2015): red squares (solid red region); Antonucci et al (2005): green stars, black stars; Frazin et al (2003): solid green region; Poletto et al (2002): black squares; Strachan et al (2002): green circles; Susino et al (2008): green down-triangles, black down-triangles.

found at the edges of streamers where the SSW is thought to originate. Antonucci et al (2012) also found that, during solar minimum, when the coronal magnetic configuration is rather simple, the open magnetic fields emerging from the wide polar coronal holes channel both the fast and the slow wind: the fast wind flows along flux tubes with lower areal divergence than in the slow wind which is guided by flux tubes characterized by non-monotonic areal expansion functions (first a divergence and then a convergence).

Some authors showed that equatorial streamers at solar minimum exhibit plasma outflows along the streamer axis (green symbols in Fig. 4). Indeed, by determining a lower limit of the outflow speed derived from the O VI line ratios in the streamer region Noci and Gavryuseva (2007) found that SSW directly emerging from the streamers is likely confined to a thin layer near the current sheet. Nevertheless, the detection of multiple current sheets is not evident from present in-situ data that would have been difficult with Ulysses measurements due to limited time resolution. Moreover, Abbo et al (2015) showed with an analysis based on UV ob- 
servations of pseudo- or unipolar streamers that these structures can contribute to the SSW in non-bipolar magnetic field configuration (red area in Fig. 4). There are some clues that protons (which behave as neutral atoms due to charge exchange at distances of up to several solar radii) flow with lower velocities in coronal holes (e.g., Kohl et al, 2006) and in the external parts of streamers (Susino et al, 2008). Another possibility is that these outflows are strictly connected with the 'blobs' seen in white light observations (see Section 2.2) but these events describe a sporadic and intermittent emission of plasma, whereas from the UVCS observations the SSW is formed by a continuous outflow (although, the exposure time of UVCS images could reach many hours, that average out variability on shorter time scales).

In addition to providing plasma parameters (temperature, velocity, and density) in the solar wind source regions, UVCS spectroscopy was able to determine elemental abundances. It is known that elemental abundances vary among the different coronal structures with respect to photospheric values (e.g., see the review by Raymond et al, 2001, and references within). The determining parameter appears to be the FIP effect. This effect is much more pronounced in the SSW than in the fast solar wind (von Steiger et al, 2000) and therefore it can be very useful as a tracer of their sources. Measurements of enhanced abundances in streamers for low-FIP elements provide a direct connection between streamers and the SSW (e.g., Raymond et al, 1997). The enhancements of low-FIP elements in the edges of coronal streamers is similar to those found in the SSW (FIP-bias of $\sim 3$ ), which is a strong indication that at least some of the solar wind originates from these regions (Uzzo et al, 2003). Other authors found that open magnetic field regions outside streamers are characterized by oxygen ion abundance typical of the heliospheric SSW (Abbo et al, 2006; Antonucci et al, 2006), confirming that these regions are the dominant sources of the SSW. Ko et al (2008) provided 2D maps of coronal elemental abundances of streamers derived from observations of an east limb corona at $1.63 R_{\odot}$ from 2000 September 20 to October 1 , that were compared with large-scale coronal properties of a 3D MHD model in order to investigate their possible association with open and closed field structures. They found a good indication that the open field regions have lower density and higher abundance values than the closed field regions (see also Section 2.3 for further discussion on the FIP effect).

2.2 Imaging Data: some results from SOHO/LASCO and STEREO/COR Coronagraphs

White-light observations from the LASCO C2 coronagraph on SOHO have shown that helmet streamers are dynamical, non-steady structures with a strong tendency to expand outward, giving rise not only to large-scale "streamer blowout" CMEs, but also to small-scale plasma inhomogeneities ("blobs") that are continually emitted from their cusps (see Sheeley et al, 1997; Wang et al, 1998; Song et al, 2009). The streamer blobs are confined to the immediate vicinity of the heliospheric plasma/current sheet, which extends outward from the cusp and appears to consist of fine ray-like structures. As shown in Fig. 5, their speeds increase 
gradually from $\sim 0-100 \mathrm{~km} \mathrm{~s}^{-1}$ near the streamer cusp (located at a heliocentric distance of $r \sim 3 R_{\odot}$ ) to $\sim 300-400 \mathrm{~km} \mathrm{~s}^{-1}$ at $r \sim 30 R_{\odot}$ (the outer edge of the LASCO C3 field of view). In the interpretation of Sheeley et al (1997), the velocity profile of the blobs (which resembles that of slow streamer blowouts) reflects that of the surrounding SSW. Using the STEREO/COR1 coronagraph, Jones and Davila (2009) have also detected and measured the speeds of small density enhancements inside $r \sim 2 R_{\odot}$; however, many of the features that they tracked are likely to have been associated with CME eruptions rather than streamer blobs.

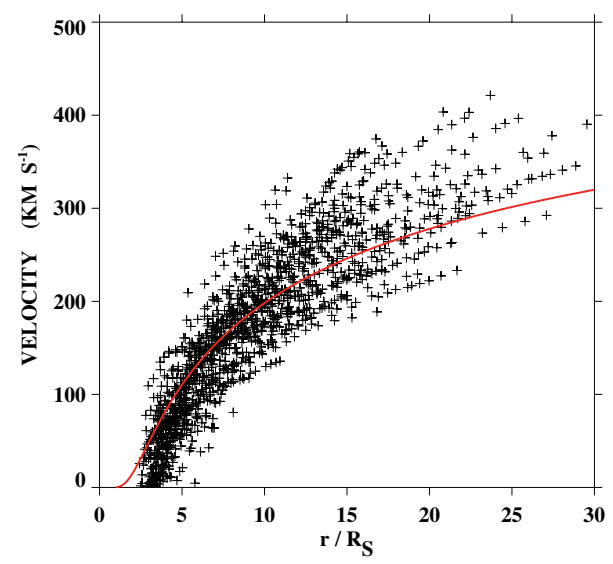

Fig. 5 Scatter plot of speed vs. heliocentric distance for 80 streamer blobs, whose height-time tracks were each fitted with a quadratic function. The sky-plane projected velocities increase monotonically from $\sim 0-100 \mathrm{~km} \mathrm{~s}^{-1}$ at $r \sim 3-4 R_{\odot}$ to $\sim 200-400 \mathrm{~km} \mathrm{~s}^{-1}$ at $r \sim 20 R_{\odot}$. Also plotted (red) for comparison is the Parker solution for a radially expanding, isothermal corona at a temperature of $1 \times 10^{6} \mathrm{~K}$.

More recent observations that exploit the different viewing angles afforded by STEREO have revealed that many, if not all streamer blobs are small flux ropes (Sheeley et al, 2009). Illustrative examples may be seen in Fig. 6. Here, COR2A looks down on the plasma sheet and sees arch-like structures, representing the case where the line of sight is perpendicular to the plane of the helical flux rope; COR2B looks edge-on at the plasma sheet and sees narrow, concave-outward structures, representing the case where the line of sight is along the axis of the flux rope. These observations suggest that the blobs are created not by interchange reconnection between streamer loops and open field lines, but rather by reconnection among the streamer loops themselves. In contrast, interchange reconnection provides a possible mechanism for maintaining the heliospheric plasma sheet (along which the blobs propagate), as well as accounting for its ray-like structure. STEREO/SECCHI observations show that the blobs may have quasi-periodic nature and their acceleration and expansion is self-similar (Viall and Vourlidas, 2015).

The helmet streamers that are the source of plasma blobs separate coronal holes of opposite polarity and extend outward to form the heliospheric current sheet. However, a significant fraction of the white-light structure in the outer corona is contributed by the plasma sheet extensions of "pseudo-streamers" which separate coronal holes of like polarity and do not have current sheets (see Fig. 13 for the 
sketch of the magnetic field configuration). The cusps of pseudo-streamers are usually located well below $r \sim 2.5 R_{\odot}$, unlike those of helmet streamers. Pseudo-streamers are generally far more quiescent than helmet streamers, and show fewer tendencies to expand outward unless an underlying filament erupts or an active region emerges beneath it. As illustrated in Fig. 2 by Wang et al (2012), pseudo-streamers do not normally emit blob-like ejecta.

The differing dynamical behaviors of helmet streamers and pseudo-streamers can probably be attributed to the fact that, whereas the magnetic field goes to zero above the cusp of a helmet streamer, it remains strong above the cusp of a pseudostreamer, where like-polarity open flux converges from both sides. As a result, the underlying loops have much less tendency to expand outward and pinch off in pseudo-streamers than in helmet streamers. This also makes it difficult to see how pseudo-streamers themselves can be a major source of SSW, other than that associated with their plasma sheets.

In summary, from recent papers on white light observations concerned with the SSW, we can deduce that:

- Heliospheric plasma sheet (along which the blobs propagate) consists of narrow rays formed by interchange reconnection at streamer cusps (Wang et al, 2012).

- Streamer blobs are mini flux ropes formed by pinching off closed loops in the intrinsically unstable cusp region (Sheeley et al, 2009) with evidence of quasiperiodicity (Viall and Vourlidas, 2015).

- Pseudo-streamers have plasma sheets (no current sheets) but do not emit blobs (Wang et al, 2007a, 2012).

2.3 In-situ data: some results from Ulysses/SWICS and ACE/SWICS instruments

More than four decades of space missions, e.g., IMP 8, ISEE 3, Helios, Wind, Ulysses, SOHO, ACE and STEREO, have produced a wealth of solar wind particle and magnetic field data measured in-situ. Planetary missions such as Voyager and MESSENGER have also provided solar wind measurements in-situ, covering a large range of heliospheric distance. These data have cumulatively contributed to our understanding of the solar wind in many aspects. In this section, we focus on the solar wind properties inferred from the Solar Wind Ion Composition Spectrometer (SWICS) instruments on Ulysses and ACE that measure the heavy ion composition. As mentioned in Section 1, the solar wind heavy ion charge states and elemental compositions are closely related to the properties of the solar wind source region, thus providing the diagnostics of SSW sources. The following two paragraphs further explain why this is so.

The solar wind ions gyrate, and flow out of the Sun along open field lines through the coronal environment where the density decreases with height. The evolution of the ionic charge states with the flow 

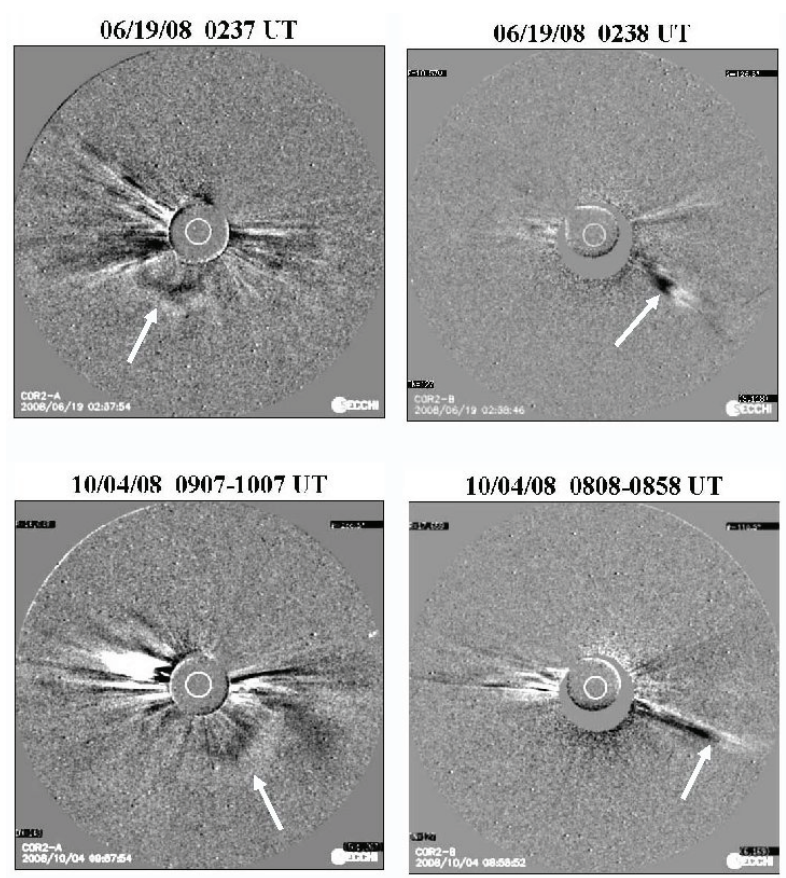

Fig. 6 Streamer blobs observed simultaneously from COR2A (left panels) and COR2B (right panels). In both the June 19 (top) and October 4 (bottom) 2008 events, COR2A looks down at the heliospheric plasma sheet, while COR2B views it edge-on. Field of view is from 2 to 15 $R_{\odot}$.

then undergoes a non-equilibrium ionization process that results in the freezing-in of the ions, i.e., the ionic charge states stop changing beyond some distance. This freezing-in process depends on the electron density, ion outflow speed and the ionization and recombination rates (thus the electron temperature) of the ions, and was found to complete within a few solar radii (e.g., Owocki et al, 1983; Buergi and Geiss, 1986). There are processes that can affect the ionic charge states in the solar wind flow, thus the inference from the data, such as non-thermal electron velocity distribution (Owocki and Scudder, 1983; Ko et al, 1996; Laming and Lepri, 2007) and differential ion flows (e.g., Ko et al, 1998; Esser and Edgar, 2001; Chen et al, 2003). Recently, there were series of efforts further refining the diagnostics using the ionic charge states (Landi et al, 2012a,d). It is worth noting that the coronal profiles (i.e., electron temperature, density and ion outflow) usually cannot be derived from the solar wind ionic charge states in a straightforward manner and need to be calculated empirically (e.g., Ko et al, 1997), preferably with as many constraints as possible provided by other data sources, such as spectroscopic observations (Landi et al, 2012b,c). On the other hand, solar wind ionic charge states provide stringent tests of solar wind models since the predicted frozen-in ionic charge states 
forward-modeled with the modeled coronal profiles must agree with the data (Zurbuchen et al, 2012; Landi and Testa, 2014).

The fact that the slow and fast solar wind exhibits different FIP biases implies that this difference is set at the chromospheric level where the elements with FIP of less than about $10 \mathrm{eV}$ are photoionized by the solar Ly $\alpha$ radiation followed by certain ion-neutral fractionation process such as neutral atom diffusion (von Steiger and Geiss, 1989; Peter, 1998), wave heating of ions (Schwadron et al, 1999), or waveinduced pondermotive force (Laming, 2004, 2009, 2015). Therefore the solar wind elemental abundances, when combined with abundance observations at the Sun can provide information on where and how the solar wind is formed. For example, the similar FIP bias between the slow wind and coronal loops lends support for the scenario that the slow wind is produced via interchange reconnection that releases plasma in coronal loops into the solar wind/open field (Fisk and Schwadron, 2001) (also see Section 2). This scenario would also introduce variations that should relate to variations in coronal loops. For example, the location of the interchange reconnection could affect the slow wind abundance, since the abundance can be modified in coronal loops such as by gravitational settling (Raymond et al, 1997; Ko et al, 2002; Weberg et al, 2015).

\subsubsection{Ulysses}

Ulysses was the first space mission to orbit the Sun on a high-inclination orbit (Wenzel et al, 1992). Launched in 1990 and injected into its unique orbit by a Jupiter fly-by in 1992, it operated for nearly three orbital periods until it was switched off in mid-2009. Thanks to a fortuitous timing its first orbit coincided with the solar activity minimum between sunspot cycles 22 and 23 , the second one with the solar maximum 23, and the third with the peculiar solar minimum 2324. It was no small surprise when it was found that the high-latitude heliosphere at solar minimum is completely dominated by a remarkably uniform high-speed stream emanating and superradially expanding from the polar coronal holes (McComas et al, 1998). Results from the SWICS sensor (Gloeckler et al, 1992) firmly confirmed the fact that the solar wind is a two-state phenomenon with a remarkably sharp boundary between them (Geiss et al, 1995b). In the meantime it was demonstrated that it would be better, or more physical, to differentiate the two states based on the temperature of their source regions as it is imprinted in the charge states of heavy ion species such as C, O, or Fe (von Steiger et al, 2000). It is important to recognize this aspect and the value of composition instrumentation in future space missions.

One of the principal charge state ratios to separate "fast" and "slow" wind is $\mathrm{O} 76=\mathrm{O}^{7+} / \mathrm{O}^{6+}$, simply because oxygen is the most abundant heavy ion ( $\mathrm{A}$, the mass number, $>4)$ and $6+$ is its dominant charge state under most solar wind conditions. This parameter has the (small) disadvantage that $\mathrm{O}^{7+}$ is very scarce in "fast" wind from the cool coronal holes so it gets count limited. Therefore it is a good practice to complement $\mathrm{O} 76$ with $\mathrm{C} 65=\mathrm{C}^{6+} / \mathrm{C}^{5+}$. The two parameters track each other very nicely as is readily evident from Fig. 7 , which is a contour plot of all daily averages during the entire mission. Obviously the data fall into two very clearly separated categories, one at low C65 and low O76 that mark "fast" 
wind from cool coronal holes and the other at high values for the "slow" wind from the streamer belt. The red separatrix between these categories is given by the equation $O 76 \times C 65=0.01$ and we propose to use this as a robust measure for solar wind category separation (von Steiger et al, 2010). However we must qualify this statement to apply primarily to the Ulysses mission, which was dominated by fast streams from polar coronal holes during two of its three orbits. A more elaborate method may be needed for observations with dominance of equatorial coronal holes such as the ones from ACE (see next subsection).

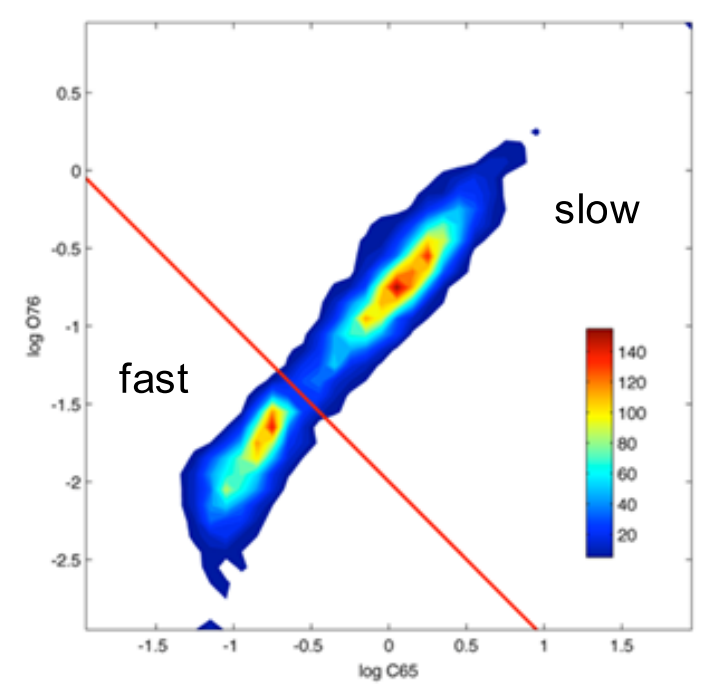

Fig. 7 Contour plot of all daily averages of the $\mathrm{C}^{6+} / \mathrm{C}^{5+}(\mathrm{C} 65)$ ratio vs. the $\mathrm{O}^{7+} / \mathrm{O}^{6+}(\mathrm{O} 76)$ ratio obtained with Ulysses-SWICS. The two ratios are very strongly correlated and together serve as ideal, physical parameters to separate the "slow" from the "fast" solar wind. The dividing line (red) is given by $O 76 \times C 65=0.01$ (figure adapted from von Steiger (2008).

Closer inspection of Fig. 7 reveals that the two solar wind types are not equally distributed: the "fast" wind seems to be more concentrated than its "slow" counterpart. This was investigated in more depth by von Steiger et al (2010), who analyzed the oxygen flux from the SWICS sensor in comparison to the proton flux from the SWOOPS sensor (Bame et al, 1992). The comparison only became sufficiently reliable once all systematic uncertainties of the heavy ion identification in SWICS were understood and quantified, as documented in von Steiger and Zurbuchen (2011). The daily values of the $\mathrm{O}$ and $\mathrm{H}$ fluxes were scaled to $1 \mathrm{AU}$ and separated in "fast" and "slow" as described in the previous paragraph. Their distributions are given in Fig. 8 as blue and red histograms for the "fast" and "slow" wind, respectively. In particular, the top panel gives the velocity distributions of the daily averages, indicating that for Ulysses data fast-slow and the charge state classification of the two solar wind types are not all that different (this is much less the case for ACE data, though); the middle and bottom panels show the $\mathrm{O}$ and $\mathrm{H}$ flux observed with Ulysses-SWICS and SWOOPS, respectively. The Ulysses flux 

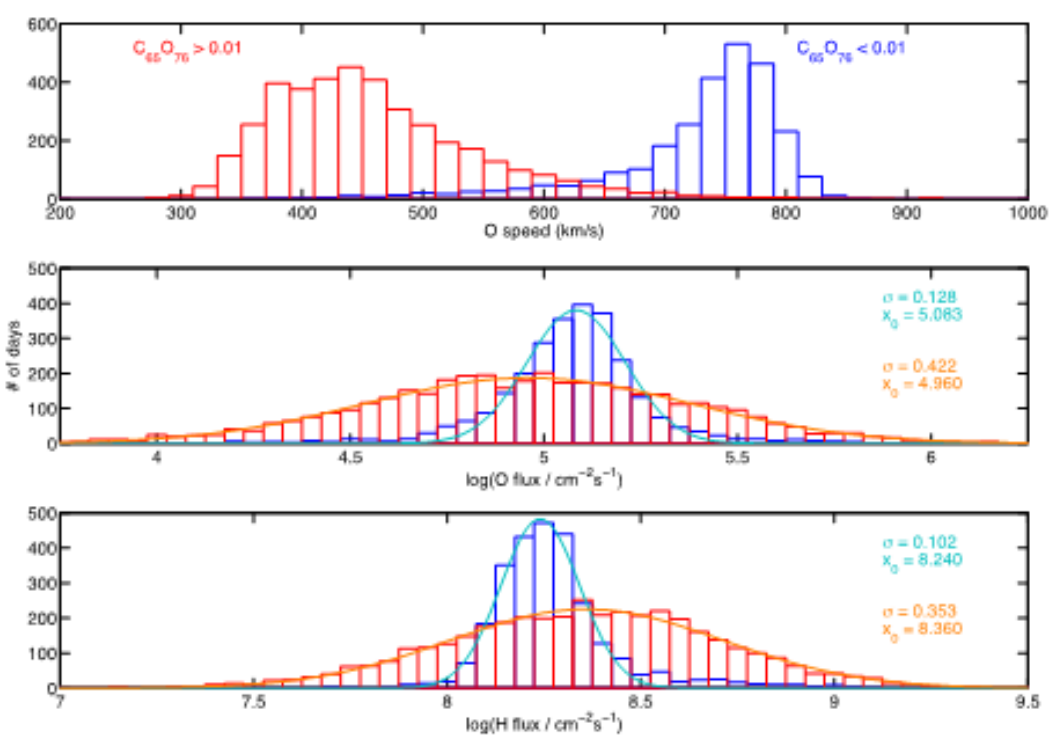

Fig. 8 Distribution of the daily averages of the solar wind speed (top), the flux of oxygen ions observed with Ulysses-SWICS (middle), and the proton flux observed with Ulysses- SWOOPS (bottom). The "fast" and the "slow" wind (blue and red, respectively) are both characterized by smooth lognormals, but the "slow" wind is 3 times as variable as the "fast" one (figures adapted from von Steiger et al (2010).

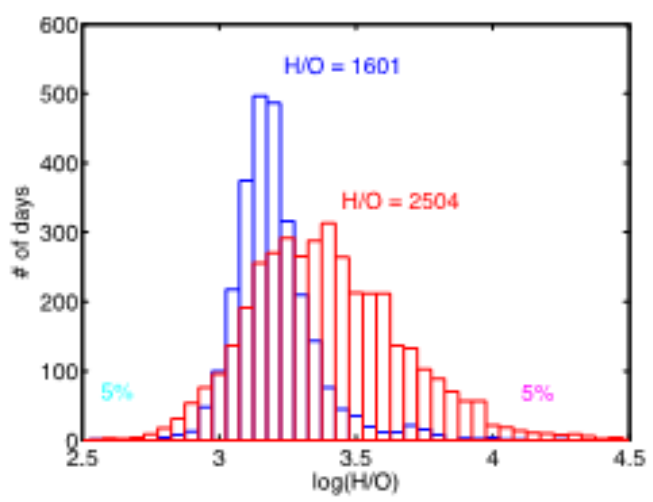

Fig. 9 Distributions of daily values of the H/O flux ratios in the "fast" (blue) and the "slow" (red) solar wind. The latter is found to be about twice as variable than the former (figures adapted from von Steiger et al (2010).

distributions are remarkably smooth log-normals (Burlaga and Lazarus, 2000) in all cases with a striking difference in the widths between the "fast" and the "slow" variant. Thus we may conclude that the "slow" solar wind is characterized not so much by its low speed, but by its variability. Both for $\mathrm{H}$ and for $\mathrm{O}$ fluxes, the "slow" wind distribution of daily averages is about three times wider than its 
"fast" counterpart. While the "fast" wind flux varies by $26 \%(\mathrm{H})$ or $33 \%(\mathrm{O})$, the slow wind does so by a factor of $2.3(\mathrm{H})$ or $2.6(\mathrm{O})$, respectively.

Of course flux variability does not necessarily imply composition variability. We have therefore calculated the daily averages of the oxygen abundance, or rather the one of its inverse, $\mathrm{H} / \mathrm{O}$, for convenience, and we have plotted its distribution in Fig. 9. Note that the width difference is a bit less than in the case of the fluxes, but we still find that the $\mathrm{O}$ abundance is twice as variable in the "slow" than in the "fast" wind. Likewise we find that Ne (Shearer et al, 2014) and all other heavy element abundances that can be determined with Ulysses-SWICS (von Steiger and Zurbuchen, 2016) are significantly more variable in the "slow" wind.

\subsubsection{ACE/SWICS}

Beyond the general properties of the slow wind that separate it from the fast wind, it is known that there are significant variations among individual solar wind streams. Figure 8 shows that ion fluxes and the $\mathrm{O} / \mathrm{H}$ abundance all exhibit a distribution of values, much more so in the slow wind than in the fast wind. As mentioned in the previous subsection, the clear separation between the slow and fast wind measured from Ulysses based on the criterion of $O 76 \times C 65=0.01$ might be partly due to large samplings of very high speed wind from the two large polar coronal holes during the two polar passes in 1995 and 2007. Indeed, the distribution from ACE data based on the same criterion yields a wider distribution of the solar wind speed in the "fast" category with occurrences peaking at much slower speed of $600 \mathrm{~km} \mathrm{~s}^{-1}$, and the overlap with the "slow" category is larger (Zhao and Landi, 2014). As clearly shown in Fig. 10, there are substantial variations in the solar wind charge states and abundances even at any narrow speed range. Such variations must be largely caused by variations in the solar source region properties and the processes that produce them, either from intrinsic differences among coronal holes at one time or relating to long-term temporal trend in a solar cycle.

It is evident that: 1) there are substantial range of variation in the heavy ion composition among the solar wind streams at any time in a solar cycle, more so in the SSW (e.g., Lepri et al (2013), cf. Figs. 8 and 9). The 076 ratio (also the charge states of $\mathbf{C}$, not shown here) has a clear correlation with the solar wind speed, but not for the case of the average charge of $\mathrm{Fe}$ and the $\mathrm{Fe} / \mathrm{O}$ abundance ratio (the middle and right panels). 3) There are long-term trend in a solar cycle (cycle 23 in this case) that, from solar maximum to minimum, the mean ionization states decrease across all solar wind peed. However, for the slowest speed of $300-400 \mathrm{~km} \mathrm{~s}^{-1}$, the $\mathrm{Fe} / \mathrm{O}$ ratios in solar minimum still retain similar occurrence in high values as in the solar maximum. It is worth noting that the slow wind $\mathrm{He} / \mathrm{H}$ abundance ratios analyzed in Kasper et al (2012) exhibit a different temporal behavior between these sub-groups of the slow wind. In contrast to the FIP bias, the abundance ratio of $\mathrm{Ne} / \mathrm{O}$ (both are highFIP elements) in the slow wind exhibits an opposite temporal trend in the solar cycle that Ne/O increases from solar maximum to minimum (Shearer et al, 2014). We also want to point out that the general notion of higher $\mathrm{Fe} / \mathrm{O}$ (thus larger 

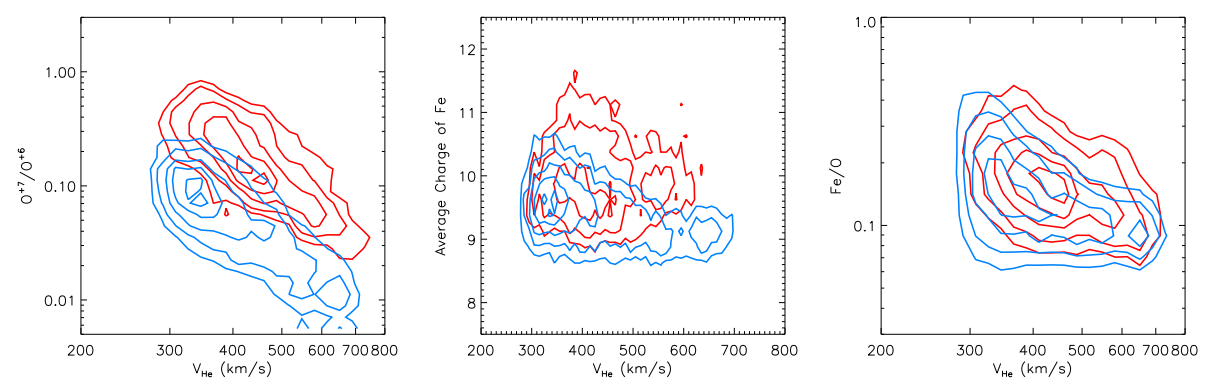

Fig. 10 Contours of 'fraction of occurrence' for the $\mathrm{O}^{7+} / \mathrm{O}^{6+}$ density ratios (left panel), average charge of $\mathrm{Fe}$ (center panel) and $\mathrm{Fe} / \mathrm{O}$ elemental abundance ratios (FIP bias) versus the solar wind helium speed for years 1999-2000 (in solar maximum, red contours) and years 2008-2009 (in solar minimum, blue contours). In order to compare between the two time periods, the contour levels represent the same fractions for both. The plots are constructed from ACE/SWICS 1.1 level-2 1-hour data excluding ICME-type periods as identified in the released data. The linear Pearson correlation coefficients (calculated from the 1-hr data for each periods) are noted on the top of the plots.

FIP bias) in the slow wind than in the fast wind is only valid when averaging over a large ensemble of solar wind streams. There are many slow speed winds that have low FIP bias (i.e., Fe/O close to its photospheric ratio of $\sim 0.06$ ). The distribution of the Fe charge states also has this similar behavior. This is in contrast to the $\mathrm{O}^{7+} / \mathrm{O}^{6+}$ ratio which exhibits a clear anti-correlation with the solar wind speed. Such variation is a reflection of the coronal condition where the solar wind is formed. To understand the SSW sources, the next sensible step would be to investigate how these ion composition properties in individual streams relate to their respective source regions. One obstacle in achieving this goal that still remains is the uncertainty of present coronal field models, that can not accurately pinpoint the footpoint locations of particular solar wind streams.

Figure 11 shows a specific example for how different individual SSW streams can be. The two consecutive solar wind streams originated from two coronal holes of opposite polarity about 60 degrees apart in longitude, and were separated by a heliospheric current sheet. It is apparent that the slow solar wind properties from these two coronal hole sources are very different even though they are at a similar speed. The first slow wind stream (DOY 313-315, blue-shaded), as compared to the second one (DOY 315-320, yellow-shaded), has similar proton density, lower proton temperature, smaller interplanetary magnetic field (IMF) and photospheric field strengths, higher $\mathrm{O}^{7+} / \mathrm{O}^{6+}$ ratios and Fe charge states, smaller $\mathrm{Fe} / \mathrm{O}$ abundance ratios but larger helium-to-proton ratios $(\mathrm{He} / \mathrm{H})$. Variations seen in these properties, whether from individual source regions or from a longterm trend in a solar cycle, provide a wealth of clues and should all be taken into account in understanding how the SSW is formed. See, Neugebauer et al (2002); Liewer et al (2004) for additional examples.

Zhao et al (2009) categorized the slow wind by the $\mathrm{O}^{7+} / \mathrm{O}^{6+}$ ion density ratios of 0.145 and above, excluding ICME periods when very high ionic charge states are common. This work was based on the ACE/SWICS measurements during solar 

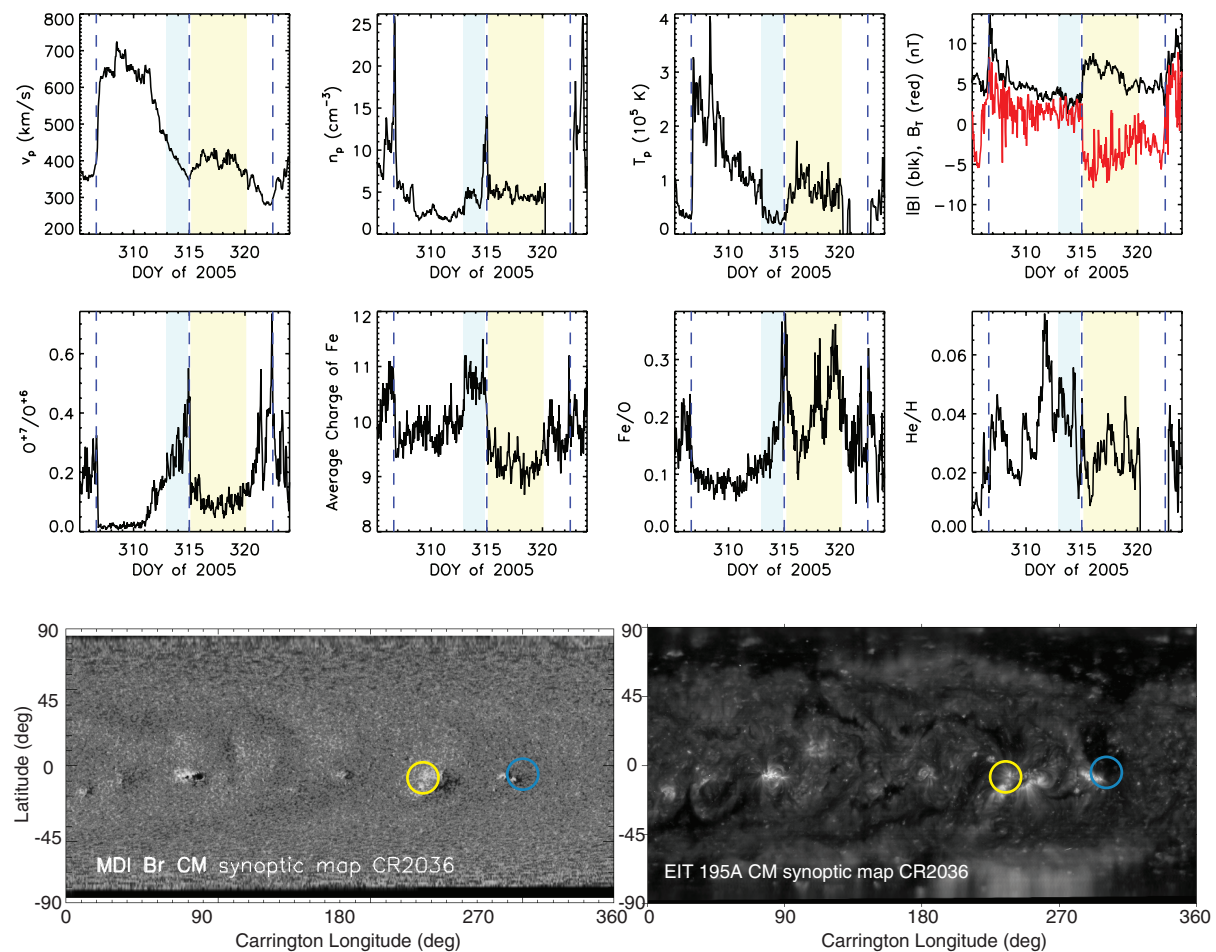

Fig. 11 Top two rows: solar wind data during November 2005 (DOY305-324) showing two consecutive solar wind streams (between the dashed lines) from two coronal holes separated by $\sim 60$ degrees in longitude. Panels in the top row from left to right are: proton speed $\left(n_{p}\right)$, proton density $\left(n_{p}\right)$, proton temperature $\left(T_{p}\right)$, and the IMF strength $(|B|)$ (black) and its component $B_{T}$ (red) in the RTN coordinate. The sign change in $B_{T}$ on DOY 315.0 and 322.5 indicates a crossing of the heliospheric current sheet, thus the likely change from one solar source $\mathrm{CH}$ to the other $\mathrm{CH}$ with opposite polarity. There is a data gap in $n_{p}$ and $T_{p}$ between DOY 320 and 323. Panels in the middle row from left to right are: the $\mathrm{O}^{7+} / \mathrm{O}^{6+}$ ratio, average charge of $\mathrm{Fe}$, $\mathrm{Fe} / \mathrm{O}$ elemental abundance ratio, and the helium-to-proton density ratio $(\mathrm{He} / \mathrm{H})$. Intervals in blue and yellow shades are the two slow wind intervals (DOY313-315 and DOY315320) for comparison. Bottom panels: SOHO MDI (left) and EIT $\lambda 195$ (right) synoptic maps for the corresponding CR \#2036. The blue and yellow circles mark the approximate footpoint locations for the first and second slow wind streams, respectively, based on the PFSS model. The footpoint magnetic field strength (also based on the PFSS model) for the second slow wind stream is about 5 times stronger than that for the first stream. Data are from the ACE/MAG, ACE/SWEPAM, and ACE/SWICS level-2 1-hour data. The MDI synoptic map is obtained from http://soi.stanford.edu/magnetic/index6.html.

cycle 23 (1998-2008). It is then realized that during the deep minimum of cycle 23 (2009-2010), many of the slow wind streams have $\mathrm{O}^{7+} / \mathrm{O}^{6+}$ ratios lower than this 0.145 threshold. Schwadron et al (2011); von Steiger and Zurbuchen (2011); Lepri et al (2013); Ko et al (2014) all reported that the solar wind ionization states in both the fast and slow wind were decreasing during the declining phase of cycle 23, which should be in some way related to the decreasing solar magnetic field (as well as the IMF) since less magnetic energy would be available for coronal heating (Schwadron et al, 2011). Ko et al (2014) further showed that 
this trend of decrease in the solar wind ionization states is different between polar coronal hole sources and equatorial coronal hole sources. They selected 27 solar wind intervals that originate from polar coronal hole sources (including the low-latitude extension) during 2004 -2008 and investigated the correlation among the solar wind ion charge states and the coronal hole properties such as the photospheric magnetic field and coronal electron temperature. They found that $\mathrm{C}$ and $\mathrm{O}$ ions that freeze-in low in the corona have better correlation with the photospheric magnetic field strength (both signed and unsigned) and the coronal electron temperature in the slow wind. On the other hand, Fe ions that freeze-in higher in the corona have better correlation with the two parameters in the fast wind instead. Such correlations with the photospheric magnetic field exist whether it is inside the coronal hole or at its outer boundary. However, good correlation with the coronal electron temperature only exists at the outer boundary of the coronal hole (Ko et al, 2014).

The above results indicate that the heat deposition would be at lower (higher) height for the slow (fast) wind, assuming that the amount of energy to heat the solar wind is positively correlated with the strength of the photospheric magnetic field. This agrees with Withbroe (1988) that the slow wind is associated with heating near the coronal base along rapidly diverging flux tubes, while the fast wind is associated with heating over an extended distance beyond the sonic point $\left(\gtrsim 2 R_{\odot}\right)$ in open field regions with small field expansion factor. However, the work of Ko et al (2014) could not judge whether the slow wind originates from the inside or outside of the coronal hole. Landi and Testa (2014) analyzed the electron density and temperature at the base of 60 quiescent streamers during 1996 to 2013 and found no temporal variation like that in the ion charge states. Regardless of the scenarios for the slow wind formation, this insensitivity of coronal properties in both coronal holes and streamers to the long-term trend of solar wind charge states in the solar cycle requires further work to reconcile. We caution that the charge state evolution with the solar wind flow is dictated by coronal properties in the open field lines, while the line emission from which the density and temperature were derived in the above-mentioned studies may be dominated by close loop sources which could mask the emission from the adjacent open field lines.

Recently, Fu et al (2015) identified the coronal sources of the solar winds sampled by the ACE spacecraft during 1999 - 2008 and examine the in situ solar wind properties as a function of wind sources on the basis of their speeds and $\mathrm{O}^{7+} / \mathrm{O}^{6+}$ ratios. This statistical study indicates that during the maximum phase, the majority of the solar wind as measured by ACE originates from active regions and that quiet Sun regions are the primary supplier during the minimum phase. This may imply that during the minimum phase, there are many small coronal holes (i.e., open fields) embedded within the quiet Sun regions. Such small coronal holes would only produce SSW and are too small to be seen by the EUV imaging instruments as "traditional" coronal holes with a clear boundary with the quiet Sun region. Ko et al (2014) showed that the coronal holes in the declining phase tend to be more bi-polar (i.e., larger fraction of small coronal loops) toward the solar minimum (see Fig. 9 of that paper). They speculate that these small slow wind source coronal holes may, alternatively, have brightness close to that of the small closed loops carpeting the quiet Sun, thus they are not easily distinguishable. 
D'Amicis and Bruno (2015) have recently studied the Alfvénic fluctuations of the SSW, common feature in the solar wind but found especially in the trailing edges of fast wind streams. The slow wind usually has a lower degree of Alfvénicity, being more strongly intermixed with structures of non-Alfvénic nature. They showed the first evidence in the interplanetary space of two different kinds of SSW: one coming from coronal streamers or active regions and characterized by non- Alfvénic structures and the other one being highly Alfvénic and originating from the boundary of coronal holes. Similar conclusions have been obtained by Wang et al (2009b) by comparing ACE solar wind data during 1998-2007 with extrapolations of the observed photospheric magnetic field. They identify two main components of the slow wind. One emanating from small coronal holes near active regions (at solar maximum) characterized by particularly strong low-coronal heating, high $\mathrm{O}^{7+} / \mathrm{O}^{6+}$ and $\mathrm{Fe} / \mathrm{O}$ ratios, large expansion factors, strong footpoint fields, and high mass and energy flux densities at the coronal base. The other (at solar minimum) coming from just inside the polar-hole boundaries and characterized by weaker low-coronal heating and intermediate $\mathrm{O}^{7+} / \mathrm{O}^{6+}$ and $\mathrm{Fe} / \mathrm{O}$ ratios.

\section{Numerical modeling}

How can the solar wind numerical models help us understand the sources and the acceleration mechanisms of the SSW, and how can the interaction between models and observations help to achieve these goals? This complex question is the topic of this section and we provide a brief overview of some present-day numerical modeling of the SSW from the transition region to the corona up to $10-20 R_{\odot}$, discussed during our ISSI Team meetings.

\subsection{Connecting transition region and coronal models}

The importance of the transition region between the chromospheric mass reservoir and the corona to the properties of the solar wind has been known for some years now (e.g., Lie-Svendsen et al, 2002; Hansteen and Velli, 2012, and reference cited therein). The coupling between these regions rests on the connection between the transition region pressure and the inward heat flux from the hot corona. In summary a high transition region pressure implies a dense corona, leading to high mass flux slow wind. Conversely, for the same energy input in the corona, a low transition region pressures lead to small mass flux but fast winds. As shown by Hansteen and Leer (1995) the density and temperature cannot be set independently in advance, but are indeed set by consistent solutions of the solar wind outflow that include some model of the chromosphere and transition region. This coupling can be understood by considering the energy transport between the chromosphere, corona and solar wind. Assume for a moment that energy is solely dissipated in the corona. Some of this energy must be transported down towards the chromosphere, there to be radiated away and to be used in heating and lifting particles as they flow up into the hotter corona. The remainder of the energy is 
expended in expelling particles from the gravitational pull of the Sun and accelerating them to solar wind speeds. (Radiative losses from the corona itself play a lesser role unless the corona is quite dense.) Using expressions for a collisiondominated heat flux, proportional to $T^{5 / 2} \nabla T$, Hansteen and Leer (1995) found that in the vast majority of models roughly $10 \%$ of the energy deposited went to the transition region corona, the remainder being available to accelerate the coronal plasma out of the Sun's gravitational field. In these solutions an excess heat flux is compensated by a greater transition region pressure until the density grows high enough to radiate all downflowing energy. Thus, to a good approximation

$$
P_{0} \approx C q_{\mathrm{e}}
$$

where $P_{0}$ is the transition region pressure, $C$ is a constant, and $q_{\mathrm{e}}$ the electron heat flux density. Furthermore, with only of order $10 \%$ of the injected mechanical energy flux, $F_{\mathrm{m} 0}$, going back into the transition region to be radiated away it is easy to show that the mass flux in the wind, $M$, is to a very good approximation proportional to $F_{\mathrm{m} 0}$ (Hansteen and Leer, 1995)

$$
M=\frac{\left(F_{\mathrm{m} 0}-F_{r, \mathrm{E}}\right)}{\frac{1}{2}\left(v_{\mathrm{g}}^{2}+v_{\mathrm{E}}^{2}\right)} .
$$

where $v_{\mathrm{g}}$ is the escape speed from the solar surface, $v_{\mathrm{E}}$ is the asymptotic solar wind speed and $F_{r, \mathrm{E}} \approx 0.1 F_{\mathrm{m} 0}$ represents the total radiative losses. The system functions as follows: with heating close to the transition region the percentage of energy flowing back to the transition region $q_{\mathrm{e}}$ is large (though still of order $\left.O(0.1) F_{\mathrm{m} 0}\right)$ leading to a dense low speed wind. Conversely, heating further from the Sun will result in smaller $q_{\mathrm{e}}$, a lower density corona, and therefore more energy per particle to accelerate a faster wind.

The analysis above is based on the assumption that the heat flux, and in particular the proton heat flux, can be described through classical heat conduction. The models described in Hansteen and Leer (1995) and Lie-Svendsen et al (2002) show that this may not always be the case: when heating goes into protons only, the proton temperature is predicted to be very high, of order $3-4 \mathrm{MK}$, casting into doubt whether the protons can be described as a collision-dominated gas. Using gyrotropic transport equations it was found that in super-radial coronal holes protons quickly became nearly collisionless and that the downward proton heat flux in the corona and transition region was much smaller than predicted by classical transport theory (Lie-Svendsen et al, 2002). In this case the connection between the corona and transition region was nearly broken, resulting in a very low-density corona and a very high asymptotic solar wind speed. This state of affairs is exacerbated in heating models where protons are heated perpendicular to the magnetic field and the mirror force causes most of the protons to mirror before they reach locations where energy must be deposited in order to maintain the mass flux or where they can transfer energy to the electron fluid which remain classical.

To achieve realistic solar wind mass fluxes and terminal velocities, especially for models of the SSW, the high-density solutions where the transition region density is proportional to the downward directed electron heat flux seem preferable. This type of solution is arrived at in cases where coronal heating is not solely going into the perpendicular component of the proton fluid, but rather also is heating 
the parallel directed protons or electrons, where the areal expansion factor is close to radial, or if the energy is also deposited directly into the transition region. Recent 3D numerical simulations constructed to model coronal heating through the braiding of the photospheric field (Hansteen et al, 2010) show that the latter possibility may indeed be the case and that heat deposition indeed is predicted in or close to the transition region, this in addition to any heat dissipation through Alfvén waves or similar that occurs at greater heights. Thus, for solar regions that are a mix of magnetically closed and open field configurations such as those proposed to be the site of SSW outflow, we expect a tight coupling between the properties of the wind and the transition region between the chromosphere and corona. When constructing models one should then either set the bottom boundary in the chromosphere to capture this process, or if a bottom boundary in the corona is desired, set the electron density as determined by the energy balance including both radiative losses and enthalpy flux into account (Lionello et al, 2001; Endeve and Leer, 2001).

\subsection{D MHD single fluid models}

The MHD approximation is appropriate for large-scale, low-frequency phenomena in magnetized plasmas such as the solar corona. Using the photospheric magnetic field as the primary driving boundary condition, single-fluid global MHD models can often reproduce the Sun's magnetic and emission properties during both quiet (e.g., Riley et al, 2001; Riley et al, 2006; Riley and Luhmann, 2012) and active (e.g., Riley et al, 2003, 2007, 2008) periods. In particular, most current formulations are based on some variant of the following set of viscous and resistive MHD equations:

$$
\begin{aligned}
\nabla \times \mathbf{B} & =\frac{4 \pi}{c} \mathbf{J} \\
\nabla \times \mathbf{E} & =-\frac{1}{c} \frac{\partial \mathbf{B}}{\partial t} \\
\mathbf{E}+\frac{\mathbf{v} \times \mathbf{B}}{c} & =\eta \mathbf{J} \\
\frac{\partial \rho}{\partial t}+\nabla \cdot(\rho \mathbf{v}) & =0 \\
\frac{1}{\gamma-1}\left(\frac{\partial T}{\partial t}+\mathbf{v} \cdot \nabla T\right) & =-T \nabla \cdot \mathbf{v}+\frac{m}{2 k \rho} S \\
\rho\left(\frac{\partial \mathbf{v}}{\partial t}+\mathbf{v} \cdot \nabla \mathbf{v}\right) & =\frac{1}{c} \mathbf{J} \times \mathbf{B}-\nabla\left(p+p_{w}\right)+\rho \mathbf{g}+\nabla \cdot(\nu \rho \nabla \mathbf{v}), \\
S & =\left(-\nabla \cdot \mathbf{q}-n_{e} n_{p} Q(T)+H_{\mathrm{ch}}\right),
\end{aligned}
$$

where $\mathbf{B}$ is the magnetic field, $\mathbf{J}$ is the electric current density, $\mathbf{E}$ is the electric field, $\rho, \mathbf{v}, p$, and $T$ are the plasma mass density, velocity, pressure, and temperature, $\mathbf{g}=-g_{0} R_{\odot}^{2} \hat{\mathbf{r}} / r^{2}$ is the gravitational acceleration, $\eta$ the resistivity, and $\nu$ is the kinematic viscosity. Equation (7) contains the radiation loss function $Q(T)$ as in Athay (1986), $n_{e}$ and $n_{p}$ are the electron and proton number density (which are equal for a hydrogen plasma), $\gamma=5 / 3$ is the polytropic index, $H_{\mathrm{ch}}$ is the coronal heating term, and $\mathbf{q}$ is the heat flux. 

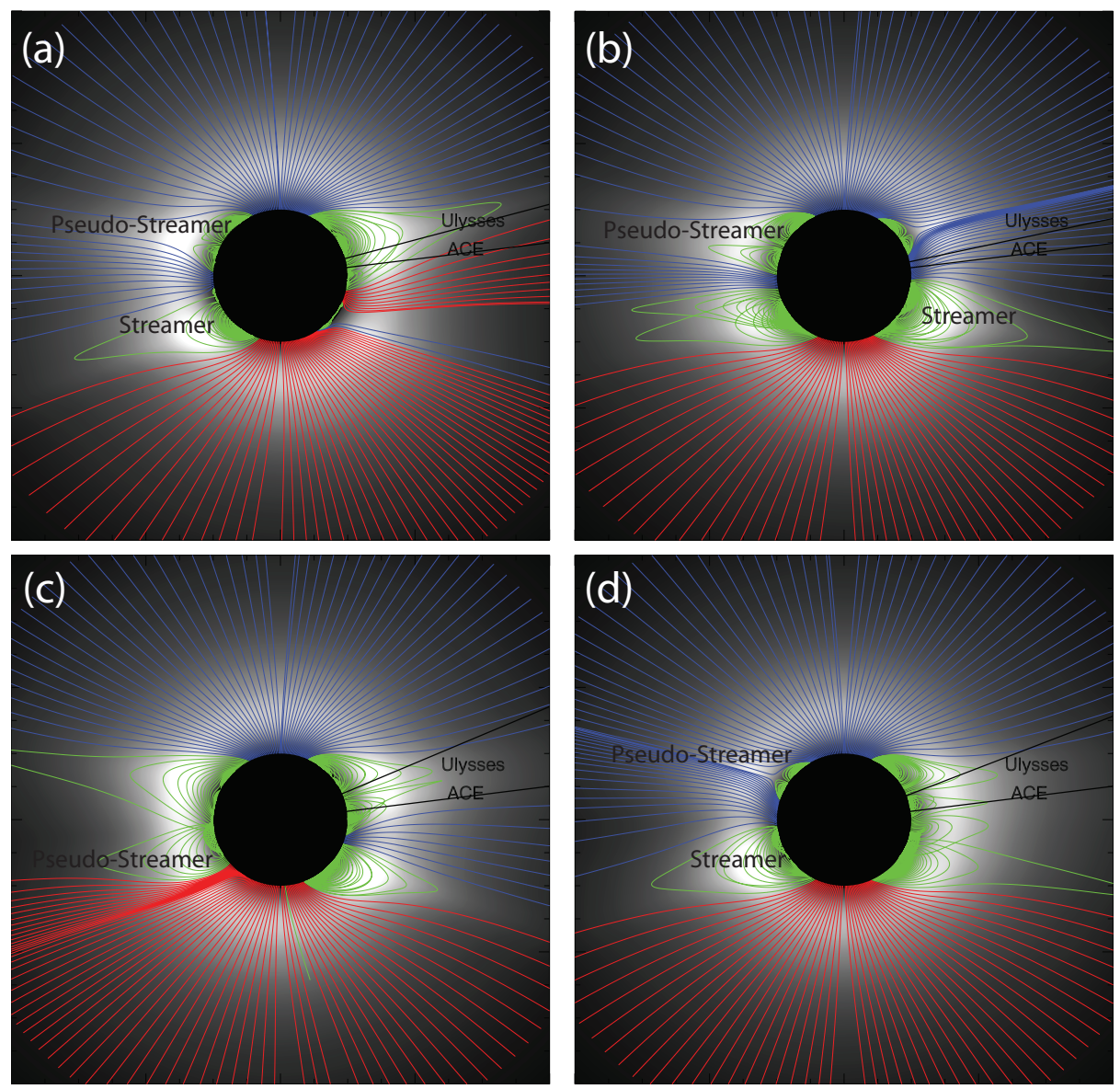

Fig. 12 A selection of meridional slices from a global MHD simulation of CR 2060, which occurred between 14 August 2007 and 10 September 2007. Grey-scale images are simulated polarized brightness $(\mathrm{pB})$ images and the colored lines are magnetic field lines drawn from equally spaced points in latitude on the solar surface. The field lines have been color-coded so that blue/red lines are field lines that open into the heliosphere and are inwardly/outwardly directed, while green field lines connect back to the Sun at both ends, i.e., they are closed field lines. From Riley and Luhmann (2012).

Current global MHD models rely on several techniques to reproduce the observed values and variability of the solar wind. The simplest is the so-called polytropic approximation, where the energy equation is replaced by a simple polytropic relationship between density and pressure (i.e., $S=0$ ), and $\gamma$ is set to 1.05 to mimic the near-isothermal nature of the corona (e.g., Linker et al, 1990; Riley et al, 2001). Whilst this simplifies the simulations tremendously, it results in plasma properties, or at least their variability, that do not match observations very well. Alternatively, a semi-empirical, variable polytropic index is used with somewhat better results (e.g., Cohen et al, 2007).

In the more sophisticated "thermodynamic" approximation, energy transport processes are included more explicitly (Lionello et al, 2009). Coronal heating is treated 


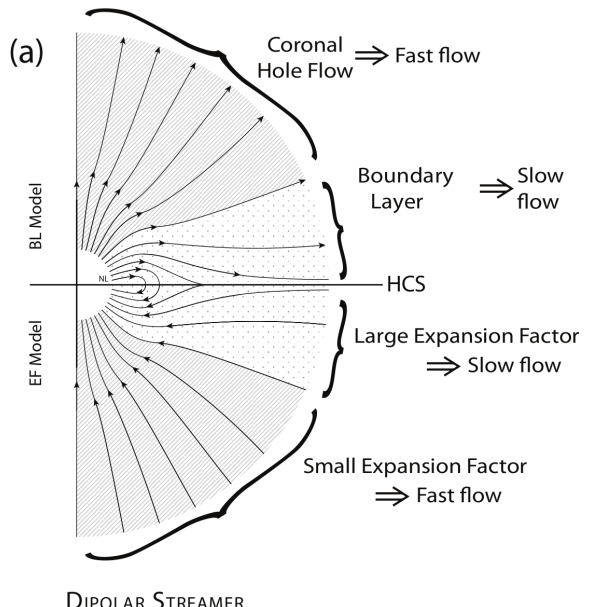

Dipolar Streamer

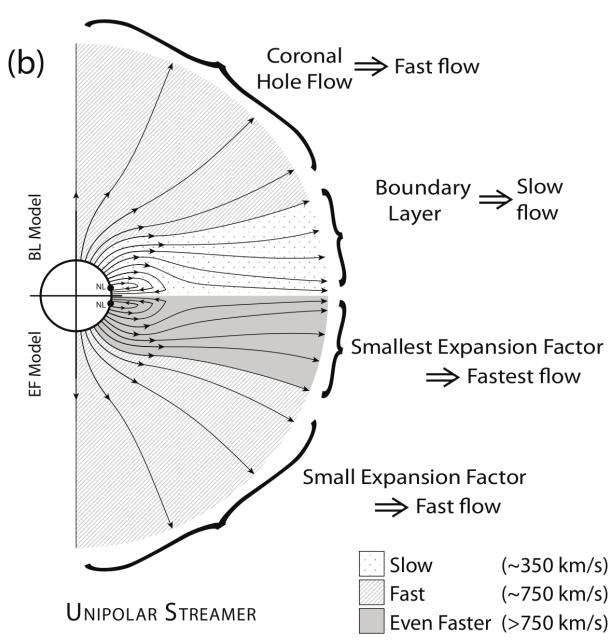

Fig. 13 An illustration of the salient features of the expansion factor and boundary layer models for: (a) a dipolar streamer and (b) a unipolar streamer. From Riley and Luhmann (2012).

in an ad hoc manner; however, these semi-empirical formulations are designed to mimic the types of processes we believe are responsible for heating the corona. Moreover, detailed comparisons with EUV and X-ray emission measurements suggest that they have captured the essential properties of the heating profiles, in spite of them circumventing the underlying physical processes.

Most recently, global MHD models have attempted to incorporate self-consistent theories of coronal heating and solar wind acceleration using waves and turbulence (e.g., Lionello et al, 2013; van der Holst et al, 2014). Such approaches can, in principle, be used to support, refute, or constrain theories for the origin of the SSW on open field lines based on turbulent fluctuations (Cranmer et al, 2014).

Interestingly, global MHD models have only occasionally been used to assess the contribution to the SSW from interchange reconnection (e.g., Lionello et al, 2005), the other leading idea for the origin of the SSW. In part, this is because it has proven difficult to develop reliable time-dependent synoptic (or, more strictly, synchronic) maps. Additionally, such time-dependent calculations can lead to numerical issues. Recently, with the development of flux-evolved, time-dependent synchronic maps, such as those produced by the Air Force data assimilative photospheric flux transport (ADAPT) model (Arge et al, 2010), we have begun to develop time-dependent model solutions. We anticipate that these results will provide a unique way to differentiate between wave-turbulence models and interchange reconnection models for the generation of the SSW.

The declining phase of cycle 23, leading to the prolonged solar minimum of 2008/2009 has provided a unique opportunity to investigate the origin of the SSW. In particular, the extended presence of pseudo-streamers has allowed us to distinguish between two leading theories for the origin of the SSW (Riley and Luhmann, 2012); the expansion-factor model, which predicts fast solar wind at pseudo- 
streamers due to the small value of the expansion factor, and "boundary layer" models that predict slow solar wind in these regions. Observations consistently show that wind emanating from pseudo-streamers is not fast. For illustration, Fig. 12 shows four views of the corona during CR 2060, which occurred between 14 August 2007 and 10 September 2007.

The underlying physical origins of these structures are drawn in Fig. 13, which shows the main features of the expansion factor model (presumably driven by waves/turbulence alone) and boundary layer models ("interchange reconnection" being the most promising candidate mechanism). For helmet (dipolar) streamers, both models predict SSW on either side of the HCS. The width of the slow-flow band is determined in the boundary layer model by the details of where the reconnection is taking place, or the scale over which instability is occurring, but is presumably limited to some distance away from the closed loops. The boundary of the slow-flow band in the expansion factor model is determined by an interesting property of the time-independent Parker equations, namely, that for rapidly expanding flux tubes, there may be more than one location for the critical point (Cuperman et al, 1990), the most stable of which is that one furthest from the Sun (Cranmer et al, 2007).

\section{$3.32 \mathrm{D}$ and 3D MHD multi-fluid models}

Since the launch of SOHO, coronal streamers have been imaged by UVCS in multiple emission lines as discussed in details on Section 2.1.2. These observations cannot be adequately modeled with a single fluid MHD model. Therefore, a selfconsistent $2.5 \mathrm{D}$ multi-fluid model of coronal streamers was developed (Ofman, 2000, 2004a; Li et al, 2006; Ofman et al, 2011, 2013). Another approach was to use the flux tube geometry obtained from 2D MHD model of streamers to calculate the minor ion $\left(\mathrm{O}^{5+}\right)$ outflow (Chen and $\mathrm{Li}, 2004$; Chen et al, 2004). However, this approach is not well applicable to models with $\mathrm{He}^{++}$ions due to the significant effect of these ions on the solar wind outflow. One of the first comparisons between UVCS observations and a 2.5D multi-fluid model has been published by Ofman (2004a) and in more details by Abbo et al (2010b); Ofman et al (2011, 2013). Recently, the multi-fluid model was extended to full 3D and applied to the tilted dipole solar magnetic field (Ofman et al, 2015).

The multi-fluid equations are obtained by approximating the electrons, protons, and other ions as collisionally, and electro-magnetically coupled fluids of charges (with overall quasi-neutrality) (Braginskii, 1965; Li et al, 1997; Ofman, 2004b) that are used to model the SSW in coronal streamers. In the derivation the electron inertia is neglected, leading to an expression for the electric field - the generalized Ohm's law. With the standard notation the normalized equations can be written as

$$
\frac{\partial n_{k}}{\partial t}=-\nabla \cdot\left(n_{k} \mathbf{V}_{k}\right)
$$




$$
\begin{aligned}
n_{k}\left[\frac{\partial \mathbf{V}_{k}}{\partial t}+\left(\mathbf{V}_{k} \cdot \nabla\right) \mathbf{V}_{k}\right]= & -E u_{k} \nabla p_{k}-E u_{e} \frac{Z_{k} n_{k}}{A_{k} n_{e}} \nabla p_{e}-\frac{n_{k}}{F_{r} r^{2}} \mathbf{e}_{r}+ \\
& +\Omega_{k} n_{k}\left(\mathbf{V}_{k}-\mathbf{V}_{e}\right) \times \mathbf{B}+\mathbf{F}_{v}+n_{k} \mathbf{F}_{k, \text { coul }}, \\
\frac{\partial \mathbf{B}}{\partial t}= & \nabla \times\left(\mathbf{V}_{e} \times \mathbf{B}\right)+\frac{1}{S} \nabla^{2} \mathbf{B} \\
\mathbf{V}_{e}= & \frac{1}{n_{e}}\left(n_{p} \mathbf{V}_{p}+Z_{i} n_{i} \mathbf{V}_{i}-b \nabla \times \mathbf{B}\right), \\
\frac{\partial T_{k}}{\partial t}= & -\left(\gamma_{k}-1\right) T_{k} \nabla \cdot \mathbf{V}_{k}-\mathbf{V}_{k} \cdot \nabla T_{k}+ \\
& +C_{k j l}+\left(\gamma_{k}-1\right)\left(S_{k}-\delta_{k, e} S_{r, e}\right) .
\end{aligned}
$$

In the above equations the index $k$ indicates ion species, and the normalization parameters are the Lundquist number $S$, the Euler numbers $E u_{e, p}=\left(k_{b} T_{0, e, p} / m_{p}\right) V_{A}^{2}$, $E u_{i}=\left(k_{b} T_{0, i} / m_{i}\right) V_{A}^{2}$, the Froude number $F_{r}=V_{A}^{2} R_{\odot} / G M_{\odot}$ where $G$ is the universal gravitational constant and $M_{\odot}$ is the solar mass, $b=c B_{0} /\left(4 \pi e n_{e 0} R_{\odot} V_{A}\right)$, and the Bolzmann constant $k_{b}$. The Alfvén speed $V_{A}$ is defined by $B_{0} / \sqrt{4 \pi m_{p} n_{e 0}}$. The details of the Coulomb friction terms between the species in the momentum equation $\mathbf{F}_{k, \text { coul }}$, and the energy exchange terms $C_{k j l}$ can be found in Ofman (2004b). Furthermore, $\Omega_{k}$ is the ion gyro-frequency and is determined by the parameters $A_{k}$ (mass number) and $Z_{k}$ (charge number). The normalizations of the magnetic field $B_{0}$, electron density $n_{e 0}$, and temperatures $T_{k}$ are used to calculate the above parameters. In the energy equations (12) the $S_{k}$ terms represent the heating and cooling terms in the coronal plasma, which may include wave heating and heat conductions. The radiative cooling term $S_{r, e}$ only enters in the electron energy equation. A variation on the three-fluid equations applicable to $1 D$ models is available at Li et al (2006).

Below, we review some of the results of modeling the SSW obtained by solving the 2.5D and 3D multifluid equations with electrons, protons, and other ions.

Ofman (2000) and Ofman et al (2011) solved Equations (8) - (12) in 2.5D (i.e., with the assumption of azimuthal symmetry) with $\mathrm{O}^{5+}$ as the third species, and found good agreement with UVCS spectroscopic observations of quiescent streamers at solar minimum. While Ofman (2000) used a single quadrant of a quadrupole field with isothermal coronal plasma to initiate the streamer modeling, Ofman et al (2011) extended the model by using the dipole field for the corona to initiate the modeling of an equatorial streamer belt, which approximates the conditions at solar minimum, and an exponential empirical heating term for the $\mathrm{O}^{5+}$ with the parameters chosen to match the kinetic temperature from UVCS spectroscopic observations. It was found by detailed comparison with observations that the results of the three-fluid model are in good agreement with the observed streamer density, temperature, and velocity structure of the SSW close to the Sun as seen from UVCS spectroscopic data. Moreover, the calculated synthetic emission images based on the model output are in good agreement with the observed emission of Ly $\alpha$ and O VI.

The dynamics and the effects of $\mathrm{He}^{++}$ions $(\alpha$ particles) on the SSW in a three-fluid 2.5D model were first considered by Ofman (2004a). Also incorporating $\alpha$ 's into a (quasi-)steady, 2.5D, three-fluid model of the solar corona and solar wind, $\mathrm{Li}$ et al (2006) provided a detailed examination 
on how $\alpha$ particles affect the solar wind parameters as well as the coronal magnetic field configuration. For simplicity, protons and $\alpha$ particles are heated by an empirical energy flux, whereas electrons are heated only by thermal conductive flux and their Coulomb coupling with ions. Fig. 14 presents the solar wind parameters hence derived as a function of heliocentric distance from the coronal base out to the orbit of the Earth. Two flow tubes, anchored at colatitudes $2^{\circ}$ (left column) and $57.5^{\circ}$ (right) on the Sun, are followed to show the solutions representative of the modeled fast and slow winds, respectively. A comparison is made with a number of observations, as represented by the error bars in Figs. 14a and 14c as well as the diamonds in Fig. 14d. The temperature ratios of the protons and $\alpha$ 's and the expansion factor $f$ are shown in Figs. 14e-f. Furthermore, the model results are also compared with some measurements made in situ. Such comparisons yield that for realistic proton $-\alpha$ particle speed difference at 1 AU to be achieved, $\alpha$ particles need to be heated preferentially over protons in the inner corona. In addition, varying the $\alpha$ particle abundance at the base from 0.015 to 0.15 , some further computations by $\mathrm{Li}$ et al (2006) indicated that $\alpha$ 's play a negligible role in determining the magnetic field distribution. However, due to the substantially larger mass relative to protons, $\alpha$ particles can heavily impact the solar wind parameters. Actually they are also important in determining the thermal structures in streamers: a preferential heating of $\alpha$ particles can result in increased proton and electron temperatures (Ofman and Kramar, 2010). It should be noted that in both $\mathrm{Li}$ et al (2006) and Ofman and Kramar (2010), a crossstreamer structure similar to $\mathrm{O}^{5+}$ is also seen for the $\alpha$ particles, as far as their densities and flow speeds are concerned.

The results of the $2.5 \mathrm{D}$ multifluid model of a quiescent streamer with $\mathrm{Mg}^{9+}$ as the third ion obtained by Ofman et al (2013) are shown in Fig. 15. The density of $\mathrm{Mg}^{9+}$ is shown on a logarithmic scale in Fig. 15a, and the proton density on a logarithmic scale is shown in Fig. 15b. It is evident that the $\mathrm{Mg}^{9+}$ is depleted in the center of the streamer, and in the stalk compared to the exterior of the streamer. This is due to the gravitational settling of the heavy ions in the core of the streamer. Outside the streamer core the Coulomb friction with the outflowing protons and electrons leads to increased $\mathrm{Mg}^{9+}$ density in the SSW. Protons show an opposite behavior with their densities enhanced in the core of the streamer, due to confinement and the Lorentz force that arises from pressure balance between the core and the streamers' exterior.

Recently, Ofman et al (2015) used the above multi-fluid equations (8)-(12) and extended the model to full 3D, allowing one to study the SSW in 3D tilted dipole configuration of the solar magnetic field that may be applicable during solar activity minimum conditions, as well as more realistic magnetogram-based field. In Fig. 16 the 3D magnetic structure of the tilted dipole streamer belt obtained by Ofman et al (2015) is shown. The field lines are stretched by the expanding SSW and form the typical helmet streamer configuration. The right panels show the cuts of proton and $\mathrm{O}^{5+}$ densities in the $\phi-\theta$ plane at $r=1.5 R_{\odot}$. The inverted density structure of $\mathrm{O}^{5+}$ with respect to protons is evident as well in $3 \mathrm{D}$ configuration, with additional detail due to the tilt. It is evident that $3 \mathrm{D}$ modeling is important 

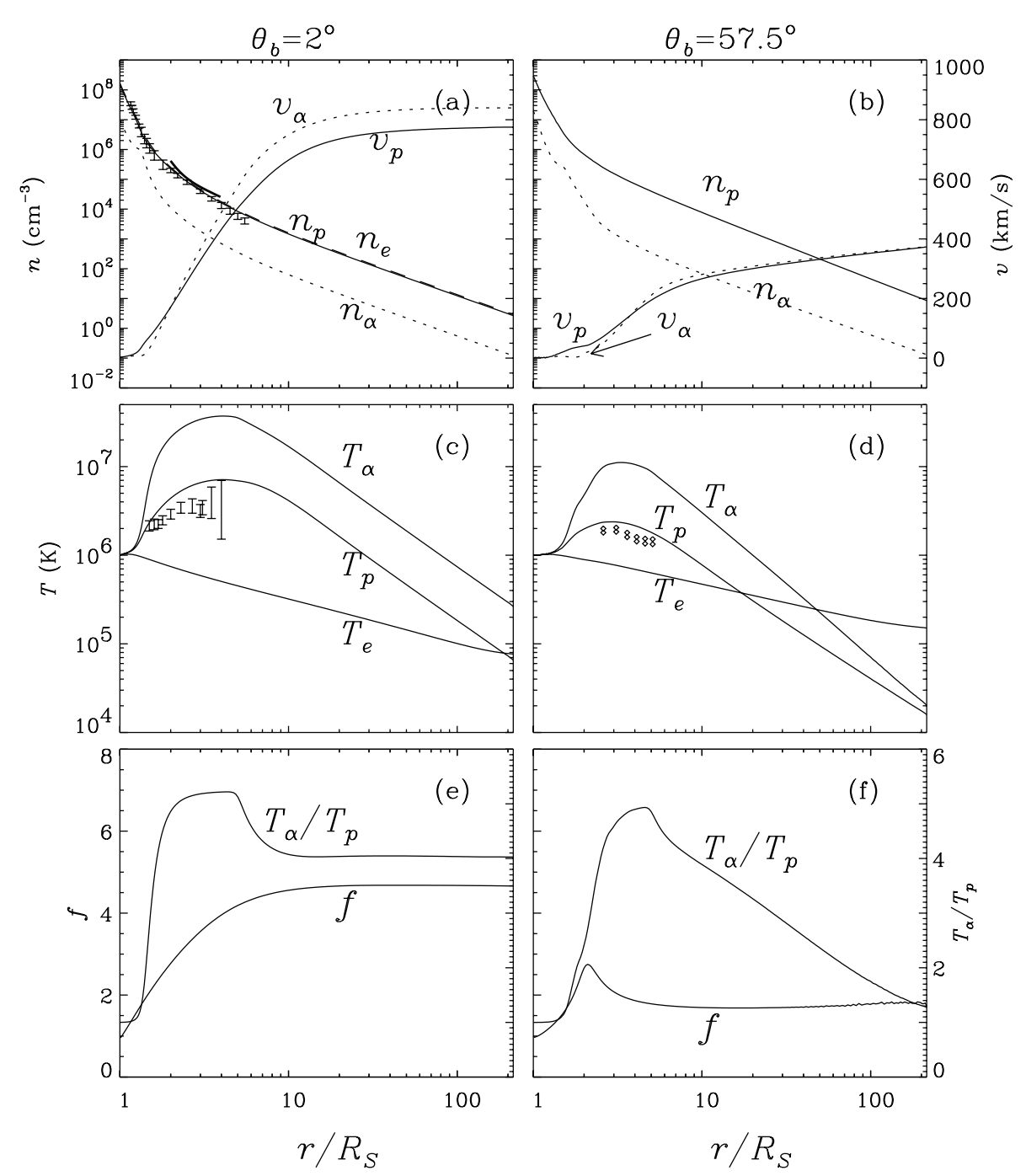

Fig. 14 The radial profiles of the proton and $\mathrm{He}^{++}$ion ( $\alpha$ particles) fluid variables at two flux tubes $\left(2^{\circ}\right.$ near the polar region and $57.5^{\circ}$ along the streamer edge) obtained with the 2.5D three-fluid model in an equatorial streamer belt. (a) and (b) the densities and velocities, (c) and (d) the temperatures of the ions and electrons, (e) and (f) the temperature ratios and the expansion factors of the two flux tubes. In (c) $\mathrm{H}$ Ly $\alpha$ line width measurements are plotted with error bars (Kohl et al, 1998), and in (d) the limits from similar measurements in streamers are shown (Frazin et al, 2003). In this three-fluid model the ion species are heated by an empirical energy flux, whereas the electrons receive no external heating apart from electron heat flux and energy exchange due to Coulomb coupling with the ions. Adapted from Li et al (2006). 

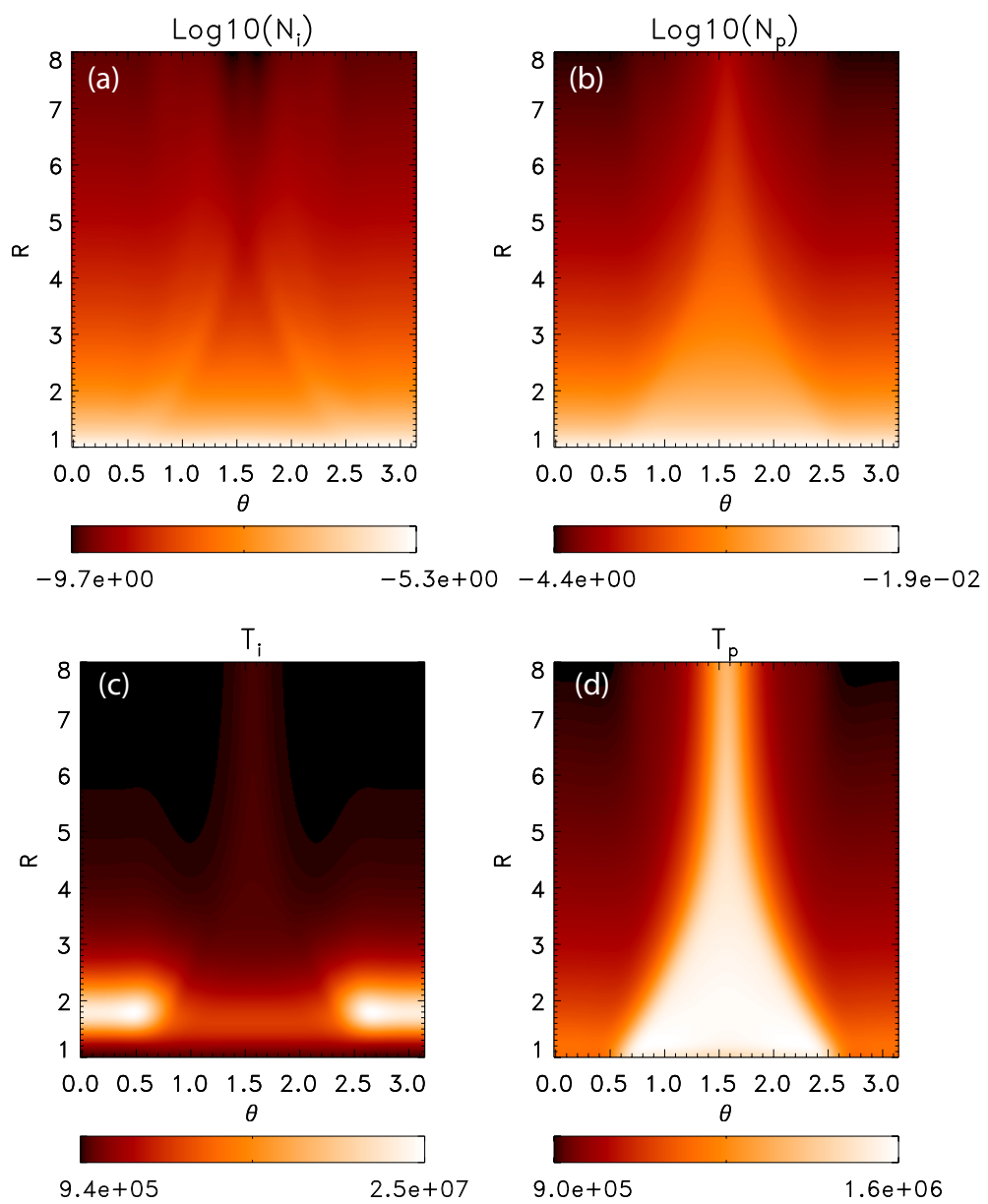

Fig. 15 The results of the $2.5 \mathrm{D}$ multifluid model with $\mathrm{Mg}^{9+}$ of a streamer at the (quasi) steady state. (a) Normalized $\mathrm{Mg}^{9+}$ ion density, and (b) normalized proton density $\left(\log _{10}\right.$ scale). The corresponding temperatures in Kelvin of (c) $\mathrm{Mg}^{9+}$ ions, and (d) protons. Adapted from Ofman et al (2013).

in order to account for line-of-sight effects even in a tilted dipole configuration at solar minimum. The $3 \mathrm{D}$ modeling is crucial for understanding multiple streamer images in various ion emission lines during solar maximum, as well as for addressing the physical effects that arise from azimuthal derivatives of the variables that are neglected in the $2.5 \mathrm{D}$ model.

We note that important extension to the multifluid model can be made by incorporating species temperature anisotropy. Spectroscopic measurements made by SOHO/UVCS have demonstrated that at least for the O VI ions, the perpendicular temperature tends to considerably exceed the parallel one beyond a fraction of a solar radius. This is true not only for the nascent fast solar wind above coronal holes (Cranmer et al, 1999a, 2008), but also for the near-Sun slow wind immediately adjacent to coronal streamers (see Section2.1.2). To address the 

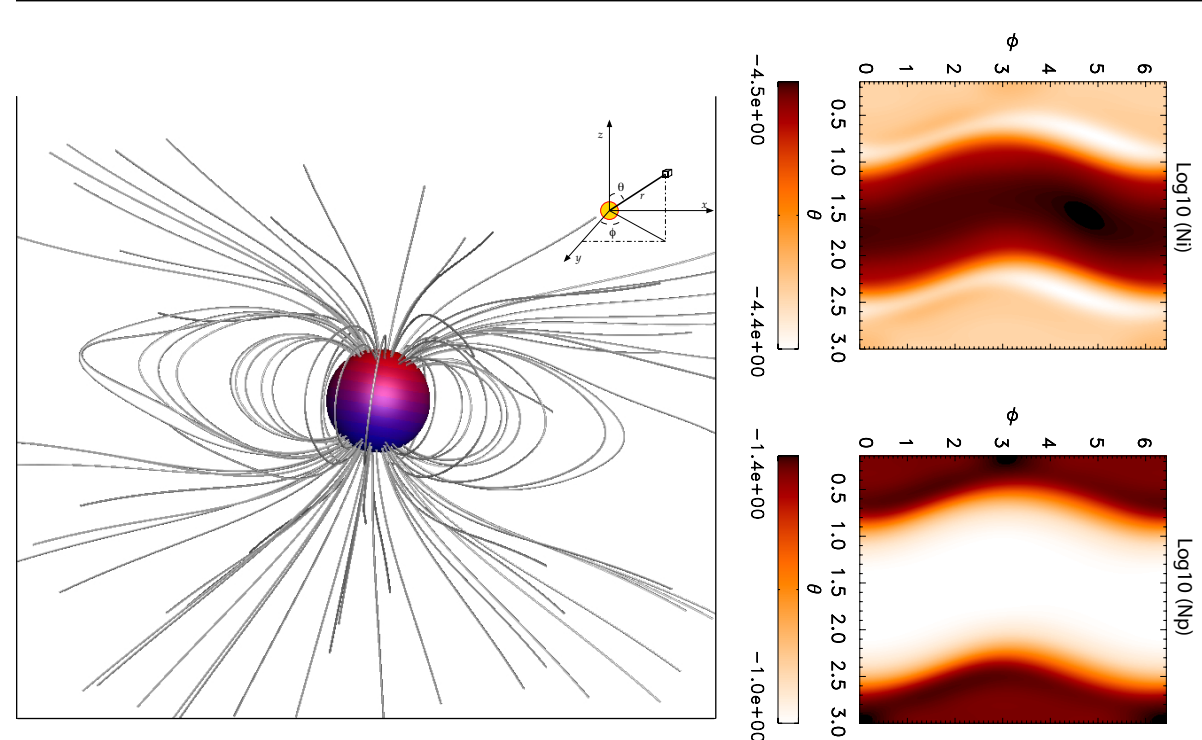

Fig. 16 Left panel: the 3D magnetic structure of the coronal field in the steady state solution using the 3D three-fluid model. The formation of the tilted streamer belt configuration is evident. Right panel: The proton and $\mathrm{O}^{5+}$ ion density in the $\phi-\theta$ plane at $r=1.5 R_{\odot}$ of the streamer belt. Adapted from Ofman et al (2015).

ion temperature anisotropy in a multi-fluid framework involves modifying both the mathematical and physical ingredients of the current model. Mathematically, one may start with the classic 16-moment transport equations (e.g., Barakat and Schunk, 1982) and treat the ion energetics in the perpendicular and parallel degrees of freedom on an equal footing, resulting in separate equations governing the parallel and perpendicular ion temperatures. In addition, the pressure gradient force in the ion momentum equations will appear as the divergence of a pressure tensor. Physically, one may proceed with the same empirical fashion as in Ofman (2004a); Li et al (2006) by replacing the source term $S_{k}$ in Eq. (12) with separate ad hoc parallel and perpendicular heating functions. Alternatively, for describing the heating terms one may invoke mechanisms based either on ion-cyclotronresonance (see the review by Hollweg and Isenberg, 2002) or on a perpendicular turbulent cascade (Chandran et al, 2011, and references therein). However, while both mechanisms have been incorporated in multi-dimensional global models to account for proton temperature anisotropies ( $\mathrm{Li}$ et al, 2004; van der Holst et al, 2014), a 2.5D or fully 3D model has yet to be developed to address the anisotropy of a second ion species. This advance seems imperative as far as the $\mathrm{O}^{5+}$ ions are concerned. One must bear in mind, however, that any fluid description cannot model the underlying processes that lead to temperature anisotropy from first principles. These kinetic processes require at least hybrid modeling approach, where the protons and other ions, such as $\mathrm{He}^{++}$and $\mathrm{O}^{5+}$ are modeled as particles, while the electrons can be still modeled as a background fluid in the $2.5 \mathrm{D}$ expanding solar wind model (see, recently Ofman et al, 2014; Maneva et al, 2015; Ozak et al, 2015). Although the primary focus of these hybrid models was the fast 
solar wind - similar kinetic processes are likely important in the slow wind open field acceleration regions.

\section{Which are the main SSW sources and their formation mechanisms?}

Currently, it is believed that the formation and sources of the SSW are associated with several scenarios that combine the physical effects of magnetic topology, source regions in the corona, and acceleration/heating mechanisms. In general we can classify the SSW into three scenarios. The first scenario (SSW1) is that the slow wind is governed by the same acceleration and heating process as the fast solar wind where it is associated with open magnetic field regions that have larger expansion factor and/or field line curvature (as compared to the fast wind regions) resulting in slower solar wind velocity (e.g., Wang and Sheeley, 1990; Li et al, 2011). This can be understood schematically by the fact that the input momentum and energy flux in the low corona spreads rapidly over increasing area of a magnetic flux tube, resulting in lower wind speed. A wave-driven wind model that has been applied extensively, mostly to fast solar wind models can also drive the slow wind accounting for the variability and the effects of expansion on wave acceleration were investigated in the past (e.g., Ofman and Davila, 1998), while kinetic (resonant) waves can provide the differential heating of ions often observed in the solar wind plasma (see the review, Ofman, 2010). In open field regions the acceleration of the wind can occur through direct momentum transfer from low frequency large amplitude MHD waves or shocks (e.g., Ofman and Davila, 1998; Nakariakov et al, 2000; Suzuki, 2004).

In the following scenarios the source of momentum and energy that produces the SSW is the magnetic reconnection and the Lorentz force produced by the resulting currents and fields. The second scenario (SSW2) is associated with interchange reconnection between open field in the coronal hole with nearby closed coronal loops. This process may release the closed-field plasma into the open field/solar wind (e.g., Fisk and Schwadron, 2001), or with complex coronal magnetic field topology with inversed polarity (separatrics) that favors magnetic reconnection and channel the accelerated plasma that ultimately is released into the open field lines expanding into the heliosphere (e.g., Antiochos et al, 2011; Titov et al, 2011). The third scenario (SSW3) is related to the weak magnetic field at the streamer cusp which makes it possible for the material in the underlying closed-field regions to be released into the solar wind through magnetic reconnection, diffusion or thermal instabilities (e.g., Suess et al, 1999; Einaudi et al, 1999). It should be noted that waves and turbulence can heat the plasma in closed magnetic field configurations as well, and this can provide the source of energy through thermal pressure that accelerates the plasma in closed field as it is injected into open field regions through the above reconnection scenarios.

For illustrative purposes we show schematically the various SSW source regions and formation mechanisms in Fig. 17 by combining the composite image of the 2006 March 29 eclipse, edge-enhanced SOHO/LASCO image adapted from Fig. 4 of Wang et al (2007a) with EIT EUV image of the corona, and inserts that summarize the different hypotheses of the SSW formation at the various sources: 
(a) expansion factor/curvature of the magnetic topology adjacent to the helmet streamer, (b) interchange reconnection between open field and closed loop in a helmet streamer, (c) interchange reconnection between open field and closed loop in a pseudo-streamer, (d) complex magnetic structure with quasi-separatrix layers creating a favorable environment for reconnection (S-web model), (e) releasing of closed loop material as plasma blobs at the cusp of a helmet streamer, and (f) that points to turbulence/waves solar wind heating and acceleration mechanism, that is important in open field structures. Case (a) is related to the above SSW1 scenario, while (b), (c), (d) to SSW2 scenario; case (e) refers to SSW3; case (f) refers to SSW1 scenario that is likely important in open field regions (with high expansion factor for the SSW). In principle, the wave and turbulent heating can energize the plasma in closed fields as well - as has been studied for decades, and this energy can be channeled into open field as a result of interchange reconnection, providing additional source of SSW acceleration.

In the following, the general hypotheses for the SSW formation mechanisms, which are expansion factor model (see section 4.1), interchange reconnection model (see section 4.2), S-web model (see section 4.3) and magnetic reconnection at the cusp (see section 4.4) are described in more details.

\subsection{Expansion factor model}

The simplest hypothesis is that the bulk of the solar wind comes from long-lived open field regions or coronal holes (e.g., Suess, 1979; Leer et al, 1982; Withbroe, 1988; Cranmer, 2009). For example, using OSO-7 Goddard Space Flight Center X-Ray and EUV Spectroheliograph data Bell and Noci (1976)

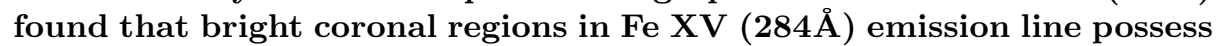
magnetic fields of closed configuration, thus reducing particle escape, while coronal holes possess open magnetic field lines favorable to particle escape or enhanced outflow of the solar wind. In this type of model, variations in the properties of the solar wind are determined by differences in the flow geometry or in the deposition of energy and momentum along open field lines. One of the key parameters is $f_{\mathrm{ss}}$, defined as the factor by which a given flux tube expands in solid angle between the coronal base and the source surface ( $\left.r=R_{\mathrm{Ss}} \sim 2.5 R_{\odot}\right)$, where the field is assumed to become radial.

Since the Skylab era, it has been recognized that the areal cross sections of coronal holes diverge much faster than radially (Munro and Jackson, 1977). This has led to a widespread misconception that high wind speeds are correlated with rapid areal expansion (e.g., Kopp and Holzer, 1976; Kovalenko, 1981). In fact, however, comparisons between photospheric field extrapolations and solar wind measurements at $1 \mathrm{AU}$ show the opposite to be the case (Levine et al, 1977; Wang and Sheeley, 1990): the faster a coronal flux tube expands, the slower the asymptotic wind speed $v$. In general, low-speed wind originates from just inside the boundaries of coronal holes, where $f_{\mathrm{ss}}$ has its largest values, whereas very fast wind is associated with the interiors of large holes, where $f_{\mathrm{ss}}$ is relatively small (but still greater than unity). Major sources of the slow wind during the solar cycle include the edges of the polar coronal holes and - especially near sunspot maximum, when the polar 


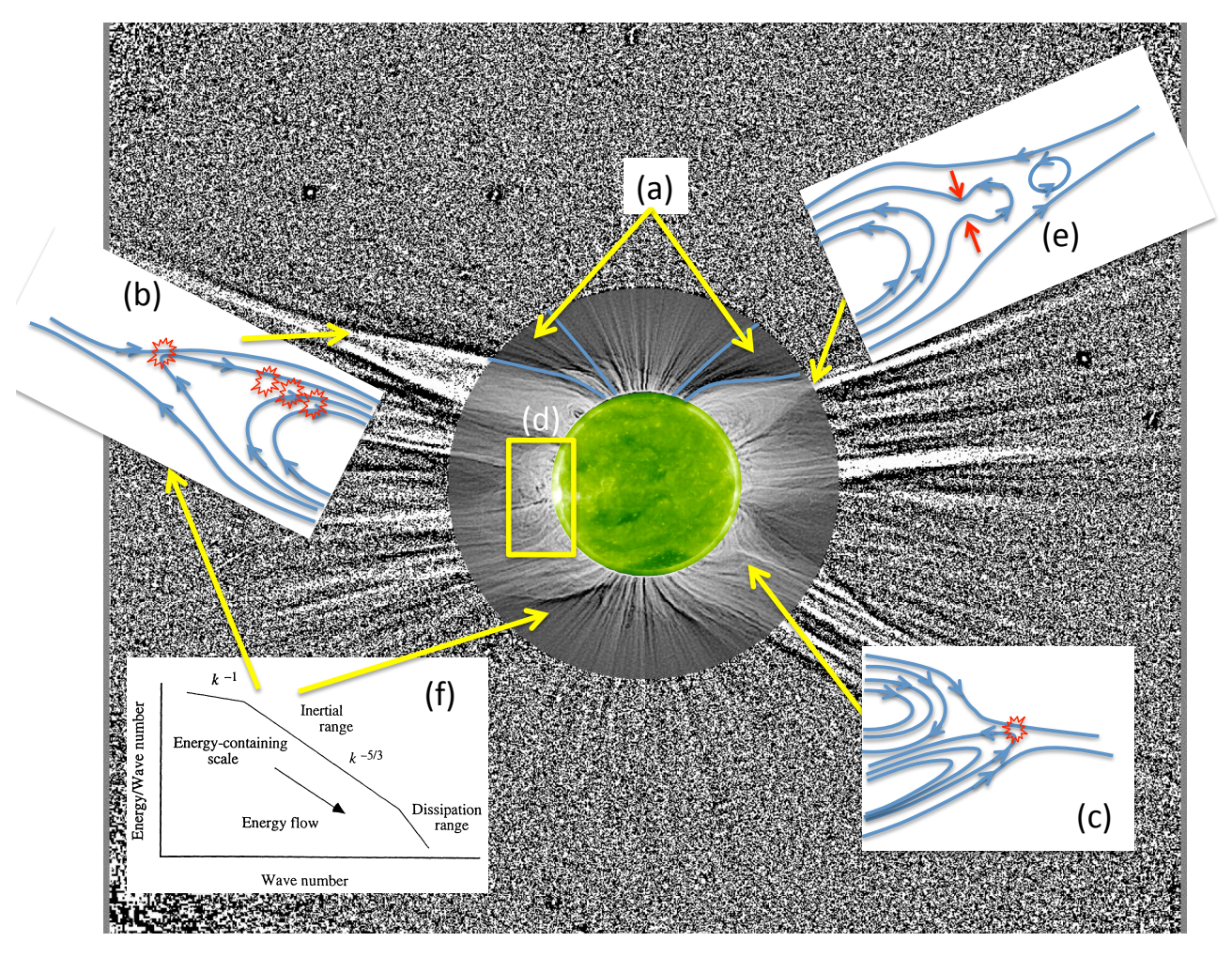

Fig. 17 An illustration of the scenarios for SSW formation at the various sources. The background image shows the white light 2006 March 29 eclipse image of the corona, edge-enhanced SOHO/LASCO image adapted from Fig. 4 of Wang et al (2007a), and an EUV SOHO/EIT corona. The sources/formation mechanisms are (a) expansion factor/curvature of the magnetic topology adjacent to the helmet streamer, (b) interchange reconnection between open field and closed loop in a helmet streamer, (c) interchange reconnection between open field and closed loop in a pseudo-streamer, (d) complex magnetic structure with quasi-separatrix layers creating a favorable environment for reconnection (S-web model), (e) releasing of closed loop material as plasma blobs at the cusp of a helmet streamer and (f) that points to turbulence/waves solar wind heating and acceleration mechanism, that is important in open field structures.

holes disappear-the small open-field regions/holes that form at the peripheries of active regions.

The physical basis for the inverse correlation between wind speed and the flux tube expansion factor is easily understood, if one assumes that the heating rate in coronal holes depends on the local magnetic field strength (see Wang et al, 2009b). When the field strength falls off rapidly with height (i.e., when $f_{\mathrm{ss}}$ is large), the heating will necessarily be concentrated near the coronal base. In that case, most of the energy will be conducted downward into the transition region, increasing the mass and enthalpy flux, while reducing the energy available per proton and thus the asymptotic wind speed. On the other hand, when the magnetic field falls off slowly with height, the energy will be deposited over a large distance extending 
out toward the sonic point, and more of it will go into accelerating the wind and less into increasing the mass flux (see the discussion of Leer and Holzer, 1980).

The tendency for slow wind to have higher oxygen freeze-in temperatures than fast wind can be explained similarly. Because of their low collisional ionization rates, the oxygen charge states decouple from one another just above the coronal base. Along rapidly diverging flux tubes, the temperature maximum occurs close to the coronal base, because that is where most of the energy is deposited; thus slow wind will be characterized by high $\mathbf{O}^{7+} / \mathbf{O}^{6+}$ ratios. These ratios will be especially enhanced in active region holes, where the field is very strong at low heights and the temperatures are correspondingly high. Depositing the energy at low heights may also cause easily ionized elements to be dragged out from the chromosphere by evaporating protons, producing a low-FIP enhancement in the slow wind.

By combining near-Earth solar wind measurements with the conservation of mass and energy along a flux tube, it can be shown that the mass and energy flux densities at the coronal base increase almost linearly with the footpoint field strength $B_{0}$ (see Wang, 2010). This empirical result has a number of important implications. First, it supports the assumption that the magnetic field is the basic source of the heating in coronal holes. Second, because $B_{0}$ may vary by more than two orders of magnitude, the heating rate in coronal holes varies over a very wide range, with active region holes being characterized by much stronger heating and larger mass-flux densities than the polar holes. Third, the variation of the mass flux density at $1 \mathrm{AU}$ remains very modest because the mass flux density at the Sun and the net flux tube expansion both increase almost linearly with $B_{0}$, so that the two effects offset each other. The expansion factor model thus provides a mechanism for regulating the solar wind mass flux (see Fig. 18). It is unclear how such regulation could be achieved if the bulk of the slow wind were generated by (e.g.) the transient opening of coronal loops.

A characteristic property of the flux tube expansion factor is that it increases rapidly near the edges of open field regions, but varies slowly otherwise, remaining roughly constant in the interiors of large coronal holes. This property may at least partially explain the observed tendency for fast wind to be far steadier than slow wind. In addition, the large holes that are the source of high-speed streams tend to have rather uniform and slowly evolving footpoint fields, unlike the small activeregion holes that appear to be the source of much of the slow wind near sunspot maximum. It should also be emphasized that the slow wind has multiple sources, including streamer blobs, the heliospheric plasma sheet, slow CMEs, as well as coronal holes and their boundary regions.

Cranmer et al (2007) have argued that non-steady solar wind solutions exist even for completely time-independent flux tube geometry and energy/momentum input. In contrast, MHD simulations invariably find a well-behaved steady-state solution for the wind irrespective of the expansion factor and, hence, of the wind speed (e.g., van der Holst et al, 2014).

The great majority of coronal flux tubes undergo monotonic expansion out to the source surface $r=R_{\mathrm{ss}}$, where the expansion factor attains its maximum value. An important exception occurs in the vicinity of pseudo-streamers, which separate 

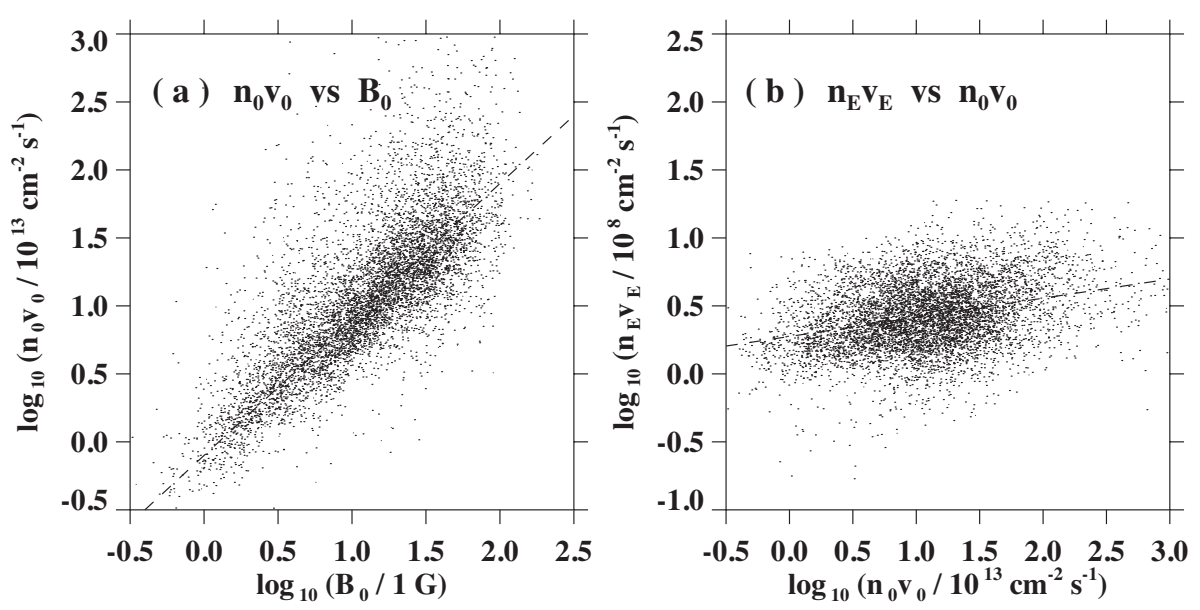

Fig. 18 Scatter plots of (a) proton flux density at the coronal base $\left(\log _{10}\left(n_{0} v_{0}\right)\right)$ versus footpoint field strength $\left(\log _{10}\left(B_{0}\right)\right)$; and (b) proton flux density at Earth $\left(\log _{10}\left(n_{\mathrm{E}} v_{\mathrm{E}}\right)\right)$ versus proton flux density at the coronal base $\left(\log _{10}\left(n_{0} v_{0}\right)\right)$. Here, mass conservation and source surface extrapolations of the observed photospheric field have been used to derive $n_{0} v_{0}$ from solar wind measurements at $A C E$ during 1998-2009 (see Wang, 2010). The mass flux density at the coronal base increases almost linearly with the footpoint field strength, but the mass flux density at Earth undergoes relatively little variation. This is because the increase in the heating rate and mass flux density with $B_{0}$ is offset by a corresponding increase in the expansion factor.

coronal holes of the same polarity. The open flux tubes rooted alongside pseudostreamers initially diverge rapidly with height, but then re-converge as they approach the source surface; the local expansion factor $f(r)=\left(R_{\odot} / r\right)^{2}\left(B_{0} / B\right)$ thus reaches its maximum value $f_{\max }>f_{\mathrm{ss}}$ at some heliocentric distance $r<R_{\mathrm{ss}}$. Comparisons with near-Earth solar wind measurements indicate that the wind speeds associated with pseudo-streamer crossings are lower than predicted using the inverse correlation between $v$ and $f_{\text {ss }}$ (see Riley and Luhmann, 2012; Wang et al, 2012). This suggests that the empirical model should be amended by replacing the parameter $f_{\mathrm{ss}}$ by $f_{\max }$, defined as the maximum value of $f(r)$ along an open flux tube; $f_{\max }$ is identical to $f_{\mathrm{ss}}$ for monotonically expanding flux tubes, but exceeds $f_{\mathrm{ss}}$ for flux tubes near pseudo-streamer boundaries.

A recent study by Pinto et al (2016) has tested whether the flux-tube expansion is the controlling factor of the wind speed at all phases of the cycle and at all latitudes (close and faraway from streamer boundaries) using a very large sample of wind-carrying open magnetic flux-tubes created by numerical MHD simulations of the corona and wind coupled to a dynamo model. This study confirms that the asymptotic speed of the solar wind depends very strongly on the geometry of the open magnetic flux-tubes through which it flows, but specially for the SSW, the speed is also strongly dependent on field-line inclination and magnetic field amplitude at the foot-points. 
4.2 Interchange reconnection vs. expansion factor models

The interchange reconnection model (Fisk et al, 1998; Fisk and Schwadron, 2001; Fisk, 2003; Zurbuchen, 2006; Fisk and Zhao, 2009) discussed above as scenario SSW2 for the slow solar wind formations, in which the small-scale dynamics of the photospheric magnetic field, as in the so-called magnetic carpet (e.g., Schrijver et al, 1997), play the central role. In many respects, the interchange reconnection model can be considered to be the diametric opposite of the expansion factor scenario. Unlike the expansion factor model, which assumes a quasi-steady field, the magnetic field is inherently dynamic in the interchange model, and this dynamics are the essential feature of the model. Also, unlike the expansion factor model in which the slow wind originates from the open-field coronal holes, the slow wind in the interchange reconnection model is postulated to originate from throughout the closed-field region via continuous interchange reconnection between open and closed flux. Note that in the interchange reconnection model the closed field regions are not truly closed in that open flux is assumed to diffuse throughout these regions via reconnection.

The interchange reconnection model has obvious advantages in accounting for slow wind observations: it naturally produces a highly variable wind with closed-field plasma composition, located around the HCS but with large extent. The primary challenge for the model is to verify that interchange reconnection induced by photospheric dynamics does, indeed, enable open flux to diffuse deep into closed-field regions. Simulations have found that the open-closed boundary remains smooth and well defined, even during interchange reconnection (Edmondson et al, 2009, 2010; Linker et al, 2011). Furthermore Antiochos et al (2007) have proposed semianalytic theorems that severely constrain the possible topologies of the Sun's open flux. To this hypothesis, the SSW originates from an interface region between narrow coronal holes and closed field lines (S-web model, see Section 4.3; Antiochos et al, 2011) in according to the boundary layer scenario described by Riley and Luhmann (2012). On the other hand, all the calculations, to date, have lacked the topological complexity of the observed photospheric flux; therefore, it remains to be seen whether interchange reconnection in a sufficiently complex magnetic topology can produce effective mixing of open and closed flux.

Rappazzo et al (2012) showed that reconnection can occur continuously in unipolar magnetic field regions with no neutral points: photospheric motions induce an MHD turbulent cascade in the coronal field that creates the necessary small scales, where a sheared magnetic field component orthogonal to the strong axial field is created locally and can reconnect. They propose that a similar mechanism operates near and around boundaries between open and closed regions inducing a continual stochastic rearrangement of connectivity, originating the SSW.

\subsection{S-web model}

S-Web model of the SSW contains elements of both the expansion factor and interchange reconnection scenarios. Given its associations with the HCS, its variability, 


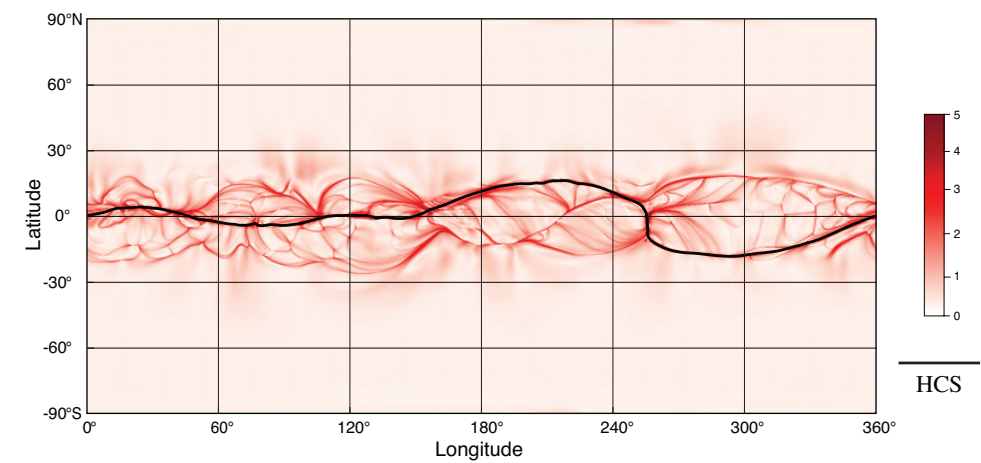

Fig. 19 Distribution of Q-factor on a heliospheric surface at $10 \mathrm{R}_{\odot}$ for a CR centered about August 1, 2008, during the middle of solar minimum. The HCS appears as the thick black line and the contours of high $\mathrm{Q}$ as red $\left(\log _{10}\right.$ scale). The HCS has infinite Q. From Antiochos et al (2011).

and especially its composition, an appealing scenario is that the slow wind is due to the release of closed field plasma onto open field lines at the open-closed boundary (e.g., Suess et al, 1996; Endeve et al, 2004; Rappazzo et al, 2005, 2012). This is the underlying hypothesis of the interchange reconnection model (see the previous section), but in the S-Web, the magnetic topology is assumed to be smooth as in the expansion factor model, with well-defined open and closed field regions. The release of closed field plasma is postulated to occur only in a narrow layer about the open-closed boundary. The model, therefore, is dynamic rather than quasi-static, but the dynamics are confined to this narrow layer.

According to the S-Web model a dynamic interface layer about the open-closed boundary is the source of the slow wind. Note also that the other two models depend heavily on the properties of the open-closed boundary; consequently, this boundary is critical for determining heliospheric structure and dynamics. It might seem that the open-closed boundary would have only limited impact on the heliosphere, however, because it is simply the boundary of coronal holes. The field lines at this boundary define the edges of the streamer belt and extend out into the heliosphere as the heliospheric current sheet (HCS). Therefore, other than some narrow region about the HCS, one might expect that any dynamical evolution of the open-closed boundary would have minimal effect in the heliosphere, especially during solar minimum when the streamer belt and the HCS are confined near the equatorial plane and have relatively simple geometry.

Figure 19 shows that this picture is completely incorrect. Plotted in the Figure are contours of the so-called squashing factor Q (Titov et al, 2002) for the fieldline mapping from a heliospheric surface at $10 \mathrm{R}_{\odot}$ down to the photosphere. The magnetic field is from a quasi-steady model calculated by a MHD code, with SOHO/MDI measurements of the photospheric field during a CR centered about August 1, 2008 as input (Mikić et al, 2007). Note that Fig. 19 is model-independent; identical results would be obtained if one were to use a high-resolution source surface model instead of MHD. 
$\mathrm{Q}$ is a measure of the gradients in the field line mapping. At a high $\mathrm{Q}$ region, a small circular area, for example, on the $10 \mathrm{R}_{\odot}$ surface is mapped by the field lines into a highly distorted ellipse on the photosphere. At separatrices where the mapping becomes singular, as in the HCS, Q is infinite. Note, however, that the HCS clearly is not the only high Q curve in Fig. 19; instead we see a dense web of high-Q curves indicating the location of separatrices or quasi-separatrices in the topology of the heliospheric field. This is the S-Web (Antiochos et al, 2011).

The key point of the S-web in Fig. 19 is that every high-Q curve (including the HCS) maps down to the open-closed boundary or passes extremely close to such a boundary. Therefore, any dynamics associated with the open-closed boundary field line opening and closing and interchange reconnection (Crooker et al, 2002) will affect a broad swath of the inner heliosphere, not just a narrow region about the HCS. This result seems paradoxical, because we know that the open-closed boundaries of coronal holes must map directly to the HCS; however, Fig. 19 shows that the HCS can lie far from some of the high-Q curves. On the other hand, the conclusion that the high- $\mathrm{Q}$ arcs are due to open-closed boundaries is inescapable, because this is the only way to obtain topological structure in the open field mapping. The magnetic topology is essentially smooth in the heliosphere where all flux is open, so any topological singularities in the mapping can only be due to the effect of closed flux. For example, the HCS has singular Q only because the helmet at its base surrounds closed field.

Recent works (Antiochos et al, 2007, 2011, 2012) resolve the paradox of the high-Q arcs in Fig. 19, where the field is both near the open-closed boundary and yet far from the HCS. The critical new insight is that seemingly separate coronal holes must be topologically connected by magnetic flux (Antiochos et al, 2007). We show below that such flux will connect to the coronal hole boundary in the corona, but diverge far from the HCS in the heliosphere.

\subsubsection{The origin of $S$-web arcs}

In some phases of the solar cycle, a major form of open-closed topology in the corona is a pseudo-streamer (Wang et al, 2007b), originally identified by Hundhausen (1972) and referred to as a plasma sheet or unipolar streamer (e.g., Riley and Luhmann, 2012). The topology of the open-closed boundary of a pseudostreamer is vital for understanding the inner heliosphere, because it produces almost all of the high-Q arcs of the S-Web. There is generally only one streamer belt on the Sun and, therefore, only one HCS in the heliosphere, but as Fig. 19 shows, the high-Q arcs of pseudo-streamers can fill a large fraction of the inner heliosphere. The pseudo-streamer Q signature appears as a finite-length curve, perhaps of finite width, that starts and ends on the HCS. These arcs are prevalent in Fig. 19 and in Q-maps of other CRs (e.g., Titov et al, 2012).

Because all high-Q arcs of Fig. 19 begin and end at the HCS, their origin must involve the coronal hole. In fact, the most insightful approach to understanding these arcs is to consider the interaction of the coronal hole boundary with closed field regions due to nearby parasitic polarity. One example of such an interaction is shown in Fig. 20. The field sources are a global dipole that gives rise to the 


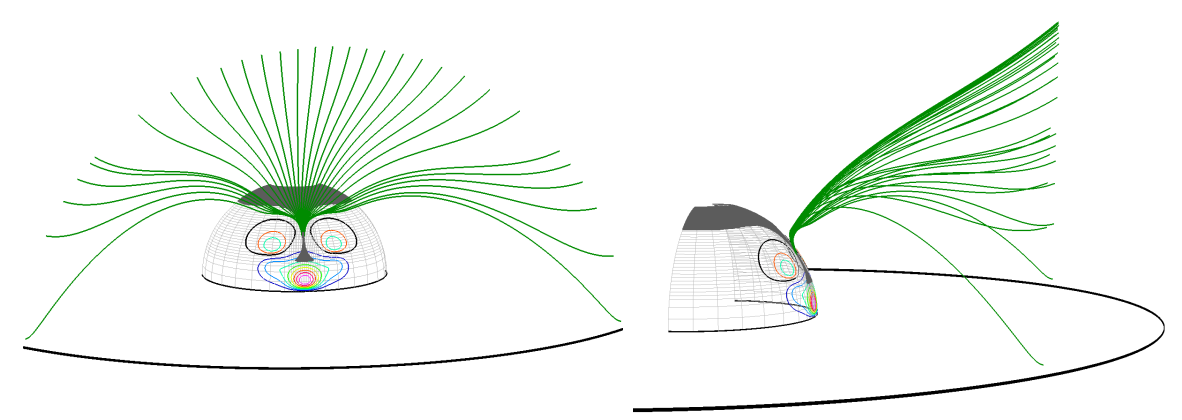

Fig. 20 The magnetic field of a PFSS model in which a low-latitude coronal hole extension is connected to the polar hole by a finite width corridor. The left panel shows the front view and the right panel the side view. Dark gray shaded regions indicate open flux. Contours at the inner surface indicate radial field magnitude at the photosphere. Black lines are polarity inversion lines at the photosphere and at the source surface (the start of the HCS); green field lines are traced from a latitudinal line segment spanning the narrowest part of the corridor. Their footpoints on the source surface trace out one of the high-Q arcs evident in Fig. 19. From Antiochos et al (2011).

polar coronal holes and an equatorial near-surface dipole that creates a frequently observed "elephant trunk" extension of the polar coronal hole (Timothy et al, 1975; Zirker, 1977). The addition of two near-surface dipoles at high latitudes produces two parasitic polarity regions (indicated by the two high-latitude polarity inversion lines, PILs) on either side of the elephant trunk. Note that these parasitic polarities always produce closed flux surrounding their PILs; consequently moving the two parasitic polarities closer together causes their fan surfaces to "squeeze" the open field region into a narrow corridor connecting the polar hole with its equatorial extension.

These connections between a polar coronal hole and its equatorial extensions are the topological structures that give rise to pseudo-streamers and to the S-Web arcs of Fig. 19 (Antiochos et al, 2011; Titov et al, 2011). The high-Q arcs are not due to some fractal complexity of the coronal-hole boundary itself. The coronal hole boundary, no matter how complex, maps only to the HCS, and hence cannot account for the high-Q arcs far from the HCS. However, by simply mapping the open-field corridor flux of Fig. 20 onto the $10 \mathrm{R} \odot$ surface, we find that it forms a high-Q arc exactly like those of Fig. 19. This arc would appear as a pseudostreamer in a coronagraph image.

An important point is that the width of the corridor, and consequently the width of the high-Q arc in the heliosphere, can be infinitesimal, i.e., a separatrix singularity. For example, if the high latitude dipoles of Fig. 20 moved so close together that their PILs at the photosphere merged, then the resulting topology would consist of a polar coronal hole with an equatorial extension that is linked to the polar one via separator curves connecting multiple null points in the corona (Titov et al, 2011). It may seem that such multiple null points with separator lines nor the narrow open field corridors of Fig. 20 would form often on the Sun, but in fact, they are common, especially during solar maximum (e.g., Crooker et al, 2012). In 
the so-called "rush to the poles" of trailing polarity flux during the solar cycle, long tongues of opposite polarity often cut into coronal hole regions, leading to the pseudo-streamer structures illustrated by Fig. 20 .

In summary, the S-web model can account for the large angular extent of the slow wind about the HCS, once the complexity of the coronal magnetic topology is fully included in the model. Furthermore, it provides a straightforward explanation for all the temporal and compositional differences between the slow and fast winds; the slow is due to the sporadic release of closed field plasma onto open field lines, whereas the fast is the quasi-steady wind of Parker on long-lived open flux. It should be emphasized, however, that the studies to date have focused only on the quasi-static topological properties of the S-web, such as the results shown in Figs. 19 and 20. A great deal more work needs to be done in order to determine whether fully dynamic models of the S-web do reproduce all the observed properties of the SSW.

\subsection{Magnetic reconnection at the streamer cusp}

Magnetic reconnection (Priest and Forbes, 2000) is a key process in many scenarios that have been proposed to explain coronal processes. Focusing on SSW origins, the process at the cusp of helmet streamers (Lapenta and Knoll, 2005a), the formation of plasma blobs in the SSW (Einaudi et al, 2001) and the interaction between open and closed field lines (Antiochos et al, 2011) all involve reconnection. The question is: what type of reconnection is likely to be actually happening there? The recent research on reconnection has identified different regimes depending on the parameters of the physics problem. Figure 21 summarizes the different regimes in a diagram of the Lundquist number $S_{L}=\mu_{0} L V_{A} / \eta$ versus the thickness of the reconnecting layer normalized to the ion inertial length $\delta_{i}=L / d_{i}$ (Ji and Daughton, 2011; Baalrud et al, 2011). At the largest scale, the single fluid MHD description is valid. The Sweet (1958)- Parker (1957) (SP) and the Petschek (1964) steady regimes have been identified early in the history of the research on reconnection. Somewhat later, a third unsteady regime has been suggested (Bulanov et al, 1979) as a consequence of the destabilization of a SP layer by a modified tearing instability. The reconnecting layer being itself a thin current can be subject to tearing, but the presence of a flow of plasma around the current alters the stability and growth rate of the pure tearing mode (Loureiro et al, 2007). This regime (lower right of the diagram) is accessible only to relatively non-collisional, high $S_{L}$ plasmas. The reconnection process becomes fast and insensitive to resistivity but also unsteady and characterized by secondary plasmoids (Lapenta, 2008; Cassak et al, 2009; Samtaney et al, 2009; Huang and Bhattacharjee, 2010). Only recently, this regime has become accessible to direct numerical simulation (Lapenta, 2008). Its existence required $S_{L}$ values that became possible only in the days of massively parallel computers. The transition between the SP and the plasmoid regime has been predicted (Loureiro et al, 2007) and shown in practice (Skender and Lapenta, 2010; Bhattacharjee et al, 2009; Uzdensky et al, 2010) to happen at a critical value of the Lundquist number $S_{C}=10^{4}$. 
At the smallest scales, when the ion inertial length $d_{i}$ is larger than the current sheet thickness predicted by Sweet-Parker model $\delta_{S P}=L S_{L}^{-1 / 2}$ (upper left of the diagram), the kinetic regime of fast reconnection develops (Birn and et al., 2001). This regime is characterized by a separation of scales between ions and electrons. In an outer ion diffusion region, the ions become decoupled from the field lines and in an inner electron diffusion region, the electrons become also decoupled. While single fluid MHD completely misses this regime, some aspects can be captured by two-fluid and hybrid (fluid electrons and kinetic ions) models. Only full kinetic models can capture this regime fully. In the kinetic regime, the presence of the plasmoid instability is still possible. The electron diffusion region forms elongated jets (Karimabadi et al, 2007) and tearing is possible leading to the formation of plasmoids and the transition to an unsteady rate of reconnection (Wan and Lapenta, 2008a). Additionally, a current layer can tear in multiple points with the formation of several interacting islands (Markidis et al, 2012, 2013; Eriksson et al, 2014). The subsequent evolution is characterized by merging of the primary structures formed by tearing (Karimabadi et al, 2005; Wan and Lapenta, 2008b).

At intermediate scales when $\delta_{P L}<d_{i}<\delta_{S P}$ Ji and Daughton (2011), where the resistive skin depth for plasmoid is $\delta_{P L}=\delta_{S P}\left(V_{A} / L / \gamma\right)^{1 / 2}$, and $\gamma$ is the plasmoid growth rate, an intermediate regime is present where secondary plasmoids are formed even though the ion-electron separation of scales is present. In all regimes, for sufficiently low collisionality, unsteady chaotic reconnection is promoted by plasmoid formation due to the tearing on the thinner layers formed in earlier phases of the reconnection process.

Determining the location of the regions of origin of the SSW on the diagram in Fig. 21 is not unambiguous. In the regions of the corona where the SSW forms, classical collisional resistivity is very small. However, it could be argued that kinetic micro instabilities provide an effective collisionality that produces anomalous resistivity and lowers the Lundquist number. Based on pure collisionality, in the diagram in Fig. 21, the position of the corona is well above the threshold for plasmoid instability, making it likely that the true nature of the reconnection processes ranges from plasmoid to Hall plasmoid to even fully kinetic reconnection. A similar ambiguity is present for determining the position on the horizontal axis. When considered in their visual scales (Einaudi et al, 1999), the regions of formation of the SSW are larger than the ion skin depth, making the plasmoid regime at first sight the most likely scenario. However, there is a whole range of features hidden inside the large scales that can be determined for example from a LASCO coronagraph image (see Section 2.2). The presence of turbulence and current filamentation might hide the presence of thinner current layers embedded within the macroscopic structure (Bemporad et al, 2006; Bemporad, 2008). This possibility cannot be fully determined from remote sensing and a final answer will come only from in situ detection via probes. The presence of sub scale features is of great importance. The research on turbulent reconnection (e.g., Lazarian and Vishniac, 1999; Matthaeus and Lamkin, 1986) has shown that even very thick reconnecting strata can evolve very quickly compared with the SP scenario (Eyink et al, 2011), favoring the mechanisms that lead to SSW formation (Lapenta and Restante, 2008). Even in absence of pre-existing turbulence in the ambient plasma, the plasmoid regimes of reconnection are insensitive to resistivity (Bhattacharjee et al, 2009) and derive their much faster rates (Lapenta, 2008), when compared to 
the SP reconnection process, to the presence of smaller features within the reconnecting layers (Lapenta and Lazarian, 2012): the ion and electron scales of kinetic reconnection or the plasmoid scales of the plasmoid regimes.

Whether turbulence is assumed to be present already or not, the end state is nevertheless one of chaotic reconnection with multiple reconnection sites, on a range of different, possibly fractal or self-similar scales with large layers breaking off in smaller layers further breaking off into yet smaller layers, until the kinetic limit of reconnection is reached when the current sheet thickness on the smallest scales reaches the local ion inertial length scale (Daughton et al, 2009). In the specific case of the models for the SSW formed at the cusp of helmet streamers (Einaudi et al, $1999,2001)$, the process of reconnection is intertwined with momentum exchange across field lines. The state includes both magnetic shear and velocity shear. At the cusp, the plasma frozen into the closed field lines of the helmet streamer is stationary while that frozen into open field lines emerging from coronal holes is moving at the speed of the fast wind. A strong velocity shear is then present along with the magnetic shear typical of the helmet topology. This state is subject to both tearing and Kelvin-Helmholtz instability (Ofman et al, 1991; Einaudi et al, 1999; Ofman et al, 1993; Knoll and Chacón, 2002). The presence of the flow can itself promote the rate of reconnection because the flow acquires a converging nature and drives the process of reconnection pushing the field lines together. The KHI and the cusp topology divert the flow from its direction parallel to the field lines driving reconnection (Lapenta and Knoll, 2005b). The transition to the chaotic plasmoid reconnection regimes speeds up the rate of reconnection, promoting a faster exchange of momentum from the surrounding fast solar wind to the plasma released in the reconnection above the helmet streamers (Lapenta and Restante, 2008; Bettarini and Lapenta, 2010).

\subsection{Discussion}

Figure 17 describes several scenarios for the SSW formation. The implementation of these scenarios in quantitative numerical models should give predictions on plasma properties such as densities, temperatures, non-thermal heating of the plasma constituents, magnetic field fluctuations and ultimately the velocity distributions of the plasma components. This implies a challenge to the theorists and space weather modelers in the field.

From the observational point of view, at the Sun, one would expect that expansion factor scenario (SSW1 relative to Fig. 17) should relate to properties of the coronal hole close to but inside the coronal hole boundary and carry kinetic signatures of nonthermal heating, if any. If interchange reconnection is important (SSW2 relative to Fig. 17) then the in-situ measurements in the heliosphere would relate to the closed-loop properties just outside of the coronal hole boundary. At $1 \mathrm{AU}$, SSW2 plasma would be found around the narrow plasma sheet between two solar wind streams, while the SSW1 plasma can have a certain spatial extent away from the plasma sheet (Wang et al, 2000). Moreover, both SSW1 and SSW2 scenarios postulate that the source is $\sim 30,000 \mathrm{~km}$ wide layer about open-closed boundary, but expansion states layer is in open field only, while interchange reconnection 


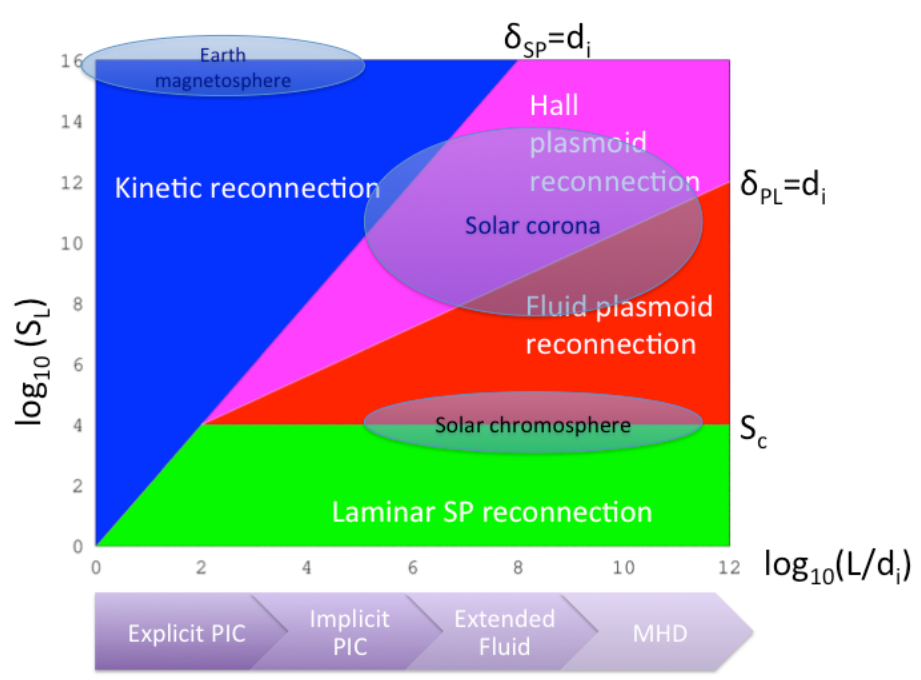

Fig. 21 Diagram of different reconnection scenarios versus normalized reconnecting current layer thickness $\delta_{i}=L / d_{i}$, and Lundquist number $S_{L}$. Other designations in the figure are the following: $\delta_{S P}$ - current sheet thickness predicted by Sweet-Parker model; $\delta_{P L}$ - resistive skin depth for plasmoid; $S_{C}$ - critical value of the Lundquist number for the onset of plasmoid instability. Also shown are typical parameter ranges for a few plasmas of interest in space research: the Earth magnetotail, the solar chromosphere and the solar corona. The different simulation methods best suited to study each scale are also set in the diagram according to their domain of applicability.

model claims dynamic opening and closing. SSW3 hypothesis (relative to Fig. 17) is linked to events describing a sporadic and highly fluctuating emission of plasma. Questions remain whether the SSW2 and SSW3 scenario can represent the bulk SSW, or whether they only partially account for the solar wind outflow that is transient in nature.

The above scenarios do not need to be mutually exclusive and may very well coexist. Observations show signatures consistent with all of these scenarios, and so far none are excluded. The SSW1 scenario is a natural consequence of the global coronal magnetic field model. Its empirical prediction of the solar wind speed at 1 AU provides a certain level of support (Wang and Sheeley, 1990; Arge and Pizzo, 2000). Results from SOHO/UVCS observations of streamers and coronal hole boundaries (see Section 2.1.2) also are compatible with this picture. The SSW2 scenario provides several predictions that are consistent with observations. Crooker et al $(2004,2010)$ found evidence of interchange reconnection in the suprathermal electron properties at the heliospheric plasma sheet/current sheet and the sector boundary, as compared to the interplanetary magnetic field data. Jets and transient brightenings at the coronal hole boundary implies occurrences of magnetic reconnection caused by the interaction of the dynamic field transport as well as emerging close loops with the open field in the coronal hole (Madjarska et al, 2004, 2012; Madjarska and Wiegelmann, 2009; Subramanian et al, 2010). The observational evidence from a large number of Hinode data analyses indicates 
that the continuous expansion of active regions and its interaction with nearby open field can lead to the process of interchange reconnection, and therefore such outflows represent a possible contribution to the SSW2 (see Section 2.1.1). Blobs flowing along streamer stalks (e.g., Sheeley et al, 1997) and depleted elemental abundances near the heliospheric current sheet (Suess et al, 2009; Weberg et al, 2012) support the idea of streamer material released by field pinching at the top of the streamer cusp/current sheet (SSW3 scenario).

\section{Which are the main physical mechanisms for the SSW acceleration?}

There are many differences between slow and fast wind in heliosphere such as, for example, velocity, proton flux density, temperatures, heavy ion relative abundances, $\alpha$ 's content and variability degree that suggest different heating and acceleration mechanisms for the slow and fast solar wind. The velocity distribution of the protons as measured in situ by Helios spacecraft at $\sim 0.3 \mathrm{AU}$ and farther from the Sun, shows that the SSW plasma is generally more Maxwellian, and does not exhibit significant temperature anisotropy and beams, contrary to the fast solar wind proton velocity distribution (e.g., Marsch, 1991). But, there are also some important similarities such as the momentum flux and the total energy flux that provide likely clues to the physical processes for the formation and acceleration of the solar wind (Le Chat et al, 2012). The possibility of wave-driven wind has been applied extensively mostly to fast solar wind models (see the review, Ofman, 2010), and in principle, it can be applied also to the SSW.

At present, several physical mechanisms that can produce the solar wind are considered as the main candidates: MHD and kinetic waves, shocks, turbulence, energy release by reconnection, and direct acceleration by the Lorentz force of plasmacarrying ejected magnetic flux (e.g., plasmoids, as deduced from SOHO/LASCO white light observations by Sheeley et al (1997), and recently by STEREO/SECCI by Viall and Vourlidas (2015)). MHD waves can drive the slow wind as well accounting for the variability, while kinetic (resonant) waves can provide the differential heating of ions often observed in the solar wind plasma. In particular, MHD waves can heat and accelerate the SSW in the open field regions surrounding a helmet streamer. The wave driven wind model is closely connected to Alfvénic turbulence driven wind in the low frequency domain (e.g., Cranmer and van Ballegooijen, 2005). It is evident that most of these mechanisms could be related, for example, the broadband Alfvén waves and weak Alfvénic turbulence are similar, the small-scale kinetic Alfvén and ion-cyclotron waves can results from turbulent cascade starting from large (MHD) scales, reconnection can produce shocks, waves, and jets, while the later can produce turbulence and waves leading to the plasma heating.

The relation between the proton temperature anisotropy and the parallel temperature of protons as observed by ACE and WIND spacecraft show that the solar wind plasma parameters are in a range bound by the threshold of various kinetic instabilities, providing important clues on the possible kinetic processes involved in the formation of the solar wind plasma (Bale et al, 2009; Maruca et al, 2011, 2012). 
The magnetic fluctuations spectra in the solar wind as observed in situ by the various spacecraft often exhibit power law scaling with turbulent spectrum in the inertia range that agrees with Kolmogorov turbulence scaling of $-5 / 3$, and steeper slope in the high frequency (above the proton gyroresonat frequency) dissipation range (see the review Bruno and Carbone, 2005), suggesting that turbulence plays a role in transferring magnetic fluctuation energy from large scales to small scales and leading to the heating of the solar wind plasma in the (ion) kinetic scales. It is often suggested in the literature that the high perpendicular temperature and the temperature anisotropy of $\mathrm{O}^{5+}$ ions deduced from UVCS/SOHO spectroscopic observations in coronal holes provides evidence of ion-cyclotron wave heating in the extended solar corona (e.g., Cranmer et al, 1999b). Frazin et al (2003) and Susino et al (2008) showed that temperature anisotropy of $\mathrm{O}^{5+}$ is also present in coronal streamers, albeit at a lower degree than in coronal holes. In-situ observations by WIND spacecraft show a link between proton-He ${ }^{++}$differential streaming and the degree of heating of the solar wind ions suggesting ion-cyclotron wave heating (Kasper et al, 2013). Thus, it is plausible that ion-cyclotron waves can heat the ions in the SSW plasma, but to lower kinetic temperatures.

It has been suggested decades ago that low-frequency MHD (Alfvén) waves can provide significant momentum to accelerate the fast solar wind in open field regions of coronal holes (see the review by Ofman, 2010). However, the SSW can be accelerated to the observed velocities just by the thermal pressure gradient of the hot solar wind plasma, as was shown in the original work by Parker (1958). Thus, additional momentum input by wave pressure is not required to produce the SSW. However, whether MHD waves are involved in the heating and the acceleration of the SSW is still an open question. Measurement of possible observational signatures of the various mechanisms in the acceleration region of the solar wind close to the Sun such as planned by the future missions Solar Probe Plus (SPP) and Solar Orbiter (SO) may provide the necessary evidence to pinpoint the heating and the acceleration processes that produce the solar wind.

\section{Conclusions}

In this review, we have presented insights on the main SSW sources and their formation mechanisms based on leading observations and modeling approaches arisen during the discussions at the International Space Science Institute (ISSI) by the Team entitled "Slow solar wind sources and acceleration mechanisms in the corona" held in Bern (Switzerland) in March 2014 and 2015. From the remote sensing observational results in the corona, it seems that there are different contributions to the SSW outflow due to the large and small scale structures in corona and with both sporadic and continuous fluxes. In-situ measurements are available from $0.3 \mathrm{AU}$ and beyond, and provide the bulk quantities data such as outflow velocity, density, and temperature of the solar wind, together with the magnetic fluctuations, ionic composition, and in some cases velocity distribution functions. We propose to classify the SSW not only on the basis of the outflow velocity and coronal sources but considering also the elemental composition and the heavy ion charge state which are fixed in the inner corona and therefore intimately related to 
the properties in the solar wind source regions. The main question is to quantify the mass and energy budgets of these different SSW components.

There are two main general classes of solar wind acceleration and heating mechanisms supported by observations: reconnection and waves/turbulence, where the waves could range from MHD to kinetic dissipation scales. It is convenient to classify the SSW formation and origin theories using three general hypotheses that combine the source regions, magnetic topology, and relate to the above acceleration and heating mechanisms: (a) SSW produced in regions of open magnetic field with large expansion factor that leads to smaller solar wind speed than in open field regions with smaller expansions factor, in this case the acceleration and heating by turbulence and waves is favored. This mechanism is similar to the fast solar wind formation mechanism that is often associated with coronal holes, but the open field has higher expansion factor, and the source of the plasma is in the streamer/coronal hole boundary regions. The reconnection driven SSW includes the (b) interchange reconnection, which relates to the interaction of emerging closed field region with open field. In this case the reconnection provides the heating and the acceleration of the solar wind emerging from closed field plasma. The S-web model is a distinctive scenario where reconnection occurs in closed field region along separatrices in the lower corona. This mechanism can also take place in streamers and pseudo streamers through reconnection at cusp. Plasmoid eruption is also related to this scenario since reconnection and the resulting Lorentz force accelerate the wind. The combination of the heating acceleration mechanisms, the source regions, and magnetic topologies provides the SSW formation scenarios (SSW1, SSW2 and SSW2, as defined above in Section 4). These hypotheses may explain many but not all the observational aspects of the SSW as discussed in Section 4.5.

Given these scenarios, the next sensible step would be to investigate how the solar properties at the solar wind source regions dictate the properties of the solar wind in order to find out likely mechanisms and processes responsible for the solar wind formation and further constrain solar wind models. To do so it would be ideal if, for a given solar wind parcel, the location of its footpoint at its source coronal hole could be identified. Unfortunately, specific footpoint locations are still very difficult to identify with acceptable level of confidence. This measurement gap may be filled with appropriately detailed, physics based numerical models, while the quests for observational evidences of slow wind formation will continue. More investigations are needed to look for causal relationships between solar wind properties and that in its source regions.

The SSW origin is an open issue in the post SOHO era and forms a major objective for planned future missions such as SO and SPP. In particular, the SPP mission will provide solar wind measurements close to the sun up to $\sim 9.5 \mathrm{R}_{s}$ in the unexplored acceleration region, and may confirm or refute the SSW formations scenarios discussed in the present review. The proposed hypotheses need to make predictions on the plasma and magnetic properties that SO and SPP will be able to measure. Since these measurements will include kinetic data in addition to bulk fluid properties, MHD modeling alone is not sufficient to address the physics of the anticipated measurement of the SSW, and multi-fluid and kinetic approaches must supplement the modeling efforts. SO observations, performed during quasi- 
corotation and out-of-ecliptic, will provide major contribution to the study of the SSW sources and association with large-scale magnetic structures. They will allow analyzing the evolution of the streamer belt and global corona (large-scale structures) separating the rotational effect and intrinsic evolution. Quasi-corotation allows observing the streamer belt on time scales $>2-3$ days, which is the passage time of structures at the limb and to study the role of the coronal magnetic field topology in guiding the wind and regulating speed and physical parameters through the solar cycle. The quadratures between SO and SPP can provide clues for identifying SSW coronal sources with composition and dynamics measurements and thus improve our understanding of the acceleration and heating mechanisms. There are still many open questions on SSW sources and acceleration but we hope that this review can be useful for future observation planning with SO and SPP.

Acknowledgements This review has arisen from the discussions at the International Space Science Institute (ISSI) by the Team entitled "Slow solar wind sources and acceleration mechanisms in the corona" held in Bern in March 2014-2015, and we acknowledge ISSI support for the meetings. We would like to thank the referees for many helpful comments that helped to improve this review. LA acknowledges Giancarlo Noci for his input and comments during the revision of this paper. The research of LA has been funded through the contract I/023/09/0 between the National Institute for Astrophysics (INAF) and the Italian Space Agency (ASI). LO would like to acknowledge support by NSF grant ATM AGS-1059838 and NASA Cooperative Agreement grant NNG11PL10A to CUA. YKK, LS and YMW would like to acknowledge support by the Chief of Naval Research and NASA grant NNH10AO82I.

\section{References}

Abbo L, Antonucci E, Dodero MA, Mikić Z, Riley P (2006) Slow Coronal Wind Composition. In: SOHO-17. 10 Years of SOHO and Beyond, ESA Special Publication, vol 617, p 17

Abbo L, Antonucci E, Mikić Z, Linker JA, Riley P, Lionello R (2010a) Characterization of the slow wind in the outer corona. Advances in Space Research 46:1400-1408, DOI 10.1016/j.asr.2010.08.008, 1008.4452

Abbo L, Ofman L, Giordano S (2010b) Streamers study at solar minimum: combination of UV observations and numerical modeling. Twelfth International Solar Wind Conference 1216:387-390, DOI 10.1063/1.3395883

Abbo L, Lionello R, Riley P, Wang YM (2015) Coronal Pseudo-Streamer and Bipolar Streamer Observed by SOHO/UVCS in March 2008. ArXiv e-prints 1505.05649

Akinari N (2007) Morphological Study of Quiescent Streamers during Solar Minimum by Ultraviolet Emission Lines. Astrophys. J.668:1196-1209, DOI $10.1086 / 521386$

Antiochos SK, DeVore CR, Karpen JT, Mikić Z (2007) Structure and Dynamics of the Sun's Open Magnetic Field. Astrophys. J.671:936-946, DOI 10.1086/522489, 0705.4430 
Antiochos SK, Mikić Z, Titov VS, Lionello R, Linker JA (2011) A Model for the Sources of the Slow Solar Wind. Astrophys. J.731:112, DOI 10.1088/0004$637 \mathrm{X} / 731 / 2 / 112,1102.3704$

Antiochos SK, Linker JA, Lionello R, Mikić Z, Titov V, Zurbuchen TH (2012) The Structure and Dynamics of the Corona-Heliosphere Connection. Sp. Sci. Rev.172:169-185, DOI 10.1007/s11214-011-9795-7

Antonucci E (2006) Wind in the Solar Corona: Dynamics and Composition. Sp. Sci. Rev.124:35-50, DOI 10.1007/s11214-006-9098-6

Antonucci E, Abbo L, Dodero MA (2005) Slow wind and magnetic topology in the solar minimum corona in 1996-1997. Astron. Astrophys.435:699-711, DOI 10.1051/0004-6361:20047126

Antonucci E, Abbo L, Telloni D (2006) Oxygen Abundance and Energy Deposition in the Slow Coronal Wind. Astrophys. J.643:1239-1244, DOI 10.1086/503186

Antonucci E, Abbo L, Telloni D (2012) UVCS Observations of Temperature and Velocity Profiles in Coronal Holes. Sp. Sci. Rev.172:5-22, DOI 10.1007/s11214010-9739-7

Arge CN, Pizzo VJ (2000) Improvement in the prediction of solar wind conditions using near-real time solar magnetic field updates. J. Geophys. Res.105:10,46510,480, DOI 10.1029/1999JA000262

Arge CN, Henney CJ, Koller J, Compeau CR, Young S, MacKenzie D, Fay A, Harvey JW (2010) Air Force data assimilative photospheric flux transport (ADAPT) Model. In: Maksimovic, M., Issautier, K., Meyer-Vernet, N., Moncuquet, M., Pantellini, F. (eds.). Twelfth International Solar Wind Conference, AIP Conf Proc 1216:343-346, DOI 10.1063/1.3395870

Athay RG (1986) Radiation loss rates in Lyman-alpha for solar conditions. Astrophys J 308:975-981, DOI 10.1086/164565

Baalrud SD, Bhattacharjee A, Huang YM, Germaschewski K (2011) Hall magnetohydrodynamic reconnection in the plasmoid unstable regime. Physics of Plasmas 18(9):092,108, DOI 10.1063/1.3633473, 1108.3129

Baker D, van Driel-Gesztelyi L, Mandrini CH, Démoulin P, Murray MJ (2009) Magnetic Reconnection along Quasi-separatrix Layers as a Driver of Ubiquitous Active Region Outflows. Astrophys. J.705:926-935, DOI 10.1088/0004637X/705/1/926, 0909.4738

Baker D, Brooks DH, Démoulin P, Yardley SL, van Driel-Gesztelyi L, Long DM, Green LM (2015) FIP Bias Evolution in a Decaying Active Region. Astrophys. J.802:104, DOI 10.1088/0004-637X/802/2/104, 1501.07397

Bale SD, Kasper JC, Howes GG, Quataert E, Salem C, Sundkvist D (2009) Magnetic Fluctuation Power Near Proton Temperature Anisotropy Instability Thresholds in the Solar Wind. Physical Review Letters 103(21):211101, DOI 10.1103/PhysRevLett.103.211101, 0908.1274 
Bame SJ, McComas DJ, Barraclough BL, Phillips JL, Sofaly KJ, Chavez JC, Goldstein BE, Sakurai RK (1992) The ULYSSES solar wind plasma experiment. Astron. Astrophys. Supp.92:237-265

Barakat AR, Schunk RW (1982) Transport equations for multicomponent anisotropic space plasmas - A review. Plasma Physics 24:389-418, DOI 10.1088/0032-1028/24/4/004

Beckers JM, Chipman E (1974) The Profile and Polarization of the Coronal L $\alpha$ Line. Sol. Phys.34:151-161, DOI 10.1007/BF00149606

Bell B, Noci G (1976) Intensity of the Fe XV emission line corona, the level of geomagnetic activity, and the velocity of the solar wind. $J$. Geophys. Res.81:4508-4516, DOI 10.1029/JA081i025p04508

Bemporad A (2008) Spectroscopic detection of turbulence in post-cme current sheets. The Astrophysical Journal 689(1):572

Bemporad A, Poletto G, Suess S, Ko YK, Schwadron N, Elliott H, Raymond J (2006) Current sheet evolution in the aftermath of a cme event. The Astrophysical Journal 638(2):1110

Bettarini L, Lapenta G (2010) Spontaneous non-steady magnetic reconnection within the solar environment. Astronomy \& Astrophysics 518:A57

Bhattacharjee A, Huang YM, Yang H, Rogers B (2009) Fast reconnection in highLundquist-number plasmas due to the plasmoid Instability. Physics of Plasmas 16(11):112,102-+, DOI 10.1063/1.3264103, 0906.5599

Birn J, et al (2001) Geospace environmental modeling (gem) magnetic reconnection challenge. J. Geophys. Res.106:3715-3719

Boutry C, Buchlin E, Vial JC, Régnier S (2012) Flows at the Edge of an Active Region: Observation and Interpretation. Astrophys. J.752:13, DOI 10.1088/0004$637 \mathrm{X} / 752 / 1 / 13,1204.1377$

Braginskii SI (1965) Transport Processes in a Plasma. Reviews of Plasma Physics 1:205

Brekke P, Kjeldseth-Moe O, Harrison RA (1997) High-Velocity Flows in an Active Region Loop System Observed with the Coronal Diagnostic Spectrometer (Cds) on SOHO. Sol. Phys.175:511-521, DOI 10.1023/A:1004950330900

Brooks DH, Warren HP (2012) The Coronal Source of Extreme-ultraviolet Line Profile Asymmetries in Solar Active Region Outflows. Astrophys. J. Lett.760:L5, DOI 10.1088/2041-8205/760/1/L5, 1210.1274

Brooks DH, Ugarte-Urra I, Warren HP (2015) Full-Sun observations for identifying the source of the slow solar wind. Nature Communications 6:5947, DOI $10.1038 /$ ncomms6947

Bruno R, Carbone V (2005) The Solar Wind as a Turbulence Laboratory. Living Reviews in Solar Physics 2:4, DOI 10.12942/lrsp-2005-4

Buergi A, Geiss J (1986) Helium and minor ions in the corona and solar wind Dynamics and charge states. Sol. Phys.103:347-383, DOI 10.1007/BF00147835 
Bulanov SV, Sakai J, Syrovatsii SI (1979) Tearing-mode instability in approximately steady mhd configurations. Sov J Plasma Phys 5:157

Burlaga LF, Lazarus AJ (2000) Lognormal distributions and spectra of solar wind plasma fluctuations: Wind 1995-1998. J. Geophys. Res.105:2357-2364, DOI 10.1029/1999JA900442

Burlaga LF, Ness NF, Wang YM, Sheeley NR (2002) Heliospheric magnetic field strength and polarity from 1 to $81 \mathrm{AU}$ during the ascending phase of solar cycle 23. Journal of Geophysical Research (Space Physics) 107:1410, DOI 10.1029/2001JA009217

Cassak PA, Shay MA, Drake JF (2009) Scaling of Sweet-Parker reconnection with secondary islands. Physics of Plasmas 16(12):120,702-+, DOI $10.1063 / 1.3274462$

Chandran BDG, Dennis TJ, Quataert E, Bale SD (2011) Incorporating Kinetic Physics into a Two-fluid Solar-wind Model with Temperature Anisotropy and Low-frequency Alfvén-wave Turbulence. Astrophys. J.743:197, DOI 10.1088/0004-637X/743/2/197, 1110.3029

Chen Y, Esser R, Hu Y (2003) Formation of Minor-Ion Charge States in the Fast Solar Wind: Roles of Differential Flow Speeds of Ions of the Same Element. Astrophys. J.582:467-474, DOI 10.1086/344642

Chen Y, Li X (2004) An Ion-Cyclotron Resonance-driven Three-Fluid Model of the Slow Wind near the Sun. Astrophys. J. Lett.609:L41L44, DOI 10.1086/422581

Chen Y, Esser R, Strachan L, Hu Y (2004) Stagnated Outflow of $\mathrm{O}^{+5}$ Ions in the Source Region of the Slow Solar Wind at Solar Minimum. Astrophys. J.602:415421, DOI 10.1086/380960

Cohen, O., Sokolov, I. V., Roussev, I. I., Arge, C. N., Manchester, W. B., Gombosi, T. I., Frazin, R. A., Park, H., Butala, M. D., Kamalabadi, F., Velli, M.(2007) A Semiempirical Magnetohydrodynamical Model of the Solar Wind. Astrophys. J.654:L163-L166, DOI 10.1086/511154

Cranmer SR (2009) Coronal Holes. Living Reviews in Solar Physics 6:3, DOI 10.12942/lrsp-2009-3, 0909.2847

Cranmer SR, van Ballegooijen AA (2005) On the Generation, Propagation, and Reflection of Alfvén Waves from the Solar Photosphere to the Distant Heliosphere. Astrophys. J. Supp.156:265-293, DOI 10.1086/426507, astro-ph/0410639

Cranmer SR, Field GB, Kohl JL (1999a) Spectroscopic Constraints on Models of Ion Cyclotron Resonance Heating in the Polar Solar Corona and High-Speed Solar Wind. Astrophys. J.518:937-947, DOI 10.1086/307330

Cranmer SR, Kohl JL, Noci G, Antonucci E, Tondello G, Huber MCE, Strachan L, Panasyuk AV, Gardner LD, Romoli M, Fineschi S, Dobrzycka D, Raymond JC, Nicolosi P, Siegmund OHW, Spadaro D, Benna C, Ciaravella A, Giordano S, Habbal SR, Karovska M, Li X, Martin R, Michels JG, Modigliani A, Naletto G, 
O’Neal RH, Pernechele C, Poletto G, Smith PL, Suleiman RM (1999b) An Empirical Model of a Polar Coronal Hole at Solar Minimum. Astrophys. J.511:481501, DOI 10.1086/306675

Cranmer SR, van Ballegooijen AA, Edgar RJ (2007) Self-consistent coronal heating and solar wind acceleration from anisotropic magnetohydrodynamic turbulence. Astrophys J Suppl 171:520, DOI 10.1086/518001, arXiv:astro-ph/0703333

Cranmer SR, Panasyuk AV, Kohl JL (2008) Improved Constraints on the Preferential Heating and Acceleration of Oxygen Ions in the Extended Solar Corona. Astrophys. J.678:1480-1497, DOI 10.1086/586890, 0802.0144

Cranmer SR, Asgari-Targhi M, Miralles MP, Raymond JC, Strachan L, Tian H, Woolsey LN (2014) The Role of Turbulence in Coronal Heating and Solar Wind Expansion. ArXiv e-prints 1412.2307

Crooker NU, Gosling JT, Kahler SW (2002) Reducing heliospheric magnetic flux from coronal mass ejections without disconnection. Journal of Geophysical Research (Space Physics) 107:1028, DOI 10.1029/2001JA000236

Crooker NU, Huang CL, Lamassa SM, Larson DE, Kahler SW, Spence HE (2004) Heliospheric plasma sheets. Journal of Geophysical Research (Space Physics) 109:A03107, DOI 10.1029/2003JA010170

Crooker NU, Appleton EM, Schwadron NA, Owens MJ (2010) Suprathermal electron flux peaks at stream interfaces: Signature of solar wind dynamics or tracer for open magnetic flux transport on the Sun? Journal of Geophysical Research (Space Physics) 115(A14):A11101, DOI 10.1029/2010JA015496

Crooker NU, Antiochos SK, Zhao X, Neugebauer M (2012) Global network of slow solar wind. Journal of Geophysical Research (Space Physics) 117:A04104, DOI 10.1029/2011JA017236

Culhane JL, Brooks DH, van Driel-Gesztelyi L, Démoulin P, Baker D, DeRosa ML, Mandrini CH, Zhao L, Zurbuchen TH (2014) Tracking Solar Active Region Outflow Plasma from Its Source to the Near-Earth Environment. Sol. Phys.289:3799-3816, DOI 10.1007/s11207-014-0551-5, 1405.2949

Cuperman S, Ofman L, Dryer M (1990) Thermally conductive magnetohydrodynamic flows in helmet-streamer coronal structures. Astrophys. J.350:846-855, DOI 10.1086/168436

D'Amicis R, Bruno R (2015) On the Origin of Highly Alfvénic Slow Solar Wind. Astrophys. J.805:84, DOI 10.1088/0004-637X/805/1/84

Daughton W, Roytershteyn V, Albright B, Karimabadi H, Yin L, Bowers KJ (2009) Transition from collisional to kinetic regimes in large-scale reconnection layers. Physical review letters 103(6):065,004

De Pontieu B, McIntosh SW (2010) Quasi-periodic Propagating Signals in the Solar Corona: The Signature of Magnetoacoustic Waves or High-velocity Upflows? Astrophys. J.722:1013-1029, DOI 10.1088/0004-637X/722/2/1013, 1008.5300 
De Pontieu B, McIntosh SW, Hansteen VH, Schrijver CJ (2009) Observing the Roots of Solar Coronal Heating in the Chromosphere. Astrophys. J. Lett.701:L1L6, DOI 10.1088/0004-637X/701/1/L1, 0906.5434

Del Zanna G (2008) Flows in active region loops observed by Hinode EIS. Astron. Astrophys.481:L49-L52, DOI 10.1051/0004-6361:20079087

Del Zanna G, Aulanier G, Klein KL, Török T (2011) A single picture for solar coronal outflows and radio noise storms. Astron. Astrophys.526:A137, DOI $10.1051 / 0004-6361 / 201015231$

Doschek GA, Warren HP, Mariska JT, Muglach K, Culhane JL, Hara H, Watanabe T (2008) Flows and Nonthermal Velocities in Solar Active Regions Observed with the EUV Imaging Spectrometer on Hinode: A Tracer of Active Region Sources of Heliospheric Magnetic Fields? Astrophys. J.686:1362-1371, DOI 10.1086/591724, 0807.2860

Edmondson JK, Lynch BJ, Antiochos SK, De Vore CR, Zurbuchen TH (2009) Reconnection-Driven Dynamics of Coronal-Hole Boundaries. Astrophys. J.707:1427-1437, DOI 10.1088/0004-637X/707/2/1427

Edmondson JK, Antiochos SK, DeVore CR, Lynch BJ, Zurbuchen TH (2010) Interchange Reconnection and Coronal Hole Dynamics. Astrophys. J.714:517531, DOI 10.1088/0004-637X/714/1/517

Edwards SJ, Parnell CE, J HL, L CJ, H BD (2015) A comparison of global magnetic field skeletons and active region upflows. Sol. Phys., in press

Einaudi G, Boncinelli P, Dahlburg RB, Karpen JT (1999) Formation of the slow solar wind in a coronal streamer. Journal of Geophysical Research: Space Physics (1978-2012) 104(A1):521-534

Einaudi G, Chibbaro S, Dahlburg RB, Velli M (2001) Plasmoid formation and acceleration in the solar streamer belt. The Astrophysical Journal 547(2):1167

Endeve E, Leer E (2001) Coronal heating and solar wind acceleration; gyrotropic electron-proton solar wind. Sol. Phys.200:235-250, DOI 10.1023/A:1010313719194

Endeve E, Holzer TE, Leer E (2004) Helmet Streamers Gone Unstable: Two-Fluid Magnetohydrodynamic Models of the Solar Corona. Astrophys. J.603:307-321, DOI $10.1086 / 381239$

Eriksson S, Newman D, Lapenta G, Angelopoulos V (2014) On the signatures of magnetic islands and multiple $\mathrm{x}$-lines in the solar wind as observed by artemis and wind. Plasma Physics and Controlled Fusion 56(6):064,008

Esser R, Edgar RJ (2001) Differential Flow Speeds of Ions of the Same Element: Effects on Solar Wind Ionization Fractions. Astrophys. J.563:1055-1062, DOI $10.1086 / 323987$

Eyink GL, Lazarian A, Vishniac ET (2011) Fast Magnetic Reconnection and Spontaneous Stochasticity. Astrophys. J.743:51, DOI 10.1088/0004-637X/743/1/51, 1103.1882 
Fazakerley A, Harra L, van Driel-Gesztelyi L (2015) An investigation of the sources of Earth-directed solar wind during Carrington rotation 2053. Sol. Phys., submitted

Fineschi S, Gardner LD, Kohl JL, Romoli M, Noci GC (1998) Grating stray light analysis and control in the UVCS/SOHO. In: Fineschi S (ed) X-Ray and Ultraviolet Spectroscopy and Polarimetry II, Society of Photo-Optical Instrumentation Engineers (SPIE) Conference Series, vol 3443, pp 67-74

Fisk LA (2003) Acceleration of the solar wind as a result of the reconnection of open magnetic flux with coronal loops. Journal of Geophysical Research (Space Physics) 108:1157, DOI 10.1029/2002JA009284

Fisk LA, Schwadron NA (2001) Origin of the Solar Wind: Theory. Sp. Sci. Rev.97:21-33

Fisk LA, Zhao L (2009) The heliospheric magnetic field and the solar wind during the solar cycle. In: Gopalswamy N, Webb DF (eds) IAU Symposium, IAU Symposium, vol 257, pp 109-120, DOI 10.1017/S1743921309029160

Fisk LA, Schwadron NA, Zurbuchen TH (1998) On the Slow Solar Wind. Sp. Sci. Rev.86:51-60, DOI 10.1023/A:1005015527146

Frazin RA, Cranmer SR, Kohl JL (2003) Empirically Determined Anisotropic Velocity Distributions and Outflows of $\mathrm{O}^{5+}$ Ions in a Coronal Streamer at Solar Minimum. Astrophys. J.597:1145-1157, DOI 10.1086/378558

Fu H, Li B, Li X, Huang Z, Mou C, Jiao F, Xia L (2015) Coronal Sources and In Situ Properties of the Solar Winds Sampled by ACE During 1999 - 2008. Sol. Phys.290:1399-1415, DOI 10.1007/s11207-015-0689-9, 1505.00407

Geiss J, Gloeckler G, von Steiger R (1995a) Origin of the Solar Wind From Composition Data. Sp. Sci. Rev.72:49-60, DOI 10.1007/BF00768753

Geiss J, Gloeckler G, von Steiger R (1995b) Origin of the Solar Wind From Composition Data. Sp. Sci. Rev.72:49-60, DOI 10.1007/BF00768753

Gloeckler G, Geiss J, Balsiger H, Bedini P, Cain JC, Fischer J, Fisk LA, Galvin AB, Gliem F, Hamilton DC, Hollweg JV, Ipavich FM, Joos R, Livi S, Lundgren RA, Mall U, McKenzie JF, Ogilvie KW, Ottens F, Rieck W, Tums EO, von Steiger R, Weiss W, Wilken B (1992) The Solar Wind Ion Composition Spectrometer. Astron. Astrophys. Supp.92:267-289

Hansteen VH, Leer E (1995) Coronal heating, densities, and temperatures and solar wind acceleration. J. Geophys. Res.100:21,577-21,594, DOI 10.1029/95JA02300

Hansteen VH, Velli M (2012) Solar Wind Models from the Chromosphere to 1 AU. Sp. Sci. Rev.172:89-121, DOI 10.1007/s11214-012-9887-z

Hansteen VH, Hara H, De Pontieu B, Carlsson M (2010) On Redshifts and Blueshifts in the Transition Region and Corona. Astrophys. J.718:1070-1078, DOI 10.1088/0004-637X/718/2/1070, 1001.4769

Harra LK, Sakao T, Mandrini CH, Hara H, Imada S, Young PR, van DrielGesztelyi L, Baker D (2008) Outflows at the Edges of Active Regions: Con- 
tribution to Solar Wind Formation? Astrophys. J. Lett.676:L147-L150, DOI $10.1086 / 587485$

He JS, Marsch E, Tu CY, Guo LJ, Tian H (2010) Intermittent outflows at the edge of an active region - a possible source of the solar wind? Astron. Astrophys.516:A14, DOI 10.1051/0004-6361/200913712

Hollweg JV, Isenberg PA (2002) Generation of the fast solar wind: A review with emphasis on the resonant cyclotron interaction. Journal of Geophysical Research (Space Physics) 107:1147, DOI 10.1029/2001JA000270

Huang YM, Bhattacharjee A (2010) Scaling laws of resistive magnetohydrodynamic reconnection in the high-Lundquist-number, plasmoid-unstable regime. Physics of Plasmas 17(6):062,104-+, DOI 10.1063/1.3420208, 1003.5951

Hundhausen AJ (1972) Coronal Expansion and Solar Wind. Physics and Chemistry in Space 5, DOI 10.1007/978-3-642-65414-5

Ji H, Daughton W (2011) Phase diagram for magnetic reconnection in heliophysical, astrophysical, and laboratory plasmas. Physics of Plasmas 18(11):111,207, DOI 10.1063/1.3647505, 1109.0756

Jiao F, Xia L, Li B, Huang Z, Li X, Chandrashekhar K, Mou C, Fu H (2015) Sources of Quasi-periodic Propagating Disturbances above a Solar Polar Coronal Hole. Astrophys. J. Lett.809:L17, DOI 10.1088/2041-8205/809/1/L17, 1507.08440

Jones SI, Davila JM (2009) Localized Plasma Density Enhancements Observed in STEREO COR1. Astrophys. J.701:1906-1910, DOI 10.1088/0004$637 \mathrm{X} / 701 / 2 / 1906$

Karimabadi H, Daughton W, Quest KB (2005) Antiparallel versus component merging at the magnetopause: Current bifurcation and intermittent reconnection. Journal of Geophysical Research: Space Physics (1978-2012) 110(A3)

Karimabadi H, Daughton W, Scudder J (2007) Multi-scale structure of the electron diffusion region. Geophysical research letters 34(13)

Kasper JC, Stevens ML, Korreck KE, Maruca BA, Kiefer KK, Schwadron NA, Lepri ST (2012) Evolution of the Relationships between Helium Abundance, Minor Ion Charge State, and Solar Wind Speed over the Solar Cycle. Astrophys. J.745:162, DOI 10.1088/0004-637X/745/2/162

Kasper JC, Maruca BA, Stevens ML, Zaslavsky A (2013) Sensitive Test for Ion-Cyclotron Resonant Heating in the Solar Wind. Physical Review Letters 110(9):091102, DOI 10.1103/PhysRevLett.110.091102

Klimchuk JA (2012) The role of type II spicules in the upper solar atmosphere. Journal of Geophysical Research (Space Physics) 117:A12102, DOI 10.1029/2012JA018170, 1207.7048

Knoll D, Chacón L (2002) Magnetic reconnection in the two-dimensional kelvinhelmholtz instability. Physical review letters 88(21):215,003 
Ko YK, Fisk LA, Gloeckler G, Geiss J (1996) Limitations on suprathermal tails of electrons in the lower solar corona. Geophys. Res. Lett.23:2785-2788, DOI 10.1029/96GL02449

Ko YK, Fisk LA, Geiss J, Gloeckler G, Guhathakurta M (1997) An Empirical Study of the Electron Temperature and Heavy Ion Velocities in the South Polar Coronal Hole. Sol. Phys.171:345-361

Ko YK, Geiss J, Gloeckler G (1998) On the differential ion velocity in the inner solar corona and the observed solar wind ionic charge states. J. Geophys. Res.103:14,539-14,546, DOI 10.1029/98JA00763

Ko YK, Raymond JC, Li J, Ciaravella A, Michels J, Fineschi S, Wu R (2002) Solar and Heliospheric Observatory Ultraviolet Coronagraph Spectrometer and Yohkoh Soft X-Ray Telescope Observations of the High-Temperature Corona above an Active Region Complex. Astrophys. J.578:979-995, DOI $10.1086 / 342616$

Ko YK, Li J, Riley P, Raymond JC (2008) Large-Scale Coronal Density and Abundance Structures and Their Association with Magnetic Field Structure. Astrophys. J.683:1168-1179, DOI 10.1086/589873

Ko YK, Muglach K, Wang YM, Young PR, Lepri ST (2014) Temporal Evolution of Solar Wind Ion Composition and their Source Coronal Holes during the Declining Phase of Cycle 23. I. Low-latitude Extension of Polar Coronal Holes. Astrophys. J.787:121, DOI 10.1088/0004-637X/787/2/121

Kohl JL, Noci G, Antonucci E, Tondello G, Huber MCE, Gardner LD, Nicolosi P, Strachan L, Fineschi S, Raymond JC, Romoli M, Spadaro D, Panasyuk A, Siegmund OHW, Benna C, Ciaravella A, Cranmer SR, Giordano S, Karovska M, Martin R, Michels J, Modigliani A, Naletto G, Pernechele C, Poletto G, Smith PL (1997) First Results from the SOHO Ultraviolet Coronagraph Spectrometer. Sol. Phys.175:613-644, DOI 10.1023/A:1004903206467

Kohl JL, Noci G, Antonucci E, Tondello G, Huber MCE, Cranmer SR, Strachan L, Panasyuk AV, Gardner LD, Romoli M, Fineschi S, Dobrzycka D, Raymond JC, Nicolosi P, Siegmund OHW, Spadaro D, Benna C, Ciaravella A, Giordano S, Habbal SR, Karovska M, Li X, Martin R, Michels JG, Modigliani A, Naletto G, O'Neal RH, Pernechele C, Poletto G, Smith PL, Suleiman RM (1998) UVCS/SOHO Empirical Determinations of Anisotropic Velocity Distributions in the Solar Corona. Astrophys. J. Lett.501:L127-L131, DOI 10.1086/311434

Kohl JL, Noci G, Cranmer SR, Raymond JC (2006) Ultraviolet spectroscopy of the extended solar corona. The Astronomy and Astrophysics Review 13:31-157, DOI 10.1007/s00159-005-0026-7

Kopp RA, Holzer TE (1976) Dynamics of coronal hole regions. I - Steady polytropic flows with multiple critical points. Sol. Phys.49:43-56, DOI 10.1007/BF00221484

Kovalenko VA (1981) Energy balance of the corona and the origin of quasi-stationary high-speed solar wind streams. Sol. Phys.73:383-403, DOI 10.1007/BF00151689 
Laming JM (2004) A Unified Picture of the First Ionization Potential and Inverse First Ionization Potential Effects. Astrophys. J.614:1063-1072, DOI 10.1086/423780, astro-ph/0405230

Laming JM (2009) Non-Wkb Models of the First Ionization Potential Effect: Implications for Solar Coronal Heating and the Coronal Helium and Neon Abundances. Astrophys. J.695:954-969, DOI 10.1088/0004-637X/695/2/954, 0901.3350

Laming JM (2015) The FIP and Inverse FIP Effects in Solar and Stellar Coronae. Living Reviews in Solar Physics 12, DOI 10.1007/lrsp-20152, 1504.08325

Laming JM, Lepri ST (2007) Ion Charge States in the Fast Solar Wind: New Data Analysis and Theoretical Refinements. Astrophys. J.660:1642-1652, DOI 10.1086/513505, astro-ph/0702131

Landi E, Testa P (2014) The Temperature of Quiescent Streamers during Solar Cycles 23 and 24. Astrophys. J.787:33, DOI 10.1088/0004-637X/787/1/33

Landi E, Alexander RL, Gruesbeck JR, Gilbert JA, Lepri ST, Manchester WB, Zurbuchen TH (2012a) Carbon Ionization Stages as a Diagnostic of the Solar Wind. Astrophys. J.744:100, DOI 10.1088/0004-637X/744/2/100

Landi E, Gruesbeck JR, Lepri ST, Zurbuchen TH (2012b) New Solar Wind Diagnostic Using Both in Situ and Spectroscopic Measurements. Astrophys. J.750:159, DOI 10.1088/0004-637X/750/2/159

Landi E, Gruesbeck JR, Lepri ST, Zurbuchen TH, Fisk LA (2012c) Charge State Evolution in the Solar Wind. II. Plasma Charge State Composition in the Inner Corona and Accelerating Fast Solar Wind. Astrophys. J.761:48, DOI 10.1088/0004-637X/761/1/48

Landi E, Gruesbeck JR, Lepri ST, Zurbuchen TH, Fisk LA (2012d) Charge State Evolution in the Solar Wind. Radiative Losses in Fast Solar Wind Plasmas. Astrophys. J. Lett.758:L21, DOI 10.1088/2041-8205/758/1/L21

Lapenta G (2008) Self-Feeding Turbulent Magnetic Reconnection on Macroscopic Scales. Physical Review Letters 100(23):235,001-+, DOI 10.1103/PhysRevLett.100.235001, 0805.0426

Lapenta G, Knoll D (2005a) Effect of a Converging Flow at the Streamer Cusp on the Genesis of the Slow Solar Wind. Astrophys J 624:1049-1056

Lapenta G, Knoll D (2005b) Effect of a converging flow at the streamer cusp on the genesis of the slow solar wind. The Astrophysical Journal 624(2):1049

Lapenta G, Lazarian A (2012) Achieving fast reconnection in resistive MHD models via turbulent means. Nonlinear Processes in Geophysics 19:251-263, DOI 10.5194/npg-19-251-2012, 1110.0089

Lapenta G, Restante AL (2008) Blob formation and acceleration in the solar wind: role of converging flows and viscosity. Annales Geophysicae 26:3049-3060, DOI 10.5194/angeo-26-3049-2008, 0710.2702 
Lazarian A, Vishniac ET (1999) Reconnection in a Weakly Stochastic Field. Astrophys. J.517:700-718, DOI 10.1086/307233, arXiv:astro-ph/9811037

Le Chat G, Issautier K, Meyer-Vernet N (2012) The Solar Wind Energy Flux. Sol. Phys.279:197-205, DOI 10.1007/s11207-012-9967-y, 1203.1316

Leer E, Holzer TE (1980) Energy addition in the solar wind. J. Geophys. Res.85:4681-4688, DOI 10.1029/JA085iA09p04681

Leer E, Holzer TE, Fla T (1982) Acceleration of the solar wind. Sp. Sci. Rev.33:161-200, DOI 10.1007/BF00213253

Lepri ST, Landi E, Zurbuchen TH (2013) Solar Wind Heavy Ions over Solar Cycle 23: ACE/SWICS Measurements. Astrophys. J.768:94, DOI 10.1088/0004$637 \mathrm{X} / 768 / 1 / 94$

Levine RH, Altschuler MD, Harvey JW (1977) Solar sources of the interplanetary magnetic field and solar wind. J. Geophys. Res.82:1061-1065, DOI 10.1029/JA082i007p01061

Li B, Li X, Hu YQ, Habbal SR (2004) A two-dimensional Alfvén wave-driven solar wind model with proton temperature anisotropy. Journal of Geophysical Research (Space Physics) 109:A07103, DOI 10.1029/2003JA010313

Li B, Li X, Labrosse N (2006) A global 2.5-dimensional three fluid solar wind model with alpha particles. Journal of Geophysical Research (Space Physics) 111:A08106, DOI 10.1029/2005JA011303

Li B, Xia LD, Chen Y (2011) Solar winds along curved magnetic field lines. Astron. Astrophys.529:A148, DOI 10.1051/0004-6361/201116668, 1103.5211

Li J, Raymond JC, Acton LW, Kohl JL, Romoli M, Noci G, Naletto G (1998) Physical Structure of a Coronal Streamer in the Closed-Field Region as Observed from UVCS/SOHO and SXT/Yohkoh. Astrophys. J.506:431-438, DOI $10.1086 / 306244$

Li X, Esser R, Habbal SR, Hu YQ (1997) Influence of heavy ions on the high-speed solar wind. J. Geophys. Res.102:17,419-17,432, DOI 10.1029/97JA01448

Lie-Svendsen Ø, Hansteen VH, Leer E, Holzer TE (2002) The Effect of Transition Region Heating on the Solar Wind from Coronal Holes. Astrophys. J.566:562576, DOI 10.1086/337990

Liewer PC, Neugebauer M, Zurbuchen T (2004) Characteristics of active-region sources of solar wind near solar maximum. Sol. Phys.223:209-229, DOI 10.1007/s11207-004-1105-z

Linker, J. A., van Hoven, G., Schnack, D. D. (1990) A three-dimensional simulation of a coronal streamer. Geophys. Res. Lett.17:2281-2284, DOI 10.1029/GL017i013p02281

Linker JA, Lionello R, Mikić Z, Titov VS, Antiochos SK (2011) The Evolution of Open Magnetic Flux Driven by Photospheric Dynamics. Astrophys. J.731:110, DOI 10.1088/0004-637X/731/2/110 
Lionello R, Linker JA, Mikić Z (2001) Including the Transition Region in Models of the Large-Scale Solar Corona. Astrophys. J.546:542-551, DOI 10.1086/318254

Lionello R, Riley P, Linker JA, Mikić Z (2005) The effects of differential rotation on the magnetic structure of the solar corona: magnetohydrodynamic simulations. Astrophys J 625:463, DOI 10.1086/429268

Lionello R, Linker JA, Mikić Z (2009) Multispectral Emission of the Sun During the First Whole Sun Month: Magnetohydrodynamic Simulations. Astrophys J 690:902-912, DOI 10.1088/0004-637X/690/1/902

Lionello R, Velli M, Linker JA, Mikić Z (2013) Integrating physics-based coronal heating and solar wind acceleration in a global MHD model. In: Zank GP, Borovsky J, Bruno R, Cirtain J, Cranmer S, Elliott H, Giacalone J, Gonzalez W, Li G, Marsch E, Moebius E, Pogorelov N, Spann J, Verkhoglyadova O (eds) American Institute of Physics Conference Series, American Institute of Physics Conference Series, vol 1539, pp 30-33, DOI 10.1063/1.4810982

Loureiro NF, Schekochihin AA, Cowley SC (2007) Instability of current sheets and formation of plasmoid chains. Physics of Plasmas 14(10):100,703-+, DOI 10.1063/1.2783986, arXiv:astro-ph/0703631

Madjarska MS, Wiegelmann T (2009) Coronal hole boundaries evolution at small scales. I. EIT $195 \AA$ and TRACE $171 \AA \AA$ view. Astron. Astrophys.503:991-997, DOI 10.1051/0004-6361/200912066, 0906.2556

Madjarska MS, Doyle JG, van Driel-Gesztelyi L (2004) Evidence of Magnetic Reconnection along Coronal Hole Boundaries. Astrophys. J. Lett.603:L57-L59, DOI $10.1086 / 383030$

Madjarska MS, Huang Z, Doyle JG, Subramanian S (2012) Coronal hole boundaries evolution at small scales. III. EIS and SUMER views. Astron. Astrophys.545:A67, DOI 10.1051/0004-6361/201219516, 1207.1281

Mandrini CH, Nuevo FA, Vásquez AM, Démoulin P, van Driel-Gesztelyi L, Baker D, Culhane JL, Cristiani GD, Pick M (2014) How Can Active Region Plasma Escape into the Solar Wind from Below a Closed Helmet Streamer? Sol. Phys.289:4151-4171, DOI 10.1007/s11207-014-0582-y, 1409.7369

Maneva YG, Ofman L, Viñas A (2015) Relative drifts and temperature anisotropies of protons and $\alpha$ particles in the expanding solar wind: 2.5D hybrid simulations. Astron. Astrophys.578:A85, DOI 10.1051/0004-6361/201424401, 1410.3358

Markidis S, Henri P, Lapenta G, Divin A, Goldman M, Newman D, Eriksson S (2012) Collisionless magnetic reconnection in a plasmoid chain. Nonlinear processes in geophysics 19(1):145-153

Markidis S, Henri P, Lapenta G, Divin A, Goldman M, Newman D, Laure E (2013) Kinetic simulations of plasmoid chain dynamics. Physics of Plasmas (1994-present) 20(8):082,105

Marocchi D, Antonucci E, Giordano S (2001) Oxygen abundance in coronal streamers during solar minimum. Annales Geophysicae 19:135-145, DOI 10.5194/angeo-19-135-2001 
Marsch E (1991) Kinetic Physics of the Solar Wind Plasma. In: Schwenn R, Marsch E (eds) Physics of the Inner Heliosphere II, Springer-Verlag Berlin Heidelberg New York, pp 45-133

Maruca BA, Kasper JC, Bale SD (2011) What Are the Relative Roles of Heating and Cooling in Generating Solar Wind Temperature Anisotropies? Physical Review Letters 107(20):201101, DOI 10.1103/PhysRevLett.107.201101

Maruca BA, Kasper JC, Gary SP (2012) Instability-driven Limits on Helium Temperature Anisotropy in the Solar Wind: Observations and Linear Vlasov Analysis. Astrophys. J.748:137, DOI 10.1088/0004-637X/748/2/137

Matthaeus W, Lamkin S (1986) Turbulent magnetic reconnection. Phys Fluids 29(8):2513-2534, DOI 10.1063/1.866004

McComas DJ, Bame SJ, Barraclough BL, Feldman WC, Funsten HO, Gosling JT, Riley P, Skoug R, Balogh A, Forsyth R, Goldstein BE, Neugebauer M (1998) Ulysses' return to the slow solar wind. Geophys. Res. Lett.25:1-4, DOI 10.1029/97GL03444

McComas DJ, Ebert RW, Elliott HA, Goldstein BE, Gosling JT, Schwadron NA, Skoug RM (2008) Weaker solar wind from the polar coronal holes and the whole Sun. Geophys. Res. Lett.35:L18103, DOI 10.1029/2008GL034896

Mikić Z, Linker JA, Lionello R, Riley P, Titov V (2007) Predicting the Structure of the Solar Corona for the Total Solar Eclipse of March 29, 2006. In: Demircan O, Selam SO, Albayrak B (eds) Solar and Stellar Physics Through Eclipses, Astronomical Society of the Pacific Conference Series, vol 370, p 299

Munro RH, Jackson BV (1977) Physical properties of a polar coronal hole from 2 to 5 solar radii. Astrophys. J.213:874, DOI 10.1086/155220

Nakariakov VM, Ofman L, Arber TD (2000) Nonlinear Dissipative Spherical Alfvén Waves In Solar Coronal Holes. Astron. Astrophys.353:741-748

Neugebauer M, Liewer PC, Smith EJ, Skoug RM, Zurbuchen TH (2002) Sources of the solar wind at solar activity maximum. Journal of Geophysical Research (Space Physics) 107:1488, DOI 10.1029/2001JA000306

Neugebauer M, Liewer PC, Goldstein BE, Zhou X, Steinberg JT (2004) Solar wind stream interaction regions without sector boundaries. Journal of Geophysical Research (Space Physics) 109:A10102, DOI 10.1029/2004J A010456

Noci G, Gavryuseva E (2007) Plasma Outflows in Coronal Streamers. Astrophys. J. Lett.658:L63-L66, DOI 10.1086/513506

Noci G, Kohl JL, Withbroe GL (1987) Solar wind diagnostics from Dopplerenhanced scattering. Astrophys. J.315:706-715, DOI 10.1086/165172

Noci G, Kohl JL, Antonucci E, Tondello G, Huber MCE, Fineschi S, Gardner LD, Korendyke CM, Nicolosi P, Romoli M, Spadaro D, Maccari L, Raymond JC, Siegmund OHW, Benna C, Ciaravella A, Giordano S, Michels J, Modigliani A, Naletto G, Panasyuk A, Pernechele C, Poletto G, Smith PL, Strachan L 
(1997a) The quiescent corona and slow solar wind. In: Wilson A (ed) Fifth SOHO Workshop: The Corona and Solar Wind Near Minimum Activity, ESA Special Publication, vol 404, p 75

Noci G, Kohl JL, Antonucci E, Tondello G, Huber MCE, Fineschi S, Gardner LD, Naletto G, Nicolosi P, Raymond JC, Romoli M, Spadaro D, Siegmund OHW, Benna C, Ciaravella A, Giordano S, Michels J, Modigliani A, Panasyuk A, Pernechele C, Poletto G, Smith PL, Strachan L (1997b) First results from UVCS/SOHO. Advances in Space Research 20:2219-2230, DOI 10.1016/S02731177(97)00895-8

Ofman L (2000) Source regions of the slow solar wind in coronal streamers. Geophys. Res. Lett.27:2885-2888, DOI 10.1029/2000GL000097

Ofman L (2004a) The origin of the slow solar wind in coronal streamers. Advances in Space Research 33:681-688, DOI 10.1016/S0273-1177(03)00235-7

Ofman L (2004b) Three-fluid model of the heating and acceleration of the fast solar wind. Journal of Geophysical Research (Space Physics) 109:A07102, DOI 10.1029/2003JA010221

Ofman L (2010) Wave Modeling of the Solar Wind. Living Reviews in Solar Physics 7:4, DOI 10.12942/lrsp-2010-4

Ofman L, Davila JM (1997) Do First Results from SOHO UVCS Indicate That the Solar Wind Is Accelerated by Solitary Waves? Astrophys. J. Lett.476:L51-L54, DOI 10.1086/310491

Ofman L, Davila JM (1998) Solar wind acceleration by large-amplitude nonlinear waves: Parametric study. J. Geophys. Res.103(A10):23,677-23,690

Ofman L, Kramar M (2010) Modeling the Slow Solar Wind during the Solar Minimum. In: Cranmer SR, Hoeksema JT, Kohl JL (eds) SOHO-23: Understanding a Peculiar Solar Minimum, Astronomical Society of the Pacific Conference Series, vol 428, p 321, 1004.4847

Ofman L, Chen XL, Morrison PJ, Steinolfson RS (1991) Resistive tearing mode instability with shear flow and viscosity. Physics of Fluids B 3:1364-1373, DOI $10.1063 / 1.859701$

Ofman L, Morrison P, Steinolfson R (1993) Nonlinear evolution of resistive tearing mode instability with shear flow and viscosity. Physics of Fluids B: Plasma Physics (1989-1993) 5(2):376-387

Ofman L, Romoli M, Poletto G, Noci G, Kohl JL (1997) Ultraviolet Coronagraph Spectrometer Observations of Density Fluctuations in the Solar Wind. Astrophys. J. Lett.491:L111-L114, DOI 10.1086/311067

Ofman L, Abbo L, Giordano S (2011) Multi-fluid Model of a Streamer at Solar Minimum and Comparison with Observations. Astrophys. J.734:30, DOI 10.1088/0004-637X/734/1/30

Ofman L, Wang TJ, Davila JM (2012) Slow Magnetosonic Waves and Fast Flows in Active Region Loops. Astrophys. J.754:111, DOI 10.1088/0004$637 \mathrm{X} / 754 / 2 / 111,1205.5732$ 
Ofman L, Abbo L, Giordano S (2013) Observations and Models of Slow Solar Wind with $\mathrm{Mg}^{9+}$ Ions in Quiescent Streamers. Astrophys. J.762:18, DOI 10.1088/0004-637X/762/1/18, 1211.1524

Ofman L, Viñas AF, Maneva Y (2014) Two-dimensional hybrid models of $\mathrm{H}^{+}$$\mathrm{He}^{++}$expanding solar wind plasma heating. Journal of Geophysical Research (Space Physics) 119:4223-4238, DOI 10.1002/2013JA019590

Ofman L, Provornikova E, Abbo L, Giordano S (2015) Three-dimensional multifluid model of a coronal streamer belt with a tilted magnetic dipole. Annales Geophysicae 33:47-53, DOI 10.5194/angeo-33-47-2015

Owocki SP, Scudder JD (1983) The effect of a non-Maxwellian electron distribution on oxygen and iron ionization balances in the solar corona. Astrophys. J.270:758-768, DOI 10.1086/161167

Owocki SP, Holzer TE, Hundhausen AJ (1983) The solar wind ionization state as a coronal temperature diagnostic. Astrophys. J.275:354-366, DOI 10.1086/161538

Ozak N, Ofman L, Viñas AF (2015) Ion Heating in Inhomogeneous Expanding Solar Wind Plasma: The Role of Parallel and Oblique Ion-cyclotron Waves. Astrophys. J.799:77, DOI 10.1088/0004-637X/799/1/77, 1407.4622

Parker EN (1957) Sweet's Mechanism for Merging Magnetic Fields in Conducting Fluids. J Geophs Res 62:509-520, DOI 10.1029/JZ062i004p00509

Parker EN (1958) Dynamics of the Interplanetary Gas and Magnetic Fields. Astrophys. J.128:664, DOI 10.1086/146579

Peter H (1998) Element fractionation in the solar chromosphere driven by ionization-diffusion processes. Astron. Astrophys.335:691-702

Petschek HE (1964) Magnetic Field Annihilation. NASA Special Publication 50:425

Pinto RF, Brun AS, Rouillard AP (2016) Flux-tube geometry and wind speed during an activity cycle. ArXiv e-prints 1603.09251

Pizzo V (1978) A three-dimensional model of corotating streams in the solar wind. I - Theoretical foundations. J. Geophys. Res.83:55635572, DOI 10.1029/JA083iA12p05563

Poletto G, Suess ST, Biesecker DA, Esser R, Gloeckler G, Ko YK, Zurbuchen TH (2002) Low-latitude solar wind during the Fall 1998 SOHO-Ulysses quadrature. Journal of Geophysical Research (Space Physics) 107:1300, DOI 10.1029/2001JA000275

Priest E, Forbes T (2000) Magnetic Reconnection : MHD Theory and Applications. Cambridge University Press, Cambridge

Rappazzo AF, Velli M, Einaudi G, Dahlburg RB (2005) Diamagnetic and Expansion Effects on the Observable Properties of the Slow Solar Wind in a Coronal Streamer. Astrophys. J.633:474-488, DOI 10.1086/431916, 1002.3325 
Rappazzo AF, Matthaeus WH, Ruffolo D, Servidio S, Velli M (2012) Interchange Reconnection in a Turbulent Corona. Astrophys. J. Lett.758:L14, DOI 10.1088/2041-8205/758/1/L14, 1209.1388

Raymond JC, Kohl JL, Noci G, Antonucci E, Tondello G, Huber MCE, Gardner LD, Nicolosi P, Fineschi S, Romoli M, Spadaro D, Siegmund OHW, Benna C, Ciaravella A, Cranmer S, Giordano S, Karovska M, Martin R, Michels J, Modigliani A, Naletto G, Panasyuk A, Pernechele C, Poletto G, Smith PL, Suleiman RM, Strachan L (1997) Composition of Coronal Streamers from the SOHO Ultraviolet Coronagraph Spectrometer. Sol. Phys.175:645-665, DOI 10.1023/A:1004948423169

Raymond JC, Mazur JE, Allegrini F, Antonucci E, Del Zanna G, Giordano S, Ho G, Ko YK, Landi E, Lazarus A, Parenti S, Poletto G, Reinard A, Rodriguez-Pacheco J, Teriaca L, Wurz P, Zangrilli L (2001) Coronal and solar wind elemental abundances. In: Wimmer-Schweingruber RF (ed) Joint SOHO/ACE workshop "Solar and Galactic Composition", American Institute of Physics Conference Series, vol 598, pp 49-57, DOI 10.1063/1.1433978

Riley P, Luhmann JG (2012) Interplanetary Signatures of Unipolar Streamers and the Origin of the Slow Solar Wind. Solar Phys 277:355-373, DOI $10.1007 / \mathrm{s} 11207-011-9909-0$

Riley P, Linker JA, Mikić Z (2001) An empirically-driven global mhd model of the corona and inner heliosphere. J Geophys Res 106:15,889, DOI 10.1029/2000JA000121

Riley P, Linker JA, Mikić Z, Odstrcil D, Zurbuchen TH, Lario D, Lepping RP (2003) Using an MHD simulation to interpret the global context of a coronal mass ejection observed by two spacecraft. J Geophys Res 108:1272

Riley P, Linker JA, Mikić Z, Lionello R, Ledvina SA, Luhmann JG (2006) A comparison between global solar magnetohydrodynamic and potential field source surface model results. Astrophys J 653:1510, DOI 10.1086/508565

Riley P, Lionello R, Mikić Z, Linker J, Clark E, Lin J, Ko YK (2007) "Bursty" Reconnection Following Solar Eruptions: MHD Simulations and Comparison with Observations. Astrophys J 655:591-597, DOI 10.1086/509913

Riley P, Lionello R, Mikić Z, Linker J (2008) Using Global Simulations to Relate the Three-Part Structure of Coronal Mass Ejections to In Situ Signatures. Astrophys J 672:1221-1227, DOI 10.1086/523893

Sakao T, Kano R, Narukage N, Kotoku J, Bando T, DeLuca EE, Lundquist LL, Tsuneta S, Harra LK, Katsukawa Y, Kubo M, Hara H, Matsuzaki K, Shimojo M, Bookbinder JA, Golub L, Korreck KE, Su Y, Shibasaki K, Shimizu T, Nakatani I (2007) Continuous Plasma Outflows from the Edge of a Solar Active Region as a Possible Source of Solar Wind. Science 318:1585-, DOI 10.1126/science.1147292

Samtaney R, Loureiro NF, Uzdensky DA, Schekochihin AA, Cowley SC (2009) Formation of Plasmoid Chains in Magnetic Reconnection. Physical Review Letters 103(10):105,004-+, DOI 10.1103/PhysRevLett.103.105004, 0903.0542 
Schrijver CJ, Title AM, van Ballegooijen AA, Hagenaar HJ, Shine RA (1997) Sustaining the Quiet Photospheric Network: The Balance of Flux Emergence, Fragmentation, Merging, and Cancellation. Astrophys. J.487:424-436

Schwadron NA, Fisk LA, Zurbuchen TH (1999) Elemental Fractionation in the Slow Solar Wind. Astrophys. J.521:859-867, DOI 10.1086/307575

Schwadron NA, Smith CW, Spence HE, Kasper JC, Korreck K, Stevens ML, Maruca BA, Kiefer KK, Lepri ST, McComas D (2011) Coronal Electron Temperature from the Solar Wind Scaling Law throughout the Space Age. Astrophys. J.739:9, DOI 10.1088/0004-637X/739/1/9

Schwenn R (2006) Solar Wind Sources and Their Variations Over the Solar Cycle. Sp. Sci. Rev.124:51-76, DOI 10.1007/s11214-006-9099-5

Shearer P, von Steiger R, Raines JM, Lepri ST, Thomas JW, Gilbert JA, Landi E, Zurbuchen TH (2014) The Solar Wind Neon Abundance Observed with ACE/SWICS and Ulysses/SWICS. Astrophys. J.789:60, DOI 10.1088/0004$637 \mathrm{X} / 789 / 1 / 60$

Sheeley NR, Wang YM, Hawley SH, Brueckner GE, Dere KP, Howard RA, Koomen MJ, Korendyke CM, Michels DJ, Paswaters SE, Socker DG, St Cyr OC, Wang D, Lamy PL, Llebaria A, Schwenn R, Simnett GM, Plunkett S, Biesecker DA (1997) Measurements of Flow Speeds in the Corona Between 2 and $30 \mathrm{R}_{\odot}$. Astrophys. J.484:472-478

Sheeley NR Jr, Lee DDH, Casto KP, Wang YM, Rich NB (2009) The Structure of Streamer Blobs. Astrophys. J.694:1471, DOI 10.1088/0004-637X/694/2/1471

Skender M, Lapenta G (2010) On the instability of a quasiequilibrium current sheet and the onset of impulsive bursty reconnection. Phys Plasmas 17:022,905

Slemzin V, Harra L, Urnov A, Kuzin S, Goryaev F, Berghmans D (2013) Signatures of Slow Solar Wind Streams from Active Regions in the Inner Corona. Sol. Phys.286:157-184, DOI 10.1007/s11207-012-0004-y, 1203.6756

Song HQ, Chen Y, Liu K, Feng SW, Xia LD (2009) Quasi-Periodic Releases of Streamer Blobs and Velocity Variability of the Slow Solar Wind near the Sun. Sol. Phys.258:129-140, DOI 10.1007/s11207-009-9411-0, 0907.0819

Spadaro D, Susino R, Ventura R, Vourlidas A, Landi E (2007) Physical parameters of a mid-latitude streamer during the declining phase of the solar cycle. Astron. Astrophys.475:707-715, DOI 10.1051/0004-6361:20077873

Strachan L, Suleiman R, Panasyuk AV, Biesecker DA, Kohl JL (2002) Empirical Densities, Kinetic Temperatures, and Outflow Velocities in the Equatorial Streamer Belt at Solar Minimum. Astrophys. J.571:1008-1014, DOI 10.1086/339984

Strachan L, Panasyuk AV, Kohl JL, Lamy P (2012) The Evolution of Plasma Parameters on a Coronal Source Surface at $2.3 \mathrm{R} \odot$ during Solar Minimum. Astrophys. J.745:51, DOI 10.1088/0004-637X/745/1/51, 1111.1206

Subramanian S, Madjarska MS, Doyle JG (2010) Coronal hole boundaries evolution at small scales. II. XRT view. Can small-scale outflows at CHBs be a 
source of the slow solar wind. Astron. Astrophys.516:A50, DOI 10.1051/0004$6361 / 200913624,1002.1675$

Suess ST (1979) Models of coronal hole flows. Sp. Sci. Rev.23:159-200, DOI 10.1007/BF00173809

Suess ST, Wang AH, Wu ST (1996) Volumetric heating in coronal streamers. J. Geophys. Res.101:19,957-19,966, DOI 10.1029/96JA01458

Suess ST, Wang AH, Wu ST, Nerney SF (1999) Streamer Evaporation. Sp. Sci. Rev.87:323-326, DOI 10.1023/A:1005149929192

Suess ST, Ko YK, von Steiger R, Moore RL (2009) Quiescent current sheets in the solar wind and origins of slow wind. Journal of Geophysical Research (Space Physics) 114:A04103, DOI 10.1029/2008JA013704

Susino R, Ventura R, Spadaro D, Vourlidas A, Landi E (2008) Physical parameters along the boundaries of a mid-latitude streamer and in its adjacent regions. Astron. Astrophys.488:303-310, DOI 10.1051/0004-6361:200809713

Suzuki TK (2004) Coronal heating and acceleration of the high/low-speed solar wind by fast/slow MHD shock trains. MNRAS 349:1227-1239

Sweet PA (1958) The Neutral Point Theory of Solar Flares. In: Lehnert B (ed) Electromagnetic Phenomena in Cosmical Physics, IAU Symposium, vol 6, p 123

Teriaca L, Banerjee D, Doyle JG (1999) SUMER observations of Doppler shift in the quiet Sun and in an active region. Astron. Astrophys.349:636-648

Tian H, McIntosh SW, De Pontieu B (2011) The Spectroscopic Signature of Quasiperiodic Upflows in Active Region Timeseries. Astrophys. J. Lett.727:L37, DOI 10.1088/2041-8205/727/2/L37, 1012.5112

Tian H, McIntosh SW, Wang T, Ofman L, De Pontieu B, Innes DE, Peter H (2012) Persistent Doppler Shift Oscillations Observed with Hinode/EIS in the Solar Corona: Spectroscopic Signatures of Alfvénic Waves and Recurring Upflows. Astrophys. J.759:144, DOI 10.1088/0004-637X/759/2/144, 1209.5286

Timothy AF, Krieger AS, Vaiana GS (1975) The structure and evolution of coronal holes. Sol. Phys.42:135-156, DOI 10.1007/BF00153291

Titov VS, Hornig G, Démoulin P (2002) Theory of magnetic connectivity in the solar corona. Journal of Geophysical Research (Space Physics) 107:1164, DOI 10.1029/2001JA000278

Titov VS, Mikić Z, Linker JA, Lionello R, Antiochos SK (2011) Magnetic Topology of Coronal Hole Linkages. Astrophys. J.731:111, DOI 10.1088/0004637X/731/2/111, 1011.0009

Titov VS, Mikic Z, Török T, Linker JA, Panasenco O (2012) 2010 August 1-2 Sympathetic Eruptions. I. Magnetic Topology of the Source-surface Background Field. Astrophys. J.759:70, DOI 10.1088/0004-637X/759/1/70, 1209.5797

Tokumaru M, Kojima M, Fujiki K (2010) Solar cycle evolution of the solar wind speed distribution from 1985 to 2008. Journal of Geophysical Research (Space Physics) 115:A04102, DOI 10.1029/2009JA014628 
Ugarte-Urra I, Warren HP (2011) Temporal Variability of Active Region Outflows. Astrophys. J.730:37, DOI 10.1088/0004-637X/730/1/37, 1008.4730

Uzdensky DA, Loureiro DA, Schekochihin AA (2010) Fast Magnetic Reconnection in the Plasmoid-Dominated Regime. Phys Rev Lett 105:235,002

Uzzo M, Ko YK, Raymond JC, Wurz P, Ipavich FM (2003) Elemental Abundances for the 1996 Streamer Belt. Astrophys. J.585:1062-1072, DOI 10.1086/346132

van der Holst B, Sokolov IV, Meng X, Jin M, Manchester WB IV, Tóth G, Gombosi TI (2014) Alfvén Wave Solar Model (AWSoM): Coronal Heating. Astrophys. J.782:81, DOI 10.1088/0004-637X/782/2/81, 1311.4093

van Driel-Gesztelyi L, Culhane JL, Baker D, Démoulin P, Mandrini CH, DeRosa ML, Rouillard AP, Opitz A, Stenborg G, Vourlidas A, Brooks DH (2012) Magnetic Topology of Active Regions and Coronal Holes: Implications for Coronal Outflows and the Solar Wind. Sol. Phys.281:237-262, DOI 10.1007/s11207-0120076-8

Viall NM, Vourlidas A (2015) Periodic Density Structures and the Origin of the Slow Solar Wind. Astrophys. J.807:176, DOI 10.1088/0004-637X/807/2/176

von Steiger R (2008) The solar wind throughout the solar cycle, Praxis Publishing Ltd, Chichester, UK, p 41. DOI 10.1007/978-3-540-74302-63

von Steiger R, Geiss J (1989) Supply of fractionated gases to the corona. Astron. Astrophys.225:222-238

von Steiger R, Zurbuchen TH (2006) Kinetic properties of heavy solar wind ions from Ulysses-SWICS. Geophys. Res. Lett.33:L09103, DOI 10.1029/2005GL024998

von Steiger R, Zurbuchen TH (2011) Polar coronal holes during the past solar cycle: Ulysses observations. Journal of Geophysical Research (Space Physics) 116:A01105, DOI 10.1029/2010JA015835

\section{von Steiger R, Zurbuchen TH (2016) Solar Metallicity Derived from in situ Solar Wind Composition. Astrophys. J.816:13, DOI 10.3847/0004-637X/816/1/13}

von Steiger R, Schwadron NA, Fisk LA, Geiss J, Gloeckler G, Hefti S, Wilken B, Wimmer-Schweingruber RF, Zurbuchen TH (2000) Composition of quasistationary solar wind flows from Ulysses/Solar Wind Ion Composition Spectrometer. J. Geophys. Res.105:27,217-27,238, DOI 10.1029/1999JA000358

von Steiger R, Zurbuchen TH, McComas DJ (2010) Oxygen flux in the solar wind: Ulysses observations. Geophys. Res. Lett.37:L22101, DOI 10.1029/2010GL045389

Wan W, Lapenta G (2008a) Evolutions of non-steady-state magnetic reconnection. Physics of Plasmas (1994-present) 15(10):102,302

Wan W, Lapenta G (2008b) Micro-macro coupling in plasma self-organization processes during island coalescence. Physical review letters 100(3):035,004 
Wang T, Ofman L, Davila JM (2013) Three-dimensional Magnetohydrodynamic Modeling of Propagating Disturbances in Fan-like Coronal Loops. Astrophys. J. Lett.775:L23, DOI 10.1088/2041-8205/775/1/L23, 1308.0282

Wang TJ, Ofman L, Davila JM (2009a) Propagating Slow Magnetoacoustic Waves in Coronal Loops Observed by Hinode/EIS. Astrophys. J.696:1448-1460, DOI 10.1088/0004-637X/696/2/1448, 0902.4480

Wang YM (2010) On the Relative Constancy of the Solar Wind Mass Flux at 1 AU. Astrophys. J. Lett.715:L121-L127, DOI 10.1088/2041-8205/715/2/L121

Wang YM, Sheeley NR Jr (1990) Solar wind speed and coronal flux-tube expansion. Astrophys. J.355:726-732, DOI 10.1086/168805

Wang YM, Sheeley NR Jr, Walters JH, Brueckner GE, Howard RA, Michels DJ, Lamy PL, Schwenn R, Simnett GM (1998) Origin of Streamer Material in the Outer Corona. Astrophys. J. Lett.498:L165-L168, DOI 10.1086/311321

Wang YM, Sheeley NR, Socker DG, Howard RA, Rich NB (2000) The dynamical nature of coronal streamers. J. Geophys. Res.105:25,133-25,142, DOI 10.1029/2000JA000149

Wang YM, Biersteker JB, Sheeley NR Jr, Koutchmy S, Mouette J, Druckmüller M (2007a) The Solar Eclipse of 2006 and the Origin of Raylike Features in the White-Light Corona. Astrophys. J.660:882-892, DOI 10.1086/512480

Wang YM, Sheeley NR Jr, Rich NB (2007b) Coronal Pseudostreamers. Astrophys. J.658:1340-1348, DOI 10.1086/511416

Wang YM, Ko YK, Grappin R (2009b) Slow Solar Wind from Open Regions with Strong Low-Coronal Heating. Astrophys. J.691:760-769, DOI 10.1088/0004$637 \mathrm{X} / 691 / 1 / 760$

Wang YM, Grappin R, Robbrecht E, Sheeley NR Jr (2012) On the Nature of the Solar Wind from Coronal Pseudostreamers. Astrophys. J.749:182, DOI 10.1088/0004-637X/749/2/182

Warren HP, Ugarte-Urra I, Young PR, Stenborg G (2011) The Temperature Dependence of Solar Active Region Outflows. Astrophys. J.727:58, DOI 10.1088/0004-637X/727/1/58, 1008.2696

Weberg MJ, Zurbuchen TH, Lepri ST (2012) ACE/SWICS Observations of Heavy Ion Dropouts within the Solar Wind. Astrophys. J.760:30, DOI 10.1088/0004$637 \mathrm{X} / 760 / 1 / 30$

Weberg MJ, Lepri ST, Zurbuchen TH (2015) Coronal Sources, Elemental Fractionation, and Release Mechanisms of Heavy Ion Dropouts in the Solar Wind. Astrophys. J.801:99, DOI 10.1088/0004-637X/801/2/99

Wenzel KP, Marsden RG, Page DE, Smith EJ (1992) The ULYSSES Mission. Astron. Astrophys. Supp.92:207

Widing KG, Feldman U (2001) On the Rate of Abundance Modifications versus Time in Active Region Plasmas. Astrophys. J.555:426434, DOI 10.1086/321482 
Withbroe GL (1988) The temperature structure, mass, and energy flow in the corona and inner solar wind. Astrophys. J.325:442-467, DOI 10.1086/166015

Withbroe GL, Kohl JL, Weiser H, Munro RH (1982) Probing the solar wind acceleration region using spectroscopic techniques. Sp. Sci. Rev.33:17-52, DOI 10.1007/BF00213247

Zhao L, Landi E (2014) Polar and Equatorial Coronal Hole Winds at Solar Minima: From the Heliosphere to the Inner Corona. Astrophys. J.781:110, DOI 10.1088/0004-637X/781/2/110

Zhao L, Zurbuchen TH, Fisk LA (2009) Global distribution of the solar wind during solar cycle 23: ACE observations. Geophys. Res. Lett.36:L14104, DOI 10.1029/2009GL039181

Zirker JB (ed) (1977) Coronal holes and high speed wind streams: a monograph from Skylab solar workshop I.

Zurbuchen TH (2006) Heliospheric Physics: Linking the Sun to the Magnetosphere. Sp. Sci. Rev.124:77-90, DOI 10.1007/s11214-006-9130-x

Zurbuchen TH (2007) A New View of the Coupling of the Sun and the Heliosphere. Ann Rev Ast Astrophys 45:297-338, DOI 10.1146/annurev.astro.45.010807.154030

Zurbuchen TH, Fisk LA, Gloeckler G, von Steiger R (2002) The solar wind composition throughout the solar cycle: A continuum of dynamic states. Geophys. Res. Lett.29:1352, DOI 10.1029/2001GL013946

Zurbuchen TH, von Steiger R, Gruesbeck J, Landi E, Lepri ST, Zhao L, Hansteen V (2012) Sources of Solar Wind at Solar Minimum: Constraints from Composition Data. Sp. Sci. Rev.172:41-55, DOI 10.1007/s11214-012-9881-5 